GABY SCHELLEKENS

Language Abstraction in Word of Mouth

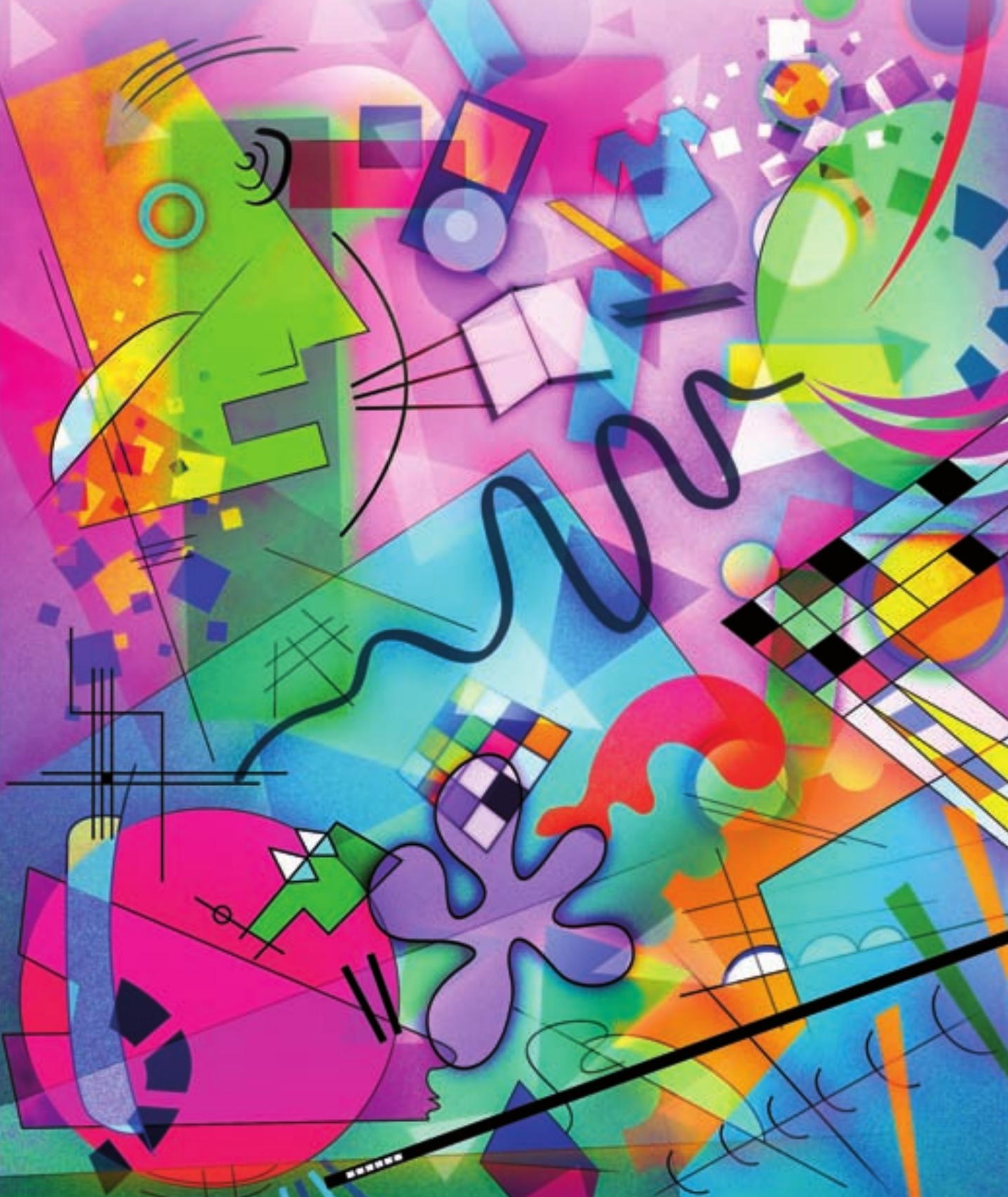




\section{Language Abstraction in Word of Mouth}

Taalabstractie in Mond-tot-mond Communicatie 



\title{
Language Abstraction in Word of Mouth
}

Taalabstractie in mond-tot-mond communicatie

\author{
Proefschrift \\ ter verkrijging van de graad van doctor aan de \\ Erasmus Universiteit Rotterdam \\ op gezag van de \\ rector magnificus \\ Prof.dr. H.G. Schmidt \\ en volgens besluit van het College voor Promoties.
}

De openbare verdediging zal plaatsvinden op

Dinsdag 30 november 2010 om 13.30 uur

door

Gaby Anna Catharina Schellekens

geboren te

's-Hertogenbosch

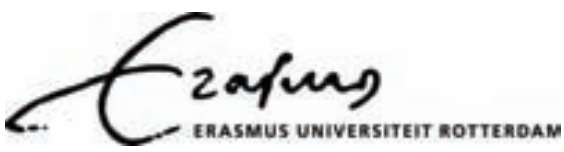


Promotiecommissie

Promotor:

Prof.dr.ir. A. Smidts

Overige leden:

Dr. D. Smeesters

Prof.dr. S. M. J. van Osselaer

Prof.dr. D. H. J. Wigboldus

Copromotor:

Dr.ir. P. W. J. Verlegh

Erasmus Research Institute of Management - ERIM

Rotterdam School of Management (RSM)

Erasmus School of Economics (ESE)

Erasmus University Rotterdam

Internet: http:/ / www.erim.eur.nl

ERIM Electronic Series Portal: http://hdl.handle.net/1765/1

ERIM PhD Series in Research in Management, 218

ERIM reference number: EPS-2010-218-MKT

ISBN 978-90-5892-252-6

(C) 2010, Schellekens

Voorkant: Sjoerd Zaalberg van Zelst www.instorecommercial.nl

Design: B\&T Ontwerp en advies www.b-en-t.nl

Print: Haveka www.haveka.nl

All rights reserved. No part of this publication may be reproduced or transmitted in any form or by any means electronic or mechanical, including photocopying, recording, or by any information storage and retrieval system, without permission in writing from the author. 


\section{Table of Contents}

Chapter 1. Introducing Language Abstraction in Word of Mouth...................... 1

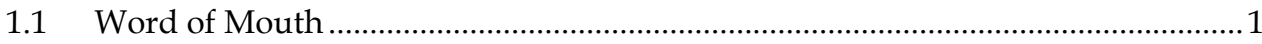

1.2 Language Use in Word of Mouth....................................................................

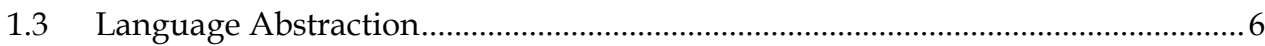

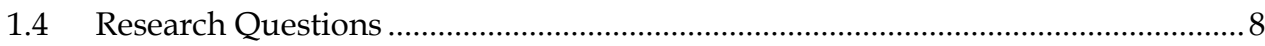

1.4.1 Effect of Senders' Product Attitudes on Language Abstraction

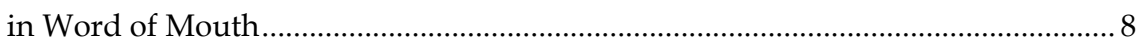

1.4.2 Variations in Language Abstraction by Sender-Receiver Dyad .............................. 10

1.4.3 Effect of Language Abstraction on Receiver .............................................................. 11

\section{Chapter 2. Effect of Sender's Product Attitudes on Language Abstraction}

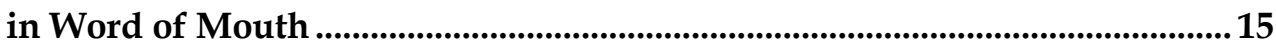

2.1 Systematic Variations in Language Abstraction ......................................................15

2.2 Is Valence or Abstraction Driving Systematic Language Use? ..............................18

2.3 Mechanism of Systematic Use of Language Abstraction .........................................19

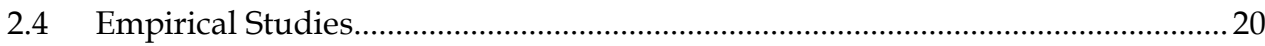

Study 2.1: Language Abstraction in Negative Word of Mouth ................................... 20

Study 2.2: Language Abstraction and Valence........................................................... 25

Study 2.3: Language Abstraction in Word of Mouth ………………………………..... 32

Study 2.4: Language Abstraction and Existing Brand Attitudes ................................. 36

Study 2.5: Language Abstraction in Open Product Descriptions ............................... 40

Study 2.6: Attribution Underlying the Systematic Use

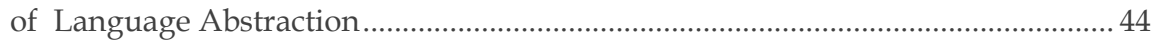

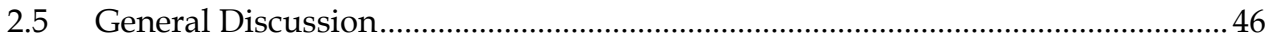

Chapter 3. Language Variation in Sender-Receiver Dyad..................................49

3.1 Strategic Use of Language Abstraction ................................................................... 49

3.2 Effect of Product Attitude of Receiver on Language Abstraction .........................50

Study 3.1: Receiver Induced Systematic Language Abstraction..................................52

3.3 Effect of Communication Goal on Language Abstraction ....................................57

Study 3.2: Effect of Desired Product Attitude on Language Abstraction..................58

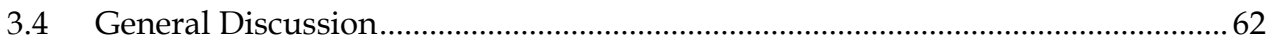


4.1 How does Language Abstraction Affect Receivers?.............................................67

4.2 Effect of Language Abstraction on Inference of Product Attitude of Sender...... 68

Study 4.1: Effect of Language Abstraction on Inferred Product Attitudes

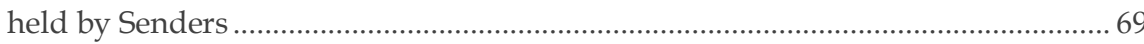

4.3 Effect of Language Abstraction on Behavioral Intention..................................... 74

Study 4.2: Effects of Language Abstraction on Buying Intention ............................. 75

Study 4.3: Effects of Language Abstraction on Buying Intention and Attitude:

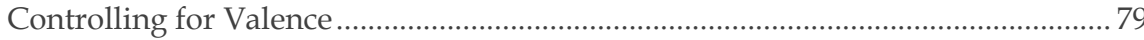

4.4 Mindset Moderates the Persuasiveness of Language Abstraction ....................... 80

Study 4.4: Effect of Receiver's Mindset on Effectiveness

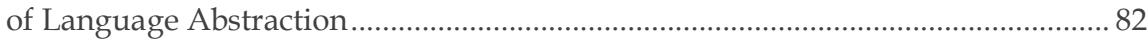

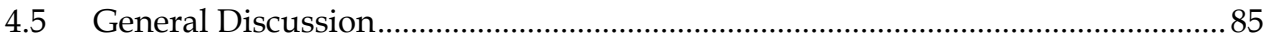

Chapter 5. General Discussion ..................................................................................... 89

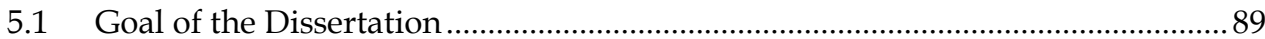

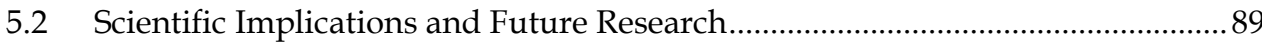

5.2.1 Effect of Senders' Product Attitudes on Language Abstraction

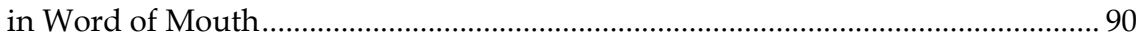

5.2.2 Language Variation in Sender-Receiver Dyad ...................................................... 92

5.2.3 Effects of Language Abstraction on Receivers.......................................................... 94

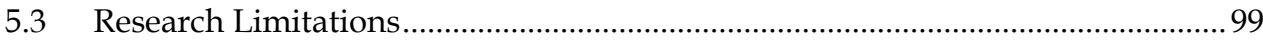

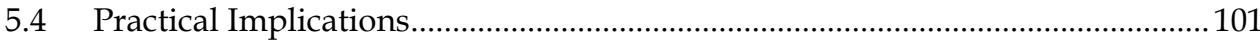

5.4.1 Practical Implications for Word of Mouth Marketing …......................................... 101

5.4.2 Practical Implications for Marketing Communication ........................................... 103

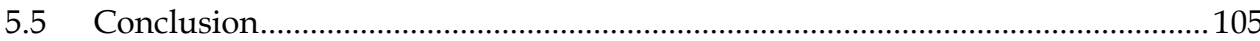

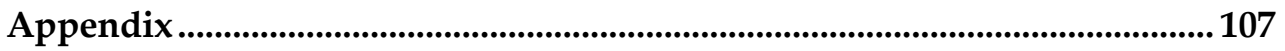

Appendix A. Statements used in Chapter 2 ............................................................. 107

Appendix B. Statements used in Chapter 3 .................................................................. 109

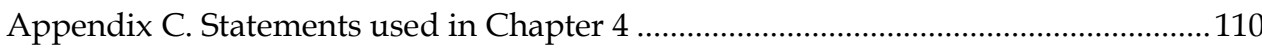

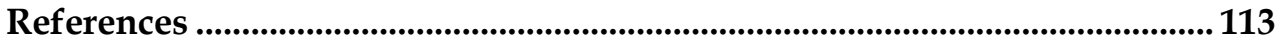

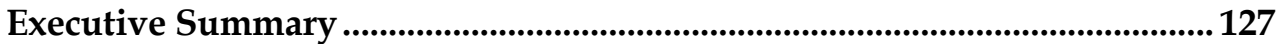




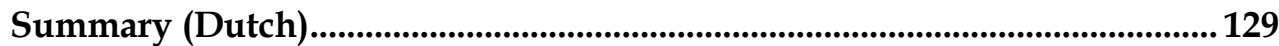

Effect van de Product Attitude van de Zender op Taalabstractie

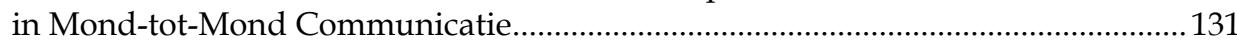

Variatie in Taalabstractie door Communicatiesituatie ............................................ 133

Effecten van Taalabstractie op Ontvangers ……......................................................... 135

Mond-tot-mond Communicatie en Marketingcommunicatie ................................... 137

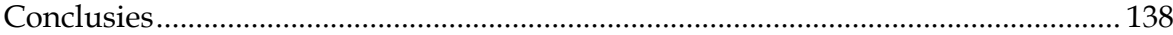

Acknowledgements (Dutch) ................................................................................139

About the author ....................................................................................................... 143

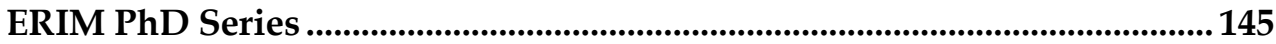





\section{List of Tables}

Table 1.

Study 4.2: Effect of word of mouth (positive vs. negative) and Language Abstraction on Buying Intentions: Mean (SD). 



\section{List of Figures}

Figure 1.

Study 2.2. Effect of product attitude and set of descriptions (positively and negatively correlated descriptions between abstraction and negativity) on the Use of Language Abstraction: Mean (SE)

Figure 2.

Study 2.2. Effect of product attitude and set of descriptions (positively and negatively correlated descriptions between abstraction and negativity) on the Abstraction Index: Mean (SE)

Figure 3. 33

Study 2.3. Screenshots of film clips of positive and negative experiences with product yoghurt.

Figure 4. .34

Study 2.3. Effect of product attitude and product experience on the Use of Language Abstraction: Mean (SE).

Figure 5. 35

Study 2.3. Effect of product attitude and product experience on the Abstraction Index: Mean (SE).

Figure 6a. 37

Study 2.4. Effect of existing attitude and experience with MP3 player on the Use of Language Abstraction: Mean (SE).

Figure 6b. 38

Study 2.4. Effect of existing attitude and experience with T-shirt on the Use of Language Abstraction: Mean (SE).

Figure 7a. 39

Study 2.4. Effect of existing attitude and experience with MP3 player on the Abstraction Index: Mean (SE).

Figure $7 \mathbf{b}$. .39

Study 2.4. Effect of existing attitude and experience with T-shirt on the Abstraction Index: Mean (SE).

Figure 8. Study 2.5 Positive and negative experience with a T-shirt. 41

Figure 9.

Study 2.5. Effect of product attitude and product experience on the Use of Language Abstraction of open response word of mouth: Mean (SE). 
Figure 10a.

Study 3.1. Effect of product experience and receiver product attitude on the Use of Language Abstraction for razor blade: Mean (SE)

Figure 10b. 54

Study 3.1. Effect of product experience and receiver product attitude on the Use of Language Abstraction for yoghurt: Mean (SE)

Figure 11a. 55

Study 3.1. Effect of product experience and receiver product attitude on the Abstraction Index for razor blade: Mean (SE).

Figure 11b. 56

Study 3.1. Effect of product experience and receiver product attitude on the Abstraction Index for yoghurt: Mean (SE).

Figure 12a. 60

Study 3.2. Effect of communication goal and product experience on the Use of Language Abstraction for telecommunication network: Mean (SE).

Figure 12b. 60

Study 3.2. Effect of communication goal and product experience on the Use of Language Abstraction for dish washer: Mean (SE).

Figure 13a. 61

Study 3.2. Effect of communication goal and product experience on the Abstraction Index for telecommunication network: Mean (SE).

Figure 13b.

Study 3.2. Effect of communication goal and product experience on the Abstraction Index for dish washer: Mean (SE).

Figure 14. .73

Study 4.1. Effect of word of mouth (positive vs. negative) and language abstraction (from concrete to abstract: DAV, IAV, SV, Adj) on inferred product attitude of sender: Mean (SE)

Figure 15. .77

Study 4.2. Effect of word of mouth (positive vs. negative) and language abstraction on buying intention (from concrete to abstract: DAV, IAV, SV, Adj): Mean (SE)

Figure 16. 84

Study 4.4. The interaction effect of mindset and language abstraction on product attitude. 
Figure 17.

85

Study 4.4. The interaction effect of mindset and language abstraction on product attitude. 



\section{Chapter 1. Introducing Language Abstraction in Word of Mouth}

"The evidence is abundantly clear: word of mouth is the most important and effective communications channel. Now, the search is for strong, quantifiable research to help marketers navigate this new terrain, where the control rests with the consumer and not the marketer." Ed Keller (2007)

Consumers like to share their experiences with products and services through word of mouth. According to Keller (2007), consumers engage in 120 word-of-mouth conversations per week. It is one of the oldest and most important methods to spread product and service information, and word of mouth has received continuous attention from scientists since the seminal work of Katz and Lazarsfeld's (1955) over five decades ago (Arndt, 1967; Brown \& Reingen, 1987; Chevalier \& Mayzlin, 2006; Frenzen \& Nakamoto, 1993; Watts \& Dodds, 2007).

There are countless ways in which consumers can share positive and negative experiences and evaluations of services and products. For example, when a bright new shirt has lost its color after washing it once or twice, one could say to a friend "My brand X shirt has faded," or one could say "My brand X shirt was of poor quality". Where the former is a very concrete description of what has happened, the latter generalizes this experience to the overall quality of the shirt by using more abstract wording. But when and why does a consumer use which version? And what is the effect of each version on the listening party? The broad purpose of this dissertation is to improve our understanding of language use in word of mouth and its effects on receivers.

\subsection{Word of Mouth}

Word of mouth is commonly defined as (1) interpersonal communication (2) about products, services or company related issues (3) in which none of the participants are marketing sources (Arndt, 1967; Bone, 1995; Westbrook, 1987). Interpersonal 
communication means that no mass media is used, but it can involve blogs and internet forums. Traditionally word of mouth is non commercial. However, companies recently started using various strategies (e.g., financial rewards) to increase the likelihood that people will talk about their specific products (word-of-mouth marketing; Godes \& Mayzlin, 2004; Ryu \& Feick, 2007; Tuk, et al., 2009). Consumers often ask other consumers for advice when considering a new service or product (Arndt, 1967; Brown \& Reingen, 1987). People are interested in and attend to word-ofmouth referrals, because they are perceived as credible and custom-tailored (Wirtz \& Chew, 2002). As the credibility of the traditional marketing is declining, the power of word of mouth is thought to increase (Keller, 2007). The next paragraphs will present a brief overview of previous research on word of mouth.

A lot of research on word of mouth focused on source characteristics (Brown \& Reingen, 1987; Herr, Kardes \& Kim, 1991; Price, Feick, Higie, 1989). Overall, consumers prefer information from experts (Brown, Broderick \& Lee, 2007; Feick \& Price, 1987; Klucharev, Smidts, \& Fernandez, 2008), and from people who are perceived to be similar to themselves (Gilly, Graham, Wolfinbarger, \& Yale, 1998). Another well studied element of word of mouth is the relationship between the sender and the receiver, more precisely the tie strength (Brown \& Reingen, 1987; De Bruyn \& Lilien, 2008; Godes \& Mayzlin, 2004; Van Hove \& Lievens, 2009). Family members and friends are considered to be 'strong ties' and acquaintances are labeled 'weak ties'. Word of mouth from strong ties has been shown to be perceived as more credible (Van Hove \& Lievens, 2007), and to have a greater influence on receiver behavior (Bansal \& Voyer, 2000; De Bruyn \& Lilien, 2008). Additionally, the spread of (online) word of mouth has received increasing attention from researchers (e.g., Goldenberg, Libai, Moldovan, \& Muller, 2007; Watts \& Dodds, 2007). For instance, Van der Lans et al. (2009) developed a viral branching model which predicts how many customers will be reached through electronic word of mouth in a viral marketing campaign.

In word of mouth, a variety of motivations may influence what consumers say to one another (Dichter, 1966; Mangold, Miller, \& Brockway, 1999; Sundaram, Mitra, \& Webster, 1998; Thurau, Gwinner, Walsh, \& Gremler, 2004). The satisfaction with a product or service is an important driver of word of mouth (Matos \& Rossi, 2008; Brown, et al., 2005). Customer satisfaction or dissatisfaction results from a product experience being congruent or incongruent with consumers' expectations (e.g., Oliver, 1997). When consumers are very satisfied or dissatisfied, they are more likely to spread word of mouth (Oliver, 1997). While satisfaction has a stronger relationship with 
positive word of mouth than loyalty, disloyalty has a stronger relationship with negative word of mouth than dissatisfaction (Matos \& Rossi, 2008).

Next to product or service (dis)satisfaction, word of mouth can also be driven by self or other focused motives (Sundaram, Mitra, \& Webster, 1998; Thurau, Gwinner, Walsh, \& Gremler, 2004). For self focused motives, consumers can share word of mouth to self enhance (e.g., come across as an expert), to relieve or regulate negative emotions (e.g., reduce anxiety), and to gain vengeance (e.g., revenge against company). Other self focused reasons to engage in word of mouth are high product involvement or when seeking advice is also self focused. Additionally, one can engage in word of mouth because of other-related motives such as altruism, which can be helping another consumer with useful advice or helping the company.

What consumers are actually saying in offline and online word of mouth has mainly been examined by measuring the amount and the valence of the product and service messages (Chevalier \& Mayzlin, 2006; East, Hammond \& Wright, 2007; Frenzen \& Nakamoto, 1993; Godes \& Mazlin, 2004; Smith \& Vogt, 1995). For instance, the amount of word of mouth about a movie offers significant explanatory power for box office revenues and performance (Duan, Gu, \& Whinston, 2008; Liu, 2006). The popularity of studying the amount and valence of word of mouth stems in part from the ease with which these variables can be measured in (online) markets. Other aspects are more difficult to measure, and therefore less popular to use.

Where earlier research mainly focused on the effect of the amount of word of mouth on receivers, the valence of the word of mouth has been receiving more attention lately (Matos \& Rossi, 2008). It is argued that extremely positive and extremely negative word of mouth are the most common examples of word-of-mouth communication (Chevalier \& Mayzlin, 2006; Duan, Gu, \& Whinston, 2008; Mazzarol, Sweeney, \& Soutar, 2007). Whereas positive word of mouth has been shown to be more prevalent (East, Hammond \& Wright, 2007), negative word of mouth seems to be more influential (Bone, 1995; Laczniak, DeCarlo, \& Ramaswami, 2001), although East and colleagues (2008) have shown that this needs not always be true.

In sum, previous research on word of mouth has created an impressive body of knowledge about the valence and amount of word of mouth, how it spreads, and how it is influenced by the characteristics and motives of the source and receiver of word of mouth, while there has been very little empirical research on the content of word of mouth (i.e., what is being said and how). 


\subsection{Language Use in Word of Mouth}

Through language people create and exchange meaning, and maintain social relationships (Fiedler, 2008a; Guerin, 2003; Holtgraves \& Kashima, 2008; Semin, 2000). Innumerable research possibilities lay in the semantics ('what words refer to'), syntactics ('rules and consequences of words together'), and the pragmatics ('interpretation of words in social context') of language. Previous research has for instance examined the effect of language framing on persuasion, such as the effects of direct versus indirect product claims (Kardes, Kim, \& Lim, 1994), and a loss frame ('don't miss out on ...') compared to a gain frame ('enjoy ...')(Lee \& Aaker, 2004; Maheswaran \& Meyers-Levy, 1990). Language also encompasses a variety of (para)linguistic phenomena which can affect receivers, such as features of utterances as interjection, intonation, tag questions, and hesitations (e.g., 'uh', 'hmm')(Blankenship \& Holtgraves, 2005; Brennan \& Williams, 1995). This stresses that 'how' something is said may at times be just as important as 'what' is being said (Blankenship \& Holtgraves, 2005; Fiedler, 2008a; Fitzsimons \& Kay, 2004). Despite the obvious importance of this subject, language seems not to be receiving the attention that it deserves (Holtgraves \& Kashima, 2008; Semin \& Fiedler, 1988; Semin, 2000; Higgins, 1981). Especially, since language is an essential aspect of word of mouth, it is surprising that an in depth understanding of language use in word of mouth was still lacking. But how do we decide upon which dimension of language to focus? What linguistic aspect would be most likely to improve our understanding of word of mouth?

A main element of word of mouth is the interpersonal aspect: a consumer shares a service or product experience with another consumer. No two word-of-mouth messages are the same. Part of word-of-mouth's strength lies in the option to tailor each message to a specific situation or audience. In other words, the context of the social interaction affects which product information is being shared and how this is communicated. Previous research in social psychology revealed that elements in the interpersonal communication context can affect the abstractness of the language that is used in communication about human behavior (Douglas \& Sutton, 2003; Maass, Salvi, Arcuri, \& Semin, 1989; Maass, Milesi, Zabbini, \& Stahlberg, 1995; Von Hippel, Sekaquaptewa, \& Vargas, 1997; Webster, Kruglanski, \& Dwight 1997; Wigboldus, Spears, \& Semin, 2000).

Language abstraction is an essential property of language. The abstractness of language can vary from concrete ('he hit someone') to abstract ('he is aggressive'; 
Semin \& Fiedler, 1988). The use of more abstract language implicates a belief that the event reflects an underlying disposition or trait of the actor. The exact nature of language abstraction will be explained in the next paragraph 1.3. In general, language abstraction enables the same intention to be communicated in a variety of different ways, and these choices are a reflection of the intended message of a sender (Semin \& Fiedler, 1988, 1989, 1991). Subsequently, the abstractness of a description can be used to infer information about the sender of the message; it can reveal information about the psychological and social processes underlying message production (Maass, et al., 1995; Semin \& Fiedler, 1988). Additionally, language abstraction could also play an important role in message perception and its effect on receivers' cognitive inferences (Semin, 2000; Holtgrave \& Kashima, 2008; Wigboldus, et al., 2000). Independently of the content of the message, language abstraction can convey meaning to a receiver, because it directs the attention of a receiver to a specific facet of the intended message and shapes the cognitive processes of the receiver. Thus, the abstractness of language conveys one's interpretation and evaluation of information, and hereby serves a subtle function in communication.

Substantial literature has shown that people systematically vary language abstraction in interpersonal communication (for overviews see Wigboldus \& Douglas, 2008 and Fiedler, 2008b). The ins and outs of this systematic use of language abstraction will be discussed thoroughly in paragraph 1.4.1 and 2.1. In general, more abstract descriptions are used for stereotype consistent behavior and more concrete descriptions for stereotype inconsistent behavior. In communication of stereotypes, the linguistic mechanism that underlies the biased language use revealed a vicious circle of stereotype transmission and maintenance.

The objective of this dissertation is to examine consumers use of language abstraction in word of mouth, and the effects of language abstraction on receivers. More specifically, I first sought to establish how and when consumers systematically frame product related message more concretely and abstractly. Previous studies on language abstraction were limited to the interpersonal domain. This dissertation is the first to study language abstraction in the descriptions of experiences with inanimate objects, products in my case. Additionally, this dissertation will study the impact of elements in the sender-receiver dyad on the language use of the sender, such as the receiver's product attitude and a sender's communication goal. Moreover, the impact of language abstraction on receivers will be studied. I propose that above and beyond the actual content and valence of the message, the language abstraction of a message 
may reveal information about the sender, such as the attitude of the sender about the object of communication, and the motive for the communication. Furthermore, this dissertation will show that consumers receiving word of mouth can pick up on subtle cues provided by the sending consumer's linguistic patterns, and how they are affected by the language abstraction of word of mouth. Finally, it introduces a moderator in the persuasiveness of language abstraction. In the next section language abstraction according to the linguistic category model is introduced, and the three core research questions of this dissertation are established.

\subsection{Language Abstraction}

The linguistic category model (Semin \& Fiedler, 1988) provides a framework for studying the language that people use to describe social events. It focuses on the causes and consequences of using abstract versus concrete descriptions to describe events and experiences. This framework has been used to study language use in a variety of settings and has been successfully applied in various languages, including English, Italian, Greek, Japanese, German, and Dutch (Maass et al. 1995, 1998; Maass, Karasawa, Politi, \& Suga, 2006; Webster, Kruglanski, \& Dwight 1997; Schmid \& Fiedler, 1998; Stapel \& Semin, 2007). This framework will be used to study language abstraction in communication about products and services.

To systematically study language abstraction, the linguistic category model refrains from a subjective analysis of the content and meaning of specific words, but focuses on more general linguistic aspects of language use (Semin \& Fielder, 1988). In particular, the model focuses on the level of abstractness of the verbs and predicates that are used to describe behavior. The model distinguishes four different categories of verbs and predicates, which are presented below, in order of increasing levels of abstractness. At the most concrete level are Descriptive Action Verbs (DAV), which convey non-interpretative descriptions of single, observable events (e.g., "Kirk hits Matt"). At the second level, Interpretive Action Verbs (IAV) refer to a more general class of specific observable behaviors. IAVs do not preserve the perceptual features of an action (e.g., "Kirk hurts Matt"). State Verbs (SV) form the third category. They describe an enduring unobservable (mental) state of the actor, and not a specific behavior (e.g., "Kirk hates Matt"). The most abstract category consists of Adjectives (Adj), which describe only the sentence subject and generalize the behavior to the trait level, which is presumably stable across events (e.g., "Kirk is aggressive"). 
A single event can be described at different levels of abstraction. For example, a person who sees Kirk hitting Matt may use the concrete statement "Kirk hits Matt," which describes Kirks behavior in this specific situation. The statement is easy to verify and leaves little room for dispute. It is likely to be used by people who feel that this behavior is due to factors outside Kirk, and who do not see Kirk as a violent person in general. On the other hand, someone who is observing this situation could also choose to say "Kirk is aggressive," which is more informative about Kirk's personality and suggests that Kirk acts violently in many situations and toward many others. This statement is more difficult to verify and more open for dispute, since it generalizes this one behavior to the level of Kirk's personality. When a behavior is described in more abstract language, people perceive this behavior to be more stable, and more likely to be repeated by this person. Thus, with increasing abstraction, descriptions become more informative about the actor in the event, and less informative about the situational circumstances (Semin \& Fiedler, 1988; Wigboldus, et al., 2000).

Note that concrete and abstract language according to the linguistic category model are not comparable to how the concepts 'concrete' and 'abstract' are examined in previous marketing research. Differences in persuasiveness of concrete and abstract information are well documented in marketing (Aggarwal \& Law, 2005; Corfman, 1991; Hamilton \& Thompson, 2007; Keller \& Block, 1997; Kim \& Meyers-Levy, 2007; Kim, Rao, \& Lee, 2007; Lee \& Ariely, 2006; Lee, Keller \& Sternthal, 2010; Mackenzie, 1986; Maheswaran \& Sternthal, 1990; Meyers-Levy \& Zhu, 2007; Zhao, Hoeffler \& Zauberman, 2007). In one of the first papers on concrete and abstract information, Mackenzie (1986) showed that concretely worded messages attract more attention and are more persuasive than abstractly worded messages. Recent research focused on specific situations in which abstract information is more persuasive, such as the (interaction)effects of abstractness, consumer processing and cognitive resources on context effects (Kim \& Meyers-Levy, 2007). In these marketing studies, concrete- and abstractness is manipulated in line with the meaning of the concepts in everyday language: abstract information is imprecise and subjective (e.g., overall quality), and concrete information is detailed and objective, and often in addition to abstract information (e.g., price, ranking with stars). For instance, an abstract message was 'according to industry sources, many watches breakdown due to water getting into the case', while in the concrete version the word 'many' was replaced with '3 out of every 4' (Mackenzie, 1986). The main difference between previous marketing research on concrete- and abstractness and language abstraction according to the linguistic 
category model lays in the fact that in the latter version language abstraction - the level of abstractness - is established on the use of verbs and predicates, not on the addition of facts and specifics. Language abstraction evolves around speaking more concrete or abstractly, not solely about meaning something more concrete or abstractly. Adding facts or detailed information may change the concrete or abstractness of information, but this does not change the language abstraction of the information according to the category model. Thus, both descriptions with 'many' and ' 3 out of 4 ' would be rated as Interpretive Action Verbs descriptions, based on the use of the verb 'breakdown'.

\subsection{Research Questions}

\subsubsection{Effect of Senders' Product Attitudes on Language Abstraction in Word of Mouth}

Research in social psychology (Maass et al., 1995; Wigboldus et al., 2000) has shown that the level of abstraction that people use to describe an event is influenced by their a-priori expectations about the actor in the event. More specifically, behavior that is congruent with one's expectations about the actor will be described in a more abstract manner, because such language emphasizes the fact that the behavior is thought to represent a stable and typical behavioral tendency of this person. On the other hand, if an actor behaves in a way that is unexpected and surprising, people will describe this behavior concretely, which dissociates the behavior from the actor and frames it as a one time event (Wigboldus et al. 2000). These expectations can be based on many grounds, including for example on group membership or stereotypes (Maass et al., 1989, 1995, 1998; Wigboldus et al., 2000). For example, Maass et al. (1989) asked participants to describe the behavior of a character in a cartoon. If the behaviors shown in the cartoons were positive (e.g., helping someone), the participants used more abstract language when the characters were ingroup members (e.g., students from the same university, or people from the same city as the participants) than when the characters were outgroup members. This finding was reversed for negative behaviors such as littering, where descriptions of negative behavior were more abstract for outgroup members than for ingroup members. This phenomenon is referred to as the linguistic intergroup bias. Wigboldus et al. (2000) examined whether the same effect occurs when people describe characters who engage in behaviors that are congruent or incongruent with the observer's stereotype of the actor (regardless of whether this 
actor is an ingroup or outgroup member). Indeed, their findings showed a linguistic expectancy bias: people use more abstract language to describe behavior that is congruent with their stereotype-based expectations of the actor.

This dissertation first sought to establish if consumers systematically use language abstraction when sharing product experiences with other consumers ${ }^{1}$. The first research question is:

Do consumers systematically use language abstraction in word of mouth, and what is driving this behavior?

I propose that the level of abstraction that people use to describe a product experience is influenced by their a-priori attitudes about the product in the experience. Chapter 2 contains six studies in which a variety of manipulations for the independent and dependent variables are used, and a wide range of product categories (ranging from durables to fast moving consumer goods) are studied to demonstrate the robustness of a systematic variation in consumers use of more concrete and abstract word-of-mouth messages. More specifically, this chapter shows that product experiences that are congruent with consumers' brand attitudes are communicated more abstractly than experiences that are incongruent with brand attitude.

A potential criticism of previous research on linguistic bias lies in the inherent relationship between the abstractness of a description and its valence. One could argue that a more abstract description of negative word of mouth paints a less favorable picture of the brand, because abstract language portrays an event as being more characteristic of the brand. For instance, the abstract version of a negative product experience "My brand X shirt was of poor quality" can be considered as a more negative description than the more concrete version "My brand X shirt has faded". In that case it may be that systematic use of language abstraction is not driven by the abstractness of language, but by the valence inherent to the levels of the linguistic category model. Although some studies have attempted to address this alternative explanation (Maass, et al., 1989; Douglas \& Sutton, 2003), there is no consistent empirical evidence that excludes valence as an alternative hypothesis for the

\footnotetext{
${ }^{1}$ For sake of readability we will use the terms product experiences and product descriptions in this dissertation, but we have examined word of mouth about both products and services. See e.g., Appendix A for the stimulus material on the service communication network, or Appendix $\mathrm{C}$ for the descriptions of the service of a mortgage broker.
} 
systematic use of language abstraction. This dissertation will demonstrate that variations in the valence of language cannot account for the systematic use of language abstraction, and the effects of language abstraction on consumer behaviour.

Furthermore, it will be explored what is driving this systematic language use in word of mouth. This dissertation will show that the variations in language abstraction are driven by the fact that consumer's inferences about the cause of an experience are determined by their a priori expectations. Hence, the first empirical chapter (Chapter 2) will answer the first research question by investigating the sender's use of language abstraction in word of mouth, demonstrating the underlying mechanism and excluding an important alternative explanation for the phenomenon.

\subsubsection{Variations in Language Abstraction by Sender-Receiver Dyad}

During word of mouth, consumers' language use may not only be affected by the product experience and product attitude of the sending consumer, but also by other variables, such as the goal with which consumers communicate or various characteristics of the receiver. Several studies have shown that the systematic variations in language abstraction escape conscious access and are difficult to control (Maass et al., 1995; Von Hippel, Sekaquaptewa, \& Vargas, 1997; Webster, Kruglanski, \& Pattison, 1997). People generally seem unaware of the abstraction level at which they describe an event, and their motivations for choosing a certain level do not seem to be cognitively accessible (see also Franco \& Maass, 1996, 1999). In spite of this, there are a number of studies that show that language abstraction may be used strategically, corresponding for example to the motivation to protect one's ingroup (Maass et al., 1996, 1998; Sekaquaptewa et al., 2003; Schmid \& Fiedler, 1998), to reach cognitive closure (Webster, Kruglanski, \& Pattison, 1997), or in response to communication instructions (Douglas \& Sutton, 2003; Douglas, Sutton, \& Wilkin, 2008; Semin, et al., 2005; Rubini \& Sigall, 2002; Wenneker, et al., 2005).

These findings suggest that the language that consumers use in word of mouth may not only be driven by their a priori attitudes about product experiences, but also by other elements of the sender-receiver dyad. I argue that language abstraction can be used adaptively to adjust to the word-of-mouth sender-receiver dyad, including such elements as the underlying goal of a product referral or characteristics of a conversation partner. 
Thus, the second research question is:

Do consumers adapt the abstractness of their language to the goal of the communication and the attitude of a receiver?

This dissertation will show that a sender's use of language abstraction is affected by the characteristics of a receiving consumer. More specifically, more abstract language is used for product information that is consistent with the product attitude of a receiver than for product information that is inconsistent with the receiver's attitude. I label this phenomenon the Receiver Induced Systematic Language Abstraction. Moreover, it is hypothesized that the language abstraction of a product message will also be affected by the goal of the product referral. Consumers will be shown to vary language abstraction based on a frequent communication goal in marketing, i.e. to persuade another consumer of the superiority of a product. Thus, Chapter 3 reveals the sender's adaptive use of language abstraction triggered by two crucial elements in a senderreceiver dyad.

\subsubsection{Effect of Language Abstraction on Receiver}

In addition to studying the language that is used by consumers sending word of mouth, this dissertation will examine the effects of language abstraction on consumers receiving word of mouth. Research on language abstraction has mainly focused on the factors that cause people to describe events in abstract or concrete terms. Far less is known about the ways in which receivers respond to communicators' use of abstract versus concrete language. Some studies showed that the level of language abstraction that is used in descriptions of events influences the extent to which these descriptions affect the readers of these descriptions. Wigboldus and colleagues (2000) for example demonstrated that stories which described behavior of a stereotyped actor led to more dispositional inferences about the actor when this behavior was consistent with the observer's stereotypes of the actor. Importantly, this effect was mediated by the level of abstraction of the language that was used to describe this behavior. This finding is in line with the notion that abstract descriptions are less focused on the specific circumstances under which the behavior was performed, and are more informative of the actor (Semin \& Fiedler, 1988; Wigboldus, Semin, \& Spears, 2006). 
Generally speaking are receivers more positive about a product or service after receiving positive word of mouth, and more negative after receiving negative word of mouth (Bone, 1995; Chevalier \& Mayzlin, 2006; East, Hammond, \& Wright, 2007). But it is not clear how the valence of the word of mouth and its language abstraction interact. Is positive word of mouth more persuasive when it is phrased abstractly or concretely? And does abstractness have the same impact with negative messages? On the one hand, the linguistic category model (Semin \& Fiedler, 1988) postulates that abstract descriptions relate an event or behavior to dispositional rather than situational factors, and generalize the information to the level of the actor, or, in the present context, to the level of the product. Framing product information as a stable quality could have a stronger impact than framing it context dependently in a concrete word-of-mouth message. On the other hand, abstract descriptions are more open to dispute and less verifiable, while concrete descriptions are often more vivid, and vividness has been shown to have a persuasive effect (Kim, Kardes, \& Herr, 1991; Kisielius \& Sternthal, 1984). The third research question is:

How does language abstraction moderate the impact of word of mouth on receivers, and what moderates this effect?

Chapter 4 will first demonstrate that the language abstraction of a word-of-mouth message can be used to infer a sender's product attitude. More specifically, senders are inferred to have a more positive attitude when communicating a more abstract (vs. concrete) positive product referral, and when communicating a more concrete (vs. abstract) negative referral. In addition, the chapter will show that receivers have a higher buying intention after more abstract (vs. concrete) positive word of mouth and after more concrete (vs. abstract) negative word of mouth. Finally, chapter 4 will show how the objective of the receiver moderates the persuasiveness of language abstraction. Information that is consistent with a receiver's focus leads to a higher buying intention than inconsistent information.

Hence, the findings of Chapter 3 suggest that senders take the effects of language abstraction on receivers into account when describing product experiences, and in Chapter 4 will examine the actual effects of the abstractness of a word-of-mouth message on receivers. Chapter 2 to 4 complete the communication cycle from the sender's use of language abstraction to the impact of abstractness on receivers. In 
chapter 5, I will elaborate on the scientific and practical implications of the findings of this dissertation, and discuss suggestions for future research. 



\section{Chapter 2. Effect of Sender's Product Attitudes on Language Abstraction in Word of Mouth}

\subsection{Systematic Variations in Language Abstraction}

Research in social psychology has shown that the abstractness of language that people use to describe another person's behavior is influenced by the extent to which this behavior is congruent or incongruent with their expectations (Maass, Milesi, Zabbini, \& Stahlberg, 1995; Maass, Salvi, Arcuri, \& Semin, 1989; Schmid, \& Fiedler, 1998; Wigboldus, Spears, \& Semin, 2000). These expectations can be based on stereotypes, ingroup or outgroup membership, or general liking or disliking of the person. Maass (1989) first demonstrated this systematic use of language abstraction in descriptions of human behavior, and referred to this phenomenon as the linguistic intergroup bias. For instance, positive behavior of ingroup members and negative behavior of outgroup members is described more abstractly than negative behavior of ingroup members and positive behavior of outgroup members. Additionally, Wigboldus and colleagues (2000) demonstrated that people use more abstract language to describe behavior that is congruent with their (stereotype-based) expectations of an actor, and dubbed this effect the linguistic expectancy bias.

Previous research on language abstraction has been limited to the interpersonal domain. This dissertation will examine language use in the descriptions of experiences with inanimate objects. More specifically, this chapter will examine how consumers' use of abstract versus concrete language in word of mouth is influenced by the congruency or incongruency between their product attitudes and their product experiences.

It is not self-evident that the congruency or incongruency between product attitudes and product experiences should lead to the same kind of variations in the use of language abstraction as has been shown with descriptions of human behavior. In prior research on linguistic bias, expectations about a person's behavior were based on stereotypes or group membership. The level of language abstraction used in descriptions of someone's behavior implicitly communicates a belief about the degree to which this behavior reflects an underlying disposition or trait of the actor (Semin \& 
Fiedler, 1988; Wigboldus et al., 2000). At a more general level, this assumes that people who find themselves in a certain situation can choose to act in a certain way, and that this choice reveals something about their dispositions or traits. Inanimate objects such as products, however, do not choose to behave differently in different situations. A fountain pen is not eager to write a high quality dissertation, and cannot refuse to write low quality work. Products cannot choose to act in a certain way, and are therefore not evaluated on those grounds. This may prevent consumers from varying language abstraction in product descriptions based on their attitudes towards the product. In addition, product experiences by definition are interactions between products and consumers, which is why word of mouth often has the user (which may be either the communicator or another consumer) rather than the product at the center of attention (e.g., 'the guy next door drives a BMW'). In other words, when consumers describe a product experience, they may focus on their own feelings and thoughts, or (if they describe another consumer's experience) they may share the experience in order to communicate something about the user rather than the product. It is not obvious, therefore, that consumers' use of language abstraction in product descriptions should be influenced by their attitudes toward the product.

This dissertation will examine the extent to which language abstraction in consumers' descriptions of product experiences is influenced by their a priori attitudes towards the product. The focus will first be on negative product experiences (Study 2.1 and Study 2.2). Compared to positive experiences, these are often thought to have a larger impact on consumers' preference and satisfaction judgments (Mittal, Ross \& Baldesare, 1998; Mizerski, 1982), and are more likely to lead to word of mouth (e.g, Anderson, 1998; Richins, 1983). Studies 2.3 through 2.6 generalize this to the domain of positive product experiences.

In line with the literature on linguistic biases in interpersonal interactions, I hypothesize that consumers use more abstract language to describe negative experiences with a product of a brand towards which they hold an unfavorable brand attitude. In this case, consumers will more likely view the negative experience as more typical and representative of the brand. In other words, consumers with an unfavorable attitude towards the product are more likely to view its poor performance as the result of an enduring characteristic (flaw), which is likely to occur on various occasions. Conversely, consumers with a favorable brand attitude will be more likely to regard a negative experience as an exception to the rule and be reluctant to 
generalize the experience to the brand level. They would choose to describe the experience in concrete terms, which frames the experience as a one time event.

H1a Negative product experiences will be communicated more abstractly by consumers with an unfavorable (vs. a favorable) product attitude.

For positive experiences, I expect the pattern to be reversed. More specifically, I expect that consumers with a favorable brand attitude will be more likely to regard positive experiences as typical for the brand, because positive experiences are more congruent with their favorable attitude towards it. This should be reflected in the use of abstract language, which generalizes the experience to the brand level.

H1b Positive product experiences will be communicated more concretely by consumers with an unfavorable (vs. a favorable) product attitude.

When tested in combination, Hypotheses $1 \mathrm{a}$ and $1 \mathrm{~b}$ should lead to a cross-over interaction between product experience (positive vs. negative) and brand attitude (favorable vs. unfavorable). With six studies this chapter robustly demonstrate that consumer systematically bias linguistic abstraction in word of mouth. Studies 2.1 and 2.2 focus on negative word of mouth and studies 2.3 to 2.6 examine the use of language abstraction in messages about both positive as negative product experiences. In Study 2.1, participants use a product themselves, and then communicate this experience to another consumer. In Study 2.2 these findings are replicated with an experience that another customer has with a product. Study 2.3 studies language use with both positive and negative experiences, and Study 2.4 replicates this study with existing brand attitudes. Study 2.5 examines systematic language use with an open dependent variable (participants are asked to describe a product experience in their own words). Finally, Study 2.6 examines the mechanism driving language use (see paragraph 2.3 for explanation). 


\subsection{Is Valence or Abstraction Driving Systematic Language Use?}

One potential criticism of research on systematic language use lies in the inherent relationship between the abstractness of a description and the valence of that description. Because abstract language portrays a behavior as being more characteristic of the actor, the use of more abstract descriptions of negative experiences paints a less favorable picture of the actor. It may very well be that consumers who hold an unfavorable attitude toward a brand choose to describe a negative experience in more abstract terms, simply because these terms paint a more negative picture of the brand. Conversely, consumers with a favorable brand attitude would prefer concrete language to describe a negative experience, because this gives the least negative description of the event and thus reflects their favorable opinion. This confound also provides a possible alternative explanation for the linguistic intergroup bias studied in social psychology: if an ingroup member acts undesirably, people may be more likely to describe this behavior in concrete terms (e.g., 'Mary dropped rubbish on the ground') because that description is less negative about the ingroup member than a more abstract description (e.g., 'Mary is dirty'), which generalizes the event to the personality of the actor.

Maass and colleagues (1989) addressed this explanation in their seminal work on linguistic intergroup bias. The desirability of the descriptions used in the first study of this paper was measured in a post-hoc test. The authors then collapsed the lower (DAV and IAV) and higher (SV and Adj) levels of the linguistic category model, and found that desirability increased with abstractness for both positive and negative behaviors. Since this occurred for both behaviors, the differences in desirability could not account for the linguistic intergroup bias that they observed in that study. In a later study, Douglas and Sutton (2003, Study 5) examined the relationship between abstraction and valence by mediation analyses. These authors asked a separate group of participants to rate the valence of the descriptions that were used in this study, and the language abstraction and the valence of the descriptions were found to be related. However, mediation analyses showed that the valence of descriptions did not mediate the relationship between expectations and language abstraction.

I propose that variations in the valence of language do not account for the systematic use of language abstraction and the effects of variations in language abstraction on consumer behaviour. On of the aims of this chapter is to provide consistent empirical evidence to exclude valence as an alternative hypothesis for the systematic use of language abstraction. To examine the role of language valence in 
word of mouth, the valence of the word-of-mouth communication is measured and through mediation it is demonstrated that valence does not mediate the interaction effects of product attitude and product experience on the systematic use of language abstraction (Study 2.3 and 2.5). Additionally, the valence of the study material is kept constant in Study 2.1, and it still revealed confirming results for the systematic language use. More excitingly, Study 2.2 is the first study in the literature in which the relationship between valence and abstraction is manipulated experimentally. The valence of product descriptions is manipulated so that the valence of the descriptions is either positively or negatively correlated with language abstraction. Strikingly, no interaction between language abstraction and language valence is found, which shows that consumers' use of more abstract language to describe product experiences that are congruent with their attitudes does not depend on a positive correlation between valence and abstraction.

\subsection{Mechanism of Systematic Use of Language Abstraction}

Semin and Fiedler's $(1988,1989)$ four-level linguistic model proposes that people vary language abstraction because of its cognitive implications, such as informativeness about the situation, and the temporal stability of the quality expressed in the sentence. Semin and Fiedler $(1988,1989)$ provided evidence for the fact that language abstraction relates to these constructs. From a receivers side, Wigboldus, Semin and Spears (2006) have also shown that receivers infer a more situational or dispositional causation on variations in language abstraction. This dissertation will empirically test the meditational process for a sender's use of more concrete or abstract language. In line with Semin and Fiedler's (1989) theorizing, I propose that these variations in language abstraction are caused by the fact that consumer's inferences about the cause of an experience are determined by their a priori expectations (see Wenneker, Wigboldus, \& Spears, 2005, for a similar argument). More specifically, if a product experience is in line with our attitude towards that product, we tend to generalize our observation to other situations, and regard it as a confirmation of our ideas. Thus, if we a priori consider a person to be aggressive, and we see this person hitting someone, we are likely to make a dispositional inference, and regard this behavior as a manifestation of the underlying disposition. In line with this reasoning, research on action identification showed that describing someone's actions at a high level of action 
identification is associated with claiming that they are acting intentional (Kozak, Marsh, \& Wegner, 2006).

On the other hand, if an observation counters our expectations, we tend to cognitively isolate the behavior from the person, and attribute the behavior to the environment or other actors. Similarly, in the context of product experiences, this would imply that for experiences that are not in line with our attitudes regarding the brand or product, consumers are more likely to seek explanation in the context, which may include the situation in which the product is consumed or the person who is consuming it. Thus, for product experiences which are incongruent to the product attitude, consumers may be better able to generate disabling conditions for the product experience (e.g., dependent on specific environmental circumstance or on an user), which blocks the experience to be seen as a disposition of the brand (Chandon \& Janiszewski, 2009).

In sum, if product experiences are congruent with consumer's a priori product attitudes, consumers will be more likely to infer that the experience can be attributed to the product itself, and not to the user or the usage situation. This inference translates into the use of more abstract language to describe the experience. Along the same lines, incongruent experiences are more likely to be attributed to the user or the usage situation, which favors the use of concrete language to describe the experience. This mechanism represents a central tenet of the Linguistic Category Model, and answers the question: "Why do consumers systematically use language abstraction?" Accordingly, it is hypothesized:

H2 The inferred cause of the experience mediates the effect of product attitude and experience on systematic variations in the use of language abstraction.

\subsection{Empirical Studies}

\section{Study 2.1: Language Abstraction in Negative Word of Mouth}

This study sought to establish a systematic use of language abstraction when consumers describe negative experiences with products. Participants were asked to test a product; they tested a ballpoint pen, and then described their experience with it. 
The pen was invisibly damaged so that its writing quality was rather poor. To manipulate consumers' attitudes toward the pen, they were either told that they were testing a Parker pen (a well-known high quality brand) or that they were testing a BIC pen (an equally well-known low quality brand). After testing the pen on a piece of paper, they were asked to describe their experience to another student. In line with earlier research on language abstraction, participants were presented with a set of four different descriptions that they could use to describe their experience. These four descriptions correspond to the four different levels of language abstraction proposed by the model (Maass, et al., 1989, 1995; Wigboldus, et al., 2000; Douglas \& Sutton, 2003). In the study and pretests, the descriptions were presented in random order. Below are the four levels of descriptions ordered from less to more abstract in terms of the linguistic category model (DAV, IAV, SV, and Adj).

\section{A Parker [BIC] pen sometimes does not write \\ A Parker $[B I C]$ pen sometimes does not work \\ I rate a Parker $[B I C]$ pen as not good \\ A Parker [BIC] pen has a mediocre quality}

Note that this study in itself provides an interesting extension of previous work on linguistic biases, by focusing on descriptions of participants own experiences with products, rather than descriptions of other people's behaviors (Maass, et al., 1989, 1995; Douglas \& Sutton, 2003, 2006; Webster, Kruglanski, \& Dwight, 1997). Pretest 1 studied the perceived quality and familiarity with the brands Parker and BIC, pretest 2 examined the perceived language abstraction, and pretest 3 the perceived valence.

\section{Pretests}

\section{Pretest 1}

A first pretest examined the perceived quality and familiarity with the brands Parker and BIC in a between-subjects design with fifty-seven students from the same pool of participants. Perceived quality was measured on a scale from (1) low quality to (9) high quality, and familiarity on a scale from (1) 'I am not at all familiar with this brand' to (9) 'I'm very familiar with this brand'. As anticipated, mean perceived quality ratings were higher for the Parker brand than for the BIC $(7.75$ vs. $6.83, t(55)=$ 
$3.41, p<.001)$, and Parker and BIC were rated as equally well-known brands (7.43 vs. 6.93, $t(55)=1.19, p=.239)$.

\section{Pretest 2}

A second pretest studied participants' perceptions of the language abstraction of the four descriptions with the ballpoint pen. Although these descriptions were formulated according to the linguistic category model, this does not necessarily imply that participants also perceived language abstraction to increase from the lowest level (DAV) to the second level (IAV), to the third level (SV), and to the fourth level (Adj).

Ninety-seven participants from the same subject pool as the study were shown the four descriptions of the ballpoint pen in random order (note that this mimics the presentation format in the actual experiment), and were asked to indicate the language abstraction for each description on a seven-point scale running from (1) concrete to (7) abstract. A repeated measure ANOVA with language abstraction of the description as a within subject variable with four levels (DAV vs. IAV vs. SV vs. Adj.) indicated that the manipulated level of language abstraction had a significant effect on the perceived language abstraction $(F(3,288)=41.21, p<.001)$. Follow-up planned comparisons confirmed that the DAV description (3.01) was perceived to be more concrete than the IAV description $(3.63, F(1,96)=16.74, p<.001)$, which in turn was seen as more concrete than the SV description $(5.00, F(1,96)=23.57, p<.001)$, which itself was perceived as marginally more concrete than the Adj description (5.56, $F(1$, $96)=3.78, p=.055)$. This means that a set of descriptions was created effectively, in which the intended level of language abstraction corresponds to the observed level of language abstraction.

\section{Pretest 3}

A third pretest examined the valence of the four descriptions. To rule out an alternative explanation whereby linguistic biases could be attributed to differences in the valence of descriptions, a set of descriptions were formulated in which the abstract descriptions in the set would not be more negative than the concrete descriptions. Twenty-three participants were shown the four descriptions of the ballpoint pen as in pretest 2, and were asked to rate them from (1) not at all negative to (4) very negative. A repeated measures ANOVA with language abstraction (DAV vs. IAV vs. SV vs. Adj) as a four-level within subject factor showed a marginally significant effect $(F(3,66)=$ 2.66, $p=.055)$. Average ratings were most negative for the most concrete statement (DAV; 2.43), and least negative for the most abstract description (Adj; 1.96). Negativity 
ratings for the intermediate levels IAV (2.13) and SV (2.17) were in-between. The difference between the most concrete and most abstract statement is significant $(F(1,22)$ $=6.53, p<.05)$, so within this set of descriptions there was a marginally significant inverse relationship between negativity and abstraction.

The second and third pretest thus suggest that I was successful in creating a set of four descriptions of this negative experience in which the observed abstractness corresponds to the intended language abstraction, and the negativity of the descriptions did not increase with increased language abstraction.

\section{Method}

\section{Design and Procedure}

Forty-three students were randomly assigned to a design in which brand attitude (unfavorable vs. favorable) was manipulated between subjects. Half of the participants were informed that the pen was made by Parker (a well-known high quality brand), while the other half was informed that the tested pen was a BIC pen (a well-known low-quality brand). The pen was presented in an unmarked silver casing. I invisibly damaged the points of the pens to make them write poorly. Participants were asked to write with the pen in order to test it. To make sure that participants indeed evaluated the pen, they were asked to indicate the writing color of the pen and the color of the casing from list of several alternatives. All of the participants provided the correct answers to these questions.

\section{Dependent Variables}

To obtain the main dependent variable, participants were asked how they would describe their experience to another student. Each participant was shown the pretested set of four product descriptions, and was then asked to choose the description that they would use if they were to communicate the product experience to someone else. This is the main dependent variable used in most research on language abstraction (Douglas \& Sutton, 2003; Maass, et al., 1989, 1995). In the analysis the four levels (which increase in terms of language abstraction) were coded from 1 to 4 : DAV $=1$, $\mathrm{IAV}=2, \mathrm{SV}=3$ and $\mathrm{Adj}=4$. Following earlier studies (Douglas \& Sutton, 2003; Maass, et al., 1989), these scores were treated as an interval scale. The averages were calculated within each of the two conditions. 


\section{Manipulation Checks}

As a manipulation check for product attitude, at the end of the test the participants were asked to identify the brand of the ballpoint pen from several alternatives. The manipulation check for product attitude revealed that all but two of the forty-three participants correctly remembered the brand of the pen. When these two participants are excluded from the analyses the effect remains significant. I also checked whether the product experience was viewed as negative, by asking participants to indicate whether the pen wrote well or not. Forty-one participants indicated that they thought the pen did not write well. When the data from the two participants who indicated the ballpoint pen wrote well were excluded, the effect remained significant.

After an open-ended suspicion probe, participants were debriefed and thanked. None of the participants in any of the studies of this dissertation indicated awareness of the processes underlying their answers in the dependent variables, the systematic variation in their language use, or the purposes of the studies.

\section{Results}

An ANOVA on the use of language abstraction with brand attitude (favorable vs. unfavorable) as independent variable revealed the expected difference in language use $(\mathrm{F}(1,41)=8.13, p<.01)$. In line with Hypothesis 1a, the negative experience was described in more abstract terms by participants with an unfavorable brand attitude (BIC; 3.25) than by participants with a favorable brand attitude (Parker; 2.32).

\section{Discussion}

This study reveals that consumers who have a negative experience with a product describe this experience in more abstract terms when they hold a less favorable a priori attitude toward the brand. This study provides a first demonstration of linguistic expectancy bias in the context of word of mouth. Here a set of descriptions was used that allowed us to rule out an alterative explanation, in which participants simply choose more negative descriptions to describe a less favorable brand. More specifically, a set of descriptions was created in which the negativity of the descriptions did not increase with the level of abstraction of the descriptions.

Study 2.2 seeks to generalize this effect to another form of word of mouth, in which consumers describe the experience of another consumer. To further address the possible confound between valence and abstraction, Study 2.2 uses a design in which the valence of descriptions is manipulated so that it either decreases or increases with 
the level of language abstraction. By examining the interaction between valence and abstraction, I can assess the extent to which these factors influence language use.

\title{
Study 2.2: Language Abstraction and Valence
}

This study examined whether or not systematic language use is driven by a process in which consumers seek to describe product experiences in such a way that the valence of the description matches their a priori attitude toward the product. To this end, not one but two sets of descriptions of one product experience were carefully constructed, in which the direction of the correlation between language abstraction and valence was manipulated.

This study focused on a negative product experience, and it showed participants a short video clip in which a person cut himself while shaving. The favorability of participants' attitudes toward the brand was manipulated by a brief description of the brand of razors. Participants were then asked how they would describe this experience to another consumer. To this end participants were provided with one of two sets of descriptions. In the first set of descriptions, valence and language abstraction were positively correlated. This means that an increase in language abstraction was paired with an increase in negativity of the statements. This corresponds to the "natural" or "common" situation, where abstract descriptions of a negative event are generally more negative than concrete descriptions of the same event. In the second set of descriptions, valence and language abstraction were negatively correlated, so that an increase in language abstraction was paired with a decrease in negativity. The two sets of product descriptions according to the four levels of the linguistic category model are presented below, and described in increasing order (from concrete to abstract: DAV, IAV, SV, and Adj). Both sets consist of descriptions that could be encountered in real life, and use common language.

\author{
Set 1: Positive correlation between abstraction and negativity \\ Jack shaves with Shaft razor blades \\ Jack damages his skin with Shaft razor blades \\ Jack finds shaving with Shaft razor blades painful \\ Shaft has bad razor blades
}




\section{Set 2: Negative correlation between abstraction and negativity \\ Jack cuts his skin open with Shaft razor blades \\ Jack hurts himself with Shaft razor blades \\ Jack finds shaving with Shaft razor blades unpleasant \\ Shaft has mediocre razor blades}

Note that this study provides a crucial test of the mechanism that underlies the linguistic bias that this chapter is studying. Hypothesis 1a proposes that consumers with an unfavorable brand attitude use more abstract language to describe this negative experience than consumers with a favorable brand attitude. If systematic language use in word of mouth were in part driven by a correlation between description valence and abstractness, I should find a significant interaction between product attitude and description set: the biases in language abstraction would be enhanced in the set where abstraction was paired with an increasing negativity of the descriptions (set 1), and reversed, or at least greatly attenuated, in the set where abstraction was paired with decreasing negativity (set 2). If, on the other hand, a main effect of attitude (unfavorable over favorable) and a non-significant attitude $x$ description set interaction emerges, this would imply that participants with an unfavorable (vs. favorable) brand attitude choose more abstract descriptions, regardless of the negativity of the statements.

\section{Pretests}

\section{Pretest 1}

A first pretest examined the perceived language abstraction of the two sets of descriptions. This was done in the same manner as pretest 2 for Study 2.1. Forty-four participants were shown one of the two sets of four descriptions, and were asked to rate the language abstraction of each description in the set, using a scale from (1) concrete to (7) abstract. A repeated measures ANOVA for the set of descriptions with a positive correlation between valence and language abstraction confirmed that the manipulated language abstraction of the description had a significant effect on the perceived abstractness $(F(3,129)=60.02, p<.001)$. Follow-up planned comparisons showed that the DAV description (1.89) was rated as more concrete than the IAV description $(2.57, F(1,43)=4.85, p<.05)$, which in turn was rated as more concrete than the SV description $(4.73, F(1,43)=51.92, p<.001)$, which itself was rated as more concrete than the Adj description $(6.02, F(1,43)=13.50, p<.01)$. For the set of 
descriptions with a negative correlation between valence and language abstraction, a similar analysis also revealed an effect of manipulated language abstraction on perceived language abstraction $(F(3,129)=65.24, p<.001)$. Planned comparisons $(F(1,43)>10, p<.01)$ showed that the four levels differed significantly in perceived abstraction, and increased from DAV (1.57) to IAV (3.39), to SV (4.70), to Adj (6.05). In conclusion, these results show that two sets of descriptions increasing in language abstraction were successful created.

\section{Pretest 2}

In a second pretest the valence of the descriptions was assessed by asking 117 participants to rate the descriptions on a scale from negative (1) to positive (5). For the analyses, these ratings were reversed so that higher scores correspond to more negative statements. An ANOVA with the language abstraction of the statement (DAV vs. IAV vs. SV vs. Adj) and type of description (increasing vs. decreasing negativity) as between subject variables on the valence of the statements revealed an interaction effect $(F(3,109)=12.39, p<.001)$, and no main effects $(F<1.5)$. In the set of descriptions with a positive correlation between abstraction and negativity (set 1) which was constructed to have a negativity increase with abstractness, more abstract statements were indeed rated as more negative, $F(1,52)=33.82, p<.001$ (the mean negativity scores from concrete to abstract were 3.21, 3.94, 4.45, and 4.29 respectively). Moreover, the set of descriptions with a negative correlation between abstraction and negativity displayed a decrease in the negativity of the statements with an increase in the level of abstraction of the descriptions, $F(1,57)=6.26, p<.05$ (the mean negativity scores from concrete to abstract were 4.40, 4.21, 3.76, and 3.87, respectively). Thus, my manipulation was successful: in the first set of descriptions, the negativity of the description increases with the level of abstraction as provided by the linguistic category model, whereas negativity decreases with abstraction in the second set of descriptions.

\section{Method}

\section{Design and Procedure}

One hundred and fifty-five students were randomly assigned to a 2 (product attitude: unfavorable vs. favorable) x 2 (set of descriptions: increasing vs. decreasing negativity) 
between subjects design. The data of two participants was not included in the analyses as they did not follow the instructions provided by the experiment leader.

Product attitude was manipulated by means of the following short product description (manipulation for negative attitude is presented within the brackets):

'Imagine that you are very positive [negative] about Shaft razor blade. You have used Shaft razor blades yourself and was [dis]satisfied with them. Shaft makes razor blades for both men and women.'

Product experience was presented by means of film clips of about ten seconds showing a student who cuts himself while shaving. This film clip provides a good portrayal of a consumer experience, which is more lively and realistic than the vignettes or cartoons that are used in previous research on language abstraction.

\section{Dependent Variables}

As in Study 2.1, the participants were shown a set of four descriptions of the product experiences. Depending on the condition they were in, they were shown either the set with the positive or the set with the negative correlation between language abstraction and negativity. This study contained two dependent variables. The first dependent variable is identical to the one used in the first study: participants were asked to choose the description that they would use if they were to communicate the product experience to someone else ('Use of Language Abstraction'). The four levels were coded using a scale from 1 to 4 . For the second dependent variable, labeled 'Abstraction Index', participants were asked to rate the extent to which each of the four descriptions was appropriate for sharing the product experience with someone else. This variable provides a more fine-grained indicator of participants' preference for more concrete or more abstract descriptions. For the second dependent variable, each description was rated on a seven-point scale, ranging from not at all (1) to very appropriate (7). An abstraction index was calculated based on the descriptor level coding (as applied in the first dependent variable) and participants' appropriateness ratings $\left(r_{D A V} ; r_{I A V} ; r_{S V} ; r_{A d j}\right.$ respectively $)$, yielding a normalized score between 1 and 4 (Wigboldus, Spears, \& Semin, 2005):

$$
\text { Abstraction Index }=\frac{\left(1 \times r_{D A V}\right)+\left(2 \times r_{I A V}\right)+\left(3 \times r_{S V}\right)+\left(4 \times r_{A d j}\right)}{\left(r_{D A V}+r_{I A V}+r_{S V}+r_{A d j}\right)}
$$


At the end of the study the participants also rated to valence of the descriptions on a five point scale from negative (1) to positive (5). In this section of the study, the descriptions were presented separate to the participants, and intermixed with filler statements. After an open-ended suspicion probe, participants were debriefed and thanked.

\section{Results}

\section{Use of Language Abstraction}

An ANOVA with product attitude (unfavorable vs. favorable) and set of descriptions (increasing vs. decreasing negativity) produced a main effect of attitude $(F(1,148)=$ 9.03, $p<.01)$, that is in line with Hypothesis 1a: participants with a unfavorable product attitude (2.21) described the negative experience more abstractly than participants with a favorable attitude (1.77), see Figure 1 for the means and standard errors. The analyses also showed a main effect of set of descriptions, which suggests that participants chose more abstract descriptions in the set of descriptions in which there was a positive (vs. negative) correlation between abstraction and negativity (2.35 vs. $1.65, F(1,148)=24.17, p<.001)$. Note that this result is not germane to the hypothesis, as it has no bearing on the relationship between language abstraction and the fact that experiences are congruent or incongruent to a priori brand attitudes. More important is the fact that there was no significant attitude $x$ set of descriptions interaction effect $(F<1)$. Furthermore, when the valence ratings of the descriptions was entered as a covariate, its effect on language abstraction was not significant $(F<$ 1 ), and the previously mentioned effects remained unaltered. In other words, the valence ratings did not mediate the effect of attitudes on language abstraction. 


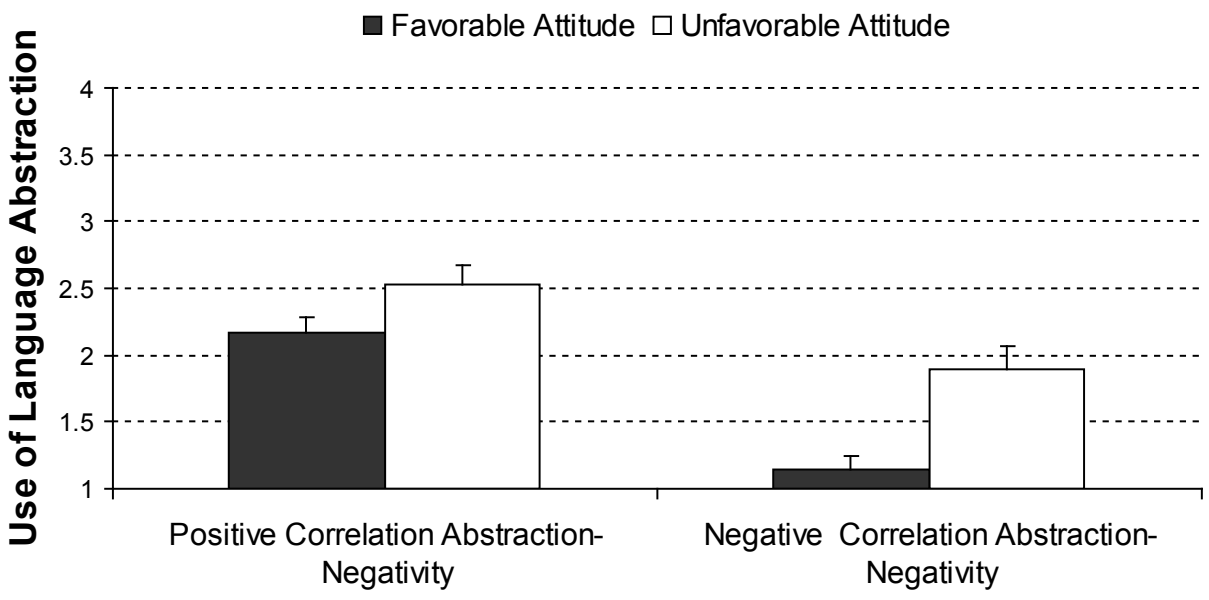

Figure 1.

Study 2.2. Effect of product attitude and set of descriptions (positively and negatively correlated descriptions between abstraction and negativity) on the Use of Language Abstraction: Mean (SE)

\section{Abstraction Index}

Similar findings were obtained with the abstraction index, which was the second dependent variable in this study. In line with the predictions, the ANOVA revealed a main effect of attitude $(F(1,148)=15.70, p<.001)$. An unfavorable product attitude (2.37) made participants rate more abstract descriptions as more appropriate compared to a favorable attitude (2.04), see Figure 2. There was also a main effect of set of descriptions $(F(1,148)=47.00, p<.001)$, but the attitude $\mathrm{x}$ set of descriptions interaction was not significant $(F<1)$. As with the first DV, including the valence ratings for the descriptions $(F<1)$ as a covariate did not affect the results. 
$\square$ Favorable Attitude $\square$ Unfavorable Attitude

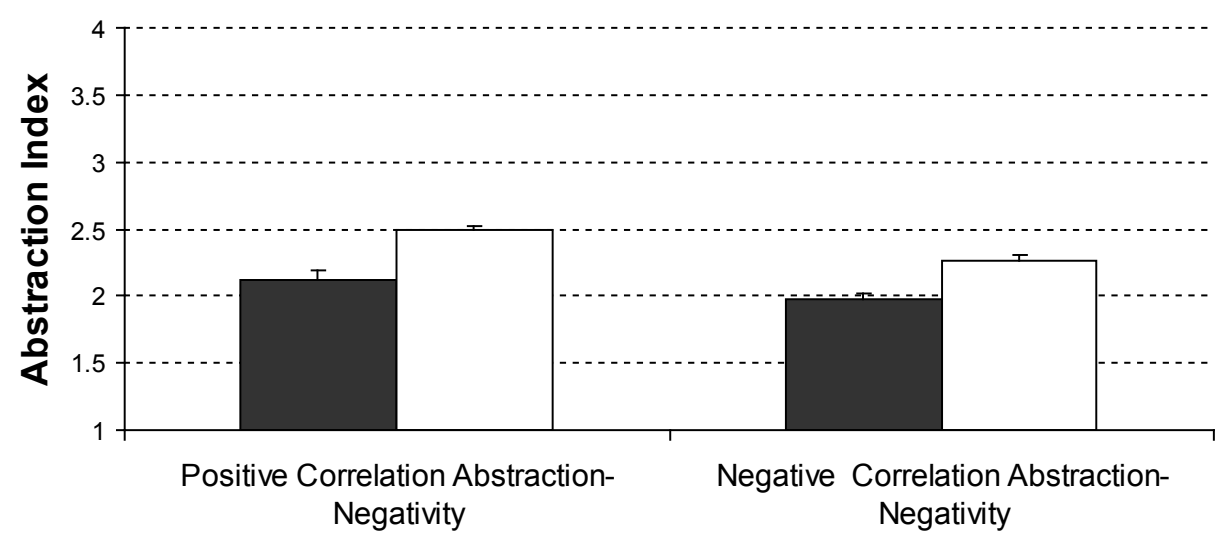

Figure 2.

Study 2.2. Effect of product attitude and set of descriptions (positively and negatively correlated descriptions between abstraction and negativity) on the Abstraction Index: Mean (SE)

\section{Discussion}

The results of this study converge with those of Study 2.1, and confirm Hypothesis 1a. Consumers described negative product experiences in a more abstract manner when they have an unfavorable rather than a favorable a priori brand attitude. Importantly, this study also shows that this systematic variation in language use is not driven by differences in the valence of abstract and concrete descriptions. Note that I do not argue that there is no positive association between language abstraction and valence. As explained in the theoretical framework, this correlation is inherent to the model. The present study, however, shows that this correlation is not responsible for the observed variation in language abstraction in descriptions of product experiences. Although some previous studies (Douglas \& Sutton, 2003, Study 5; Maass, et al., 1989, Study 1) have suggested that valence does not mediate the effects of language abstraction, the present study is the first to confirm this by experimentally manipulating the relationship between the valence and abstraction level of descriptions.

Studies 2.1 and 2.2 examined the use of language abstraction in negative word of mouth, which is seen as having a larger impact on consumers than positive information (Mittal, et al., 1998). The following four studies will look at negative as well as positive word of mouth. The next study will examine the effects of positive and 
negative product experiences on the language abstraction of descriptions for experiences with a variety of products, ranging from a fast moving consumer good to a technological device. In line with Hypothesis 1, negative product experience are expected to be communicated more abstractly by a consumer with an unfavorable (vs. a favorable) product attitude (H1a), while the positive product experiences are expected to be communicated more concretely by a consumer with an unfavorable (vs. a favorable) product attitude $(\mathrm{H} 1 \mathrm{~b})$. This should produce a significant interaction between product attitude and product experience.

\section{Study 2.3: Language Abstraction in Word of Mouth}

\section{Method}

\section{Design}

One hundred forty-four students were randomly assigned to a counterbalanced design with 2 (product attitude: unfavorable vs. favorable) x 2 (product experience: negative vs. positive) x 4 (product category: communication network, yogurt, T-shirt, and MP3 player). All factors were within subject variables. For each product the participants were exposed to a different combination between product attitude and product experience. Thus, the participants were exposed to all four combinations between the variables product attitude and experience, and to all four products. The factor product category was a repeated variable for replication purposes; there were eight different combinations between the four products and the product attitudes and experiences interactions. The products were shown in random order and were intermixed with two filler products.

\section{Procedure}

Product attitude was manipulated by means of a short product description as in Study 2.2. For Fresco yogurt (a fictitious brand), the manipulation of product attitude was for instance (manipulation for negative attitude is presented within the brackets):

'Imagine that you are very positive [negative] about Fresco yogurt. You tried Fresco yogurt and you really liked [disliked] it.' 
Product experiences were presented by means of film clips of about ten seconds showing a student who has a favorable or unfavorable product experience, as in Study 2.2. For instance, the Fresco yogurt clips showed a student who tastes Fresco yogurt. Then, she either smiles, approvingly nods and eats another spoonful (positive experience) or frowns, shows disgust, and pushes the bowl of Fresco yogurt away (negative experience)(see Figure 3 screenshots of experiences). The participants were shown a positive or a negative experience film clip per product.
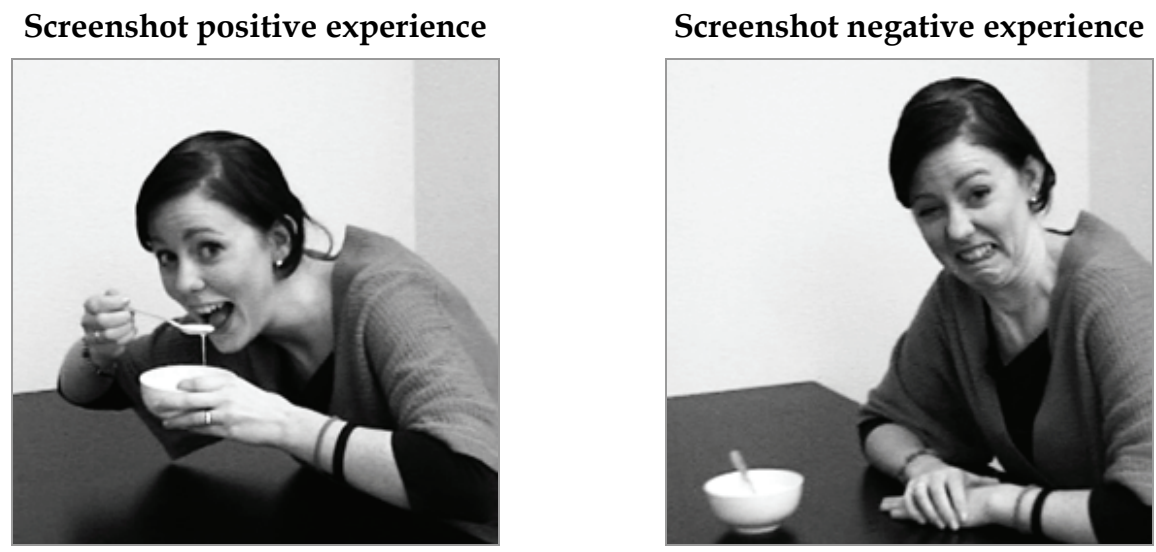

Figure 3.

Study 2.3. Screenshots of film clips of positive and negative experiences with product yoghurt.

\section{Dependent Variables}

After the product film clip, the participants were exposed to four descriptions of the positive [negative] product experience according to the four levels of the linguistic category model (Maass et al. 1989, 1995). The descriptions of the experiences are presented in the Appendix A. As in Study 2.2, the participants were asked to choose the description that they would use if they were to communicate the product experience to someone else ('Use of Language Abstraction'), and they rated all four descriptions on the extent to which they were an appropriate description of the product experience ('Abstraction Index'). After an open-ended suspicion probe, participants were debriefed and thanked. 


\section{Results}

\section{Use of Language Abstraction}

The data on the 'use of language abstraction' was analyzed with a repeated measures ANOVA with product attitude (unfavorable vs. favorable), and product experience (negative vs. positive) as within subjects variables, and product as covariate. The repeated measures analyses yielded solely a significant experience $\mathrm{x}$ attitude interaction-effect $(F(1,142)=15.80, p<.001)$ (main effects and interaction effects with product were not significant). Product experiences that were congruent with the product attitude were communicated more abstractly than incongruent experiences (3.11 vs. 1.91). Planned contrasts showed that, in line with Hypothesis 1a, negative experiences were described more concretely by participants with a favorable (vs. unfavorable) attitude towards the brand $(F(1,142)=80.10, p<.001)$. In line with Hypothesis $1 \mathrm{~b}$, positive product experiences were described more abstractly by participants with a favorable (vs. unfavorable) attitude towards the brand $(F(1,142)=$ $113.80, p<.001)$. Means and standard errors are presented in Figure 4.

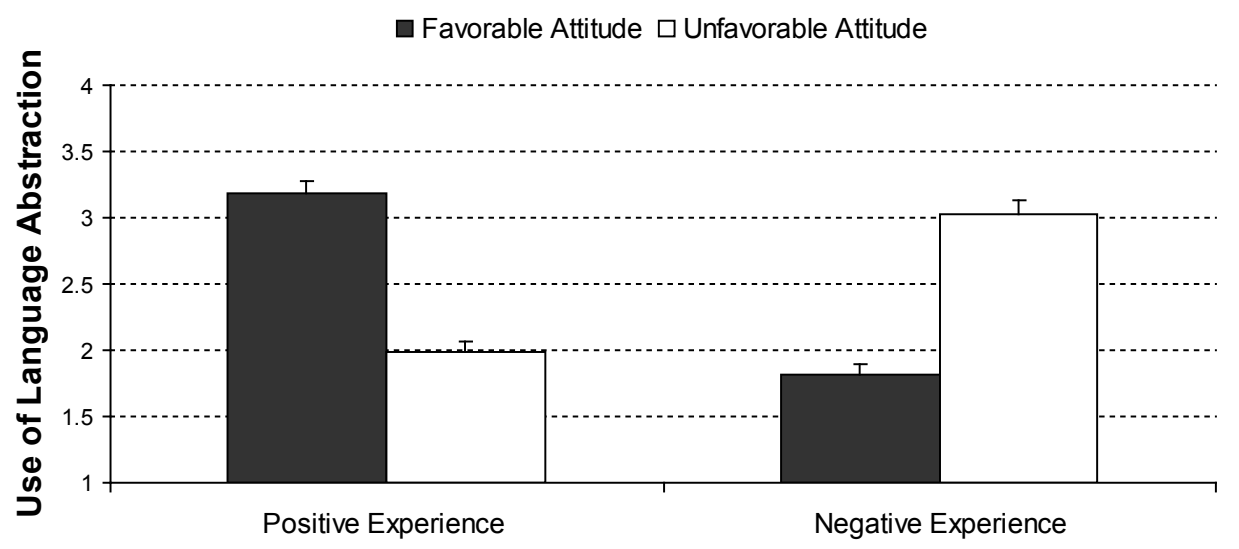

Figure 4 .

Study 2.3. Effect of product attitude and product experience on the Use of Language Abstraction: Mean (SE). 


\section{Abstraction Index}

Similar results were obtained with the abstraction index as the dependent variable in a repeated measures ANOVA with product experience and product attitude as between subject variables and product as covariate: a main effect of attitude $(F(1,142)=10.50, p$ $<.01)$, an attitude $x$ product interaction effect $(F(1,142)=8.81, p<.01)$, and an attitude $x$ experience effect $(F(1,142)=28.78, p<.001)$ (no other effects were significant). As expected, the abstraction index was higher for experiences that were congruent with one's attitude than for experiences incongruent with one's attitude (2.63 vs. 2.20). In line with Hypothesis 1a, the abstraction index for negative experiences was lower for participants with a favorable (vs. unfavorable) product attitude $(F(1,142)=102.64, p<$ .001). In line with Hypothesis 1b, the abstraction index for positive experiences was higher for participants with a favorable (vs. unfavorable) product attitude $(F(1,142)=$ 105.83, $p<.001$ ), see Figure 5. These results replicate the data pattern of the first dependent variable (Use of Language Abstraction).

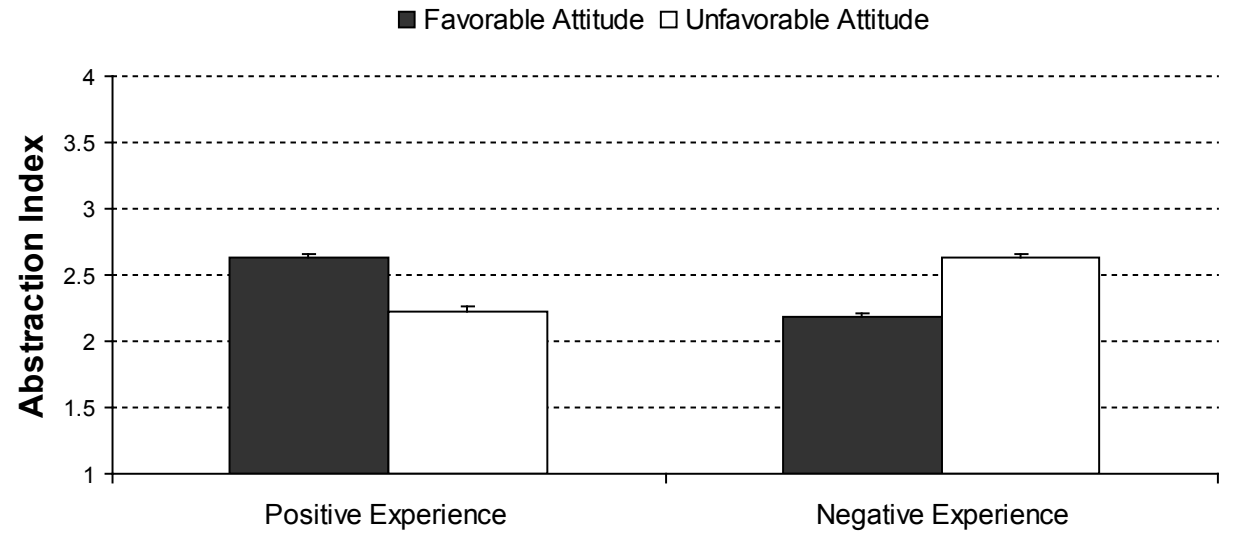

Figure 5.

Study 2.3. Effect of product attitude and product experience on the Abstraction Index: Mean (SE).

\section{Discussion}

This study provided support for both Hypotheses $1 \mathrm{a}$ and $1 \mathrm{~b}$. The results indicate that people use more abstract language when they engage in word of mouth about product experiences that are congruent to their a priori attitudes. This extends the first two studies by showing that this finding also pertains to positive product experiences. More specifically, negative product referrals were communicated more concretely by 
participants with a favorable compared to an unfavorable product attitude, and positive word of mouth was communicated more abstractly by participants with a favorable product attitude compared to an unfavorable attitude. Note that this finding of a cross-over interaction rules out that participants always choose to describe experiences with unfavorable brands in a more abstract manner.

In Study 2.3, product attitude was manipulated as part of the experimental procedures. Study 2.4 will enhance the realism of the research by using existing brands that were pre-selected to represent brands towards which participants had an unfavorable or favorable attitude.

\section{Study 2.4: Language Abstraction and Existing Brand Attitudes}

In this study participants were asked to imagine that the film clip they were going to see depicted a product experience that happened with a product of the assigned brand attitude. For favorable product attitude, the brands Apple iPod (MP3 player) and Nike (clothing) were used. For unfavorable attitudes, the brands Funstick (MP3 player) and Wibra (clothing) were used.

\section{Pretest}

In a pretest $(\mathrm{N}=17)$, the brands Apple, Nike, Funstick and Wibra (intermixed with several filler products) were rated on a semantic differential scale from (1) negative to (9) positive. As anticipated, Apple iPod was rated more positively than Funstick (7.06 vs. 3.56), $(t(16)=27.00, p<.001)$, and Nike was rated more positively than Wibra $(6.63$ vs. 3.31), $(t(16)=28.26, p<.001)$.

\section{Method}

One hundred fifty-three students were randomly assigned to a 2 (brand attitude: unfavorable vs. favorable) $\times 2$ (product experience: positive vs. negative) $\times 2$ (product category: MP3 player vs. T-shirt) within subjects design. In this study, a counterbalanced design was used for attitude and experience, where each participant experienced two of the four combinations between attitude and experience, one combination for each product. Products were presented at random order. The data for each product were analyzed in separate ANOVAs. The experimental procedures and major dependent measures were identical to those in Study 2.3. As in Study 2.3, 
participants then chose one of the four descriptions of the product experience ('Use of Language Abstraction') and rated all four descriptions on appropriateness ('Abstraction Index').

\section{Results}

Use of Language Abstraction

Two ANOVAs with product attitude (unfavorable vs. favorable) and product experience (negative vs. positive) regarding the use of language abstraction yielded a main effect of product experience for the T-shirt data $\left(M_{\text {positive }}=2.42\right.$ vs. $M_{\text {negative }}=$ $2.03),(F(1,149)=8.37, p<.01)$. No other main effects were found. More importantly, the analyses showed attitude $x$ experience interaction effects for both products: MP3 player $(F(1,149)=7.77, p<.01)$ and T-shirt $(F(1,149)=9.79, p<.01)$. As expected, the product experiences were communicated more abstractly if they were congruent with a priori brand attitudes - for MP3 player (3.01 vs. 2.75$),(t(151)=7.69, p<.01)$, and for T-shirt $(2.44$ vs. 2.01$),(t(151)=9.29, p<.001)$.

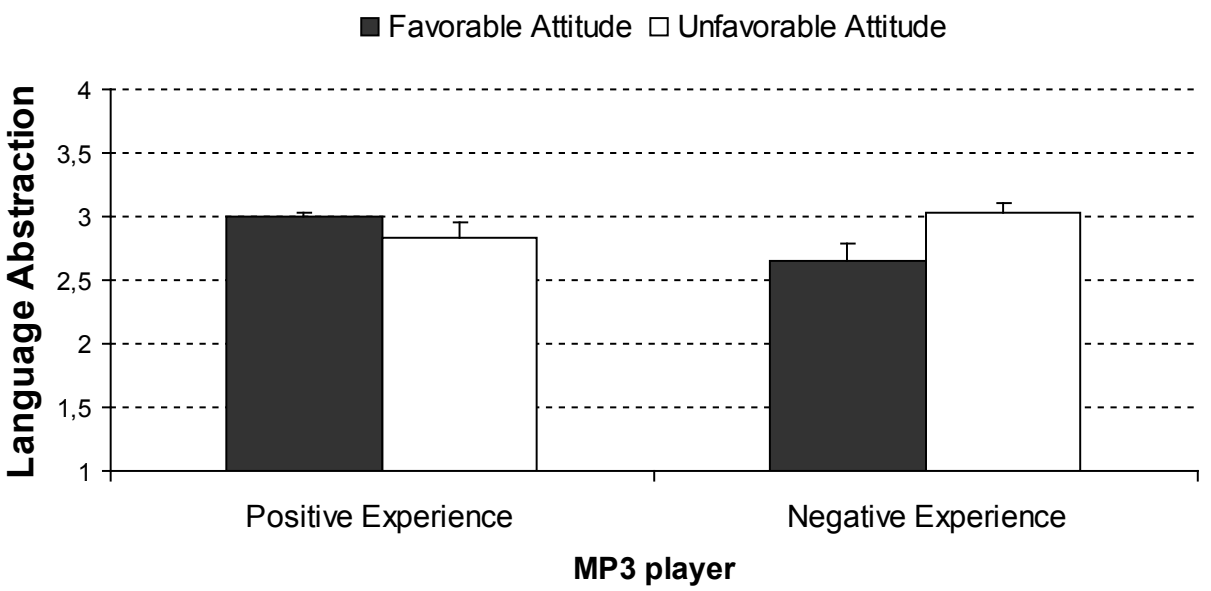

\section{Figure 6a.}

Study 2.4. Effect of existing attitude and experience with MP3 player on the Use of Language Abstraction: Mean (SE).

Hypothesis 1a was confirmed for both products: negative product experiences were communicated more concretely by participants with a favorable brand attitude than by participants with an unfavorable attitude - for MP3 player $(F(1,149)=7.66, p<.01)$, 
and for T-shirt $(F(1,149)=5.86, p<.05)$; see Figure $6 \mathrm{a}$ and $6 \mathrm{~b}$ for means and standard errors. In line with Hypothesis $1 b$, planned contrasts showed that the positive experience with the T-shirt was described more abstractly for a favorable rated brand than for an unfavorable rated brand $(F(1,149)=4.03, p<.05)$. For the positive experience with the MP3 player, brand attitude did not significantly influence use of language abstraction $(F(1,149)=1.36, p=.245)$.

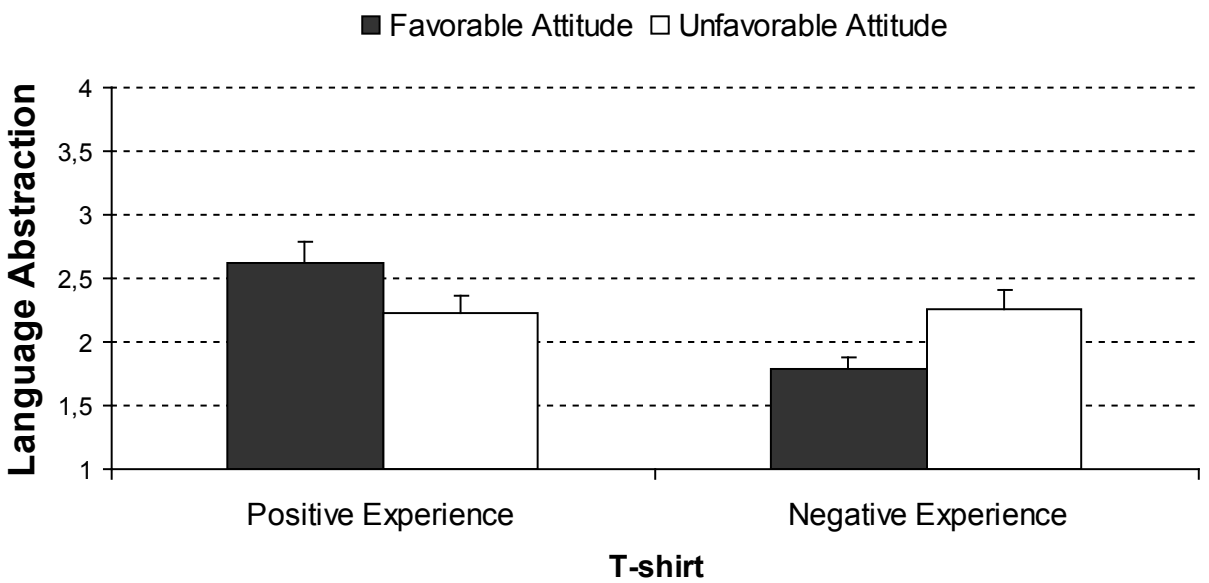

Figure 6b.

Study 2.4. Effect of existing attitude and experience with T-shirt on the Use of Language Abstraction: Mean (SE).

\section{Abstraction Index}

For the abstraction index, similar ANOVAs were conducted with product experience and product attitude as between-subjects factors. The expected attitude $x$ experience effects emerged for both products: MP3 player $(F(1,149)=6.47, p<.05)$ and T-shirt $(F(1,149)=5.71, p<.05)$. The analyses also revealed a main effect of attitude for MP3 player $\left(M_{\text {favorable }}=2.48\right.$ vs. $\left.M_{\text {unfavorable }}=2.62\right),(F(1,149)=5.17, p<.05)$ and a main effect of experience for T-shirt $\left(M_{\text {positive }}=2.50\right.$ vs. $\left.M_{\text {negative }}=2.23\right),(F(1,149)=27.98, p<.001)$; no other main effects were significant. As anticipated, the abstraction index was higher for product experiences that were consistent (vs. inconcsistent) with brand attitude: MP3 player (2.62 vs. 2.47), $(t(151)=6.24, p<.05)$, and T-shirt (2.42 vs. 2.30$),(t(151)=$ $4.72, p<.05)$. Planned contrasts indicated that in line with Hypothesis 1a, negative product experiences for both products received higher abstraction indices for unfavorably rated brands than for favorably rated brands: MP3 player $(F(1,149)=5.76$, 
$p<.05)$, and T-shirt $(F(1,149)=12.09, p=.001)$; see Figure $7 \mathrm{a}$ and $7 \mathrm{~b}$ for means and standard errors. Unfortunately, the positive product experiences did not significantly differ on the abstraction index.

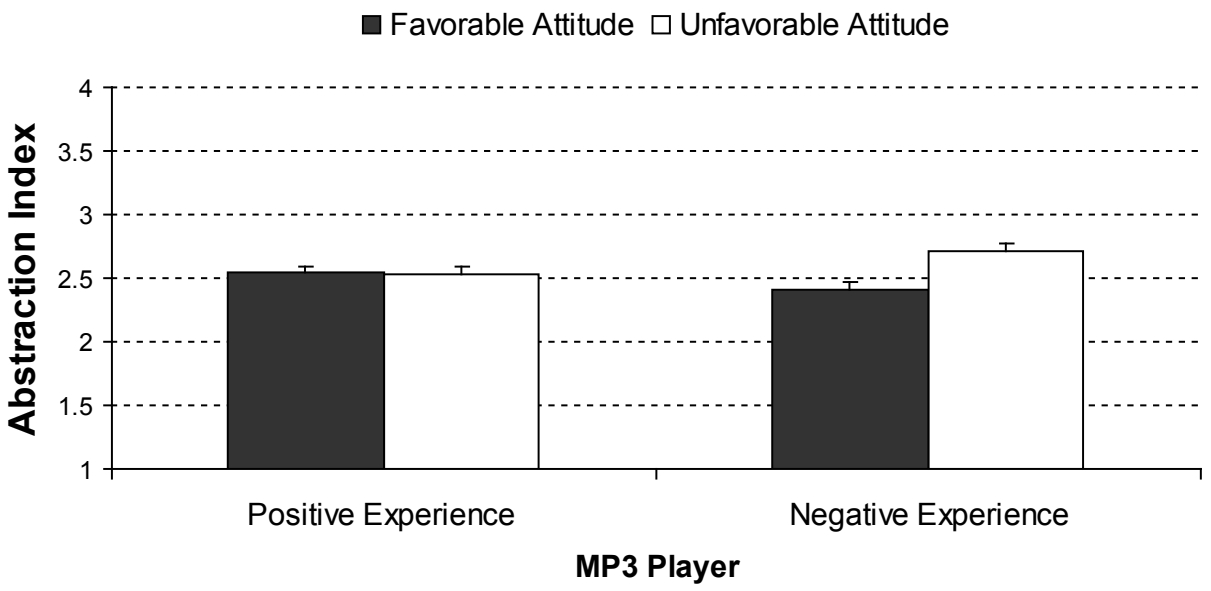

\section{Figure 7a.}

Study 2.4. Effect of existing attitude and experience with MP3 player on the Abstraction Index: Mean (SE).

口 Favorable Attitude $\square$ Unfavorable Attitude

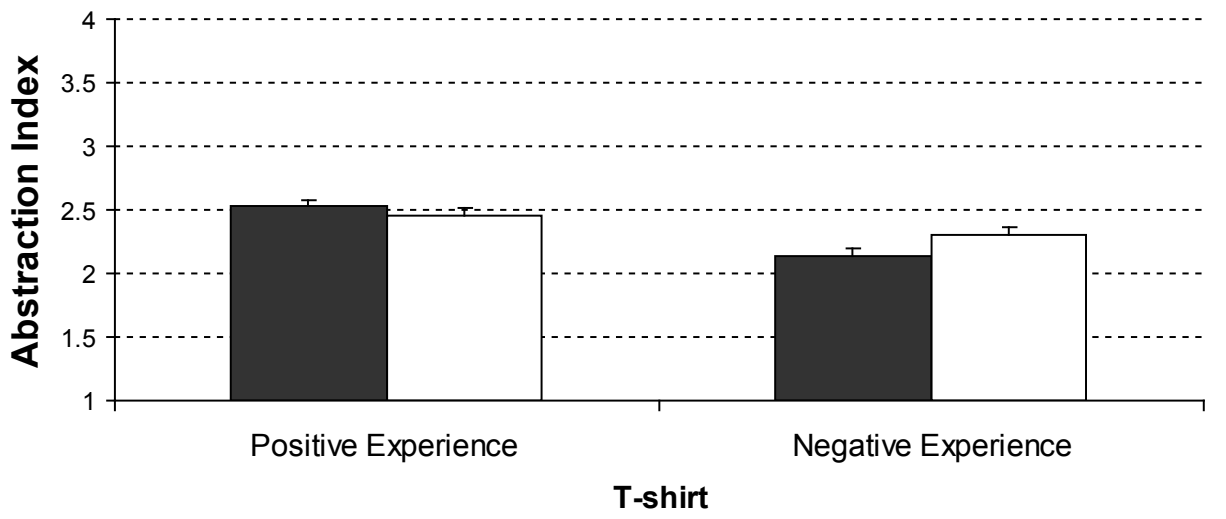

\section{Figure $7 \mathrm{~b}$.}

Study 2.4. Effect of existing attitude and experience with T-shirt on the Abstraction Index: Mean (SE). 


\section{Discussion}

Study 2.4 replicated the findings of Study 2.3 and demonstrated the systematic use of language abstraction in word of mouth about existing brands. Product experiences are communicated more abstractly when they are consistent with the brand attitude. This increases the realism of the studies of this dissertation and the practical relevance of the findings.

In the Studies 2.1 through 2.4 participants rated and selected predetermined descriptions as a response mode, a method that is commonly applied in research on language abstraction (Douglas \& Sutton, 2003; Maass, et al., 1989). Because this method restricts the language consumers can use to describe their experiences, Study 2.5 uses a different format, and asks participants to describe a product experience in their own words. Moreover, this study uses mediation analyses to test whether the interaction effect of product attitudes and experiences on the language abstraction of participants' product descriptions is mediated by the valence of participants' product descriptions.

\section{Study 2.5: Language Abstraction in Open Product Descriptions}

This study aimed to extend the applicability of this chapter's findings by examining language abstraction in a setting where participants described a product experience in their own words. To assess the abstraction level of the language that is used by participants, a coding scheme developed by Semin and Fiedler (1988) is used, which was successfully applied in prior studies on linguistic abstraction (Webster, et al., 1997; Wenneker, et al., 2005; Wigboldus, et al., 2000). As in Study 2.1 and 2.4, familiar existing brands were used to manipulate product attitudes. More specifically, product attitudes were manipulated by asking participants to imagine that a portrayed (negative or positive) experience involved one of their most (or least) favorite brands in the category.

\section{Method}

\section{Design}

Sixty-seven students were randomly assigned to a 2 (brand: unfavorable vs. favorable) x 2 (product experience: negative vs. positive) between-subjects design. The data of 12 participants was excluded from the analyses, because they did not provide adequate 
descriptions, and provided comments at a meta-level rather than describing the experience itself (e.g., when asked to write down how they would describe the experience, they said things like 'I would tell the truth' or 'I would not say anything about it').

\section{Procedure}

Depending on condition, participants were instructed to choose a clothing brand that they either did or did not like, and write this brand name on the questionnaire. Next, they were shown a "before-after" drawing (made by a professional artist), which showed a black T-shirt that either had remained perfectly black after washing it 40 times (positive experience) or had faded after five washes (negative experience), see Figure 8. Participants were asked to imagine having this experience with a shirt of their chosen brand, and to describe how they would communicate this product experience to another person. Finally, participants were probed for suspicion, debriefed, and thanked.

\section{Positive experience}

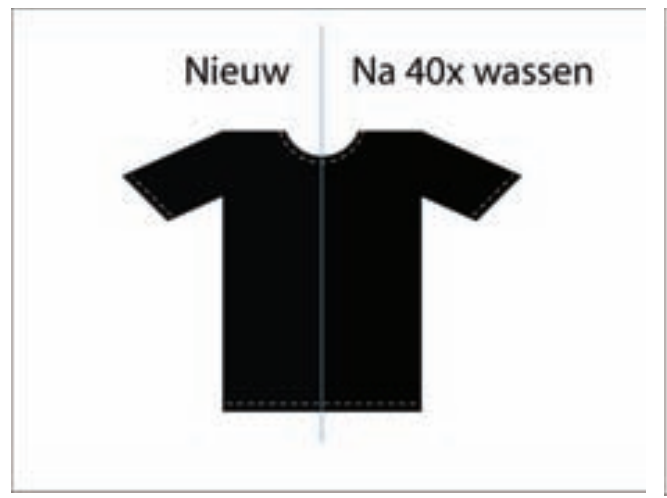

Negative experience

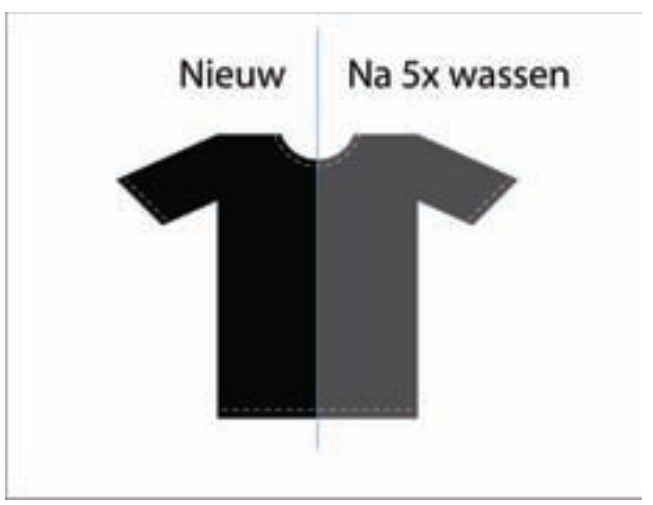

\section{Figure 8.}

Study 2.5 Positive and negative experience with a T-shirt.

\section{Dependent Variables}

Verbs and predicates in the open-ended descriptions were coded according to the linguistic category model (Semin and Fiedler 1988) by two independent judges who were blind to the experimental conditions. Inter-judge reliability was high $(r=.92)$. A linguistic abstraction index was computed for each participant by summing the number of occurrences of DAV's (coded as 1 as in Study 1), IAV's (multiplied by the 
IAV code of 2), SV's (multiplied by the SV code of 3), and Adj's (multiplied by the Adj code of 4) and dividing this sum by the total number of verbs and adjectives used by the participant (Semin \& Fiedler, 1989; Semin, et al., 2005):

$$
\text { Open Response Abstraction Index }=\frac{\left(1 \times f_{D A V}+2 \times f_{I A V}+3 \times f_{\mathrm{SV}}+4 \times f_{\mathrm{Adj}}\right)}{\left(f_{D A V}+f_{\mathrm{IAV}}+f_{\mathrm{SV}}+f_{\mathrm{Adj}}\right)}
$$

where $f_{\mathrm{DAV}}, f_{\mathrm{IAV}}, f_{\mathrm{SV}}$, and $f_{\mathrm{Adj}}$ represent the frequency of occurrences of each category. This normalized index ranges from 1 to 4 .

Afterwards, two other independent judges who were blind to the condition and hypotheses of the study rated the valence of the open-ended descriptions on a five point scale from negative (1) to positive (5). The inter-judge reliability was high $(r=$ .91).

\section{Results}

An ANOVA on the open response abstraction index with brand attitude (favorable vs. unfavorable) and product experience (positive vs. negative) as between subject factors revealed a main effect of experience in that positive experiences were described more abstractly than negative ones $(2.58$ vs. $2.16, F(1,51)=4.24, p<.05)$, and the expected interaction effect $(F(1,51)=6.95, p<.05)$, see Figure 9. Confirming Hypothesis 1, experiences that were congruent with brand attitude were communicated more abstractly than incongruent experiences (2.69 vs. 2.15$),(t(51)=2.46, p<.05)$. In line with $\mathrm{H} 1 \mathrm{a}$, the negative experience was described more concretely by participants with a favorable (vs. unfavorable) brand attitude (1.89 vs. $2.51, F(1,51)=2.77, p=.10)$. In line with Hypothesis $1 b$, planned contrasts revealed that the positive experience was described more abstractly by participants with a favorable (vs. unfavorable) brand attitude (2.91 vs. $2.39, F(1,51)=4.30, p<.05)$.

An ANOVA with product experience (positive vs. negative) and brand attitude (favorable vs. unfavorable) on the rated valence of the open-ended descriptions revealed main effects of product attitude (3.06 vs. $2.30, F(1,51)=6.16, p<$ $.05)$ and product experience $(4.06$ vs. $1.93, F(1,51)=6.16, p<.001)$, but no attitude $x$ experience effect $(F<1)$. In itself, this insignificant interaction effect suggests that valence does not mediate the interaction of attitude and experience on language abstraction. The correlation between language abstraction and valence was also non- 
significant (Pearson correlation .142). Indeed, a subsequent ANCOVA with product attitude and experience as factors, and language valence as a covariate revealed the expected attitude $x$ experience interaction effect on language abstraction $(F(1,50)=$ $6.66, p<.05)$. The absence of mediation was corroborated by an insignificant outcome of a Sobel test $(z=.27, p=.784)$.

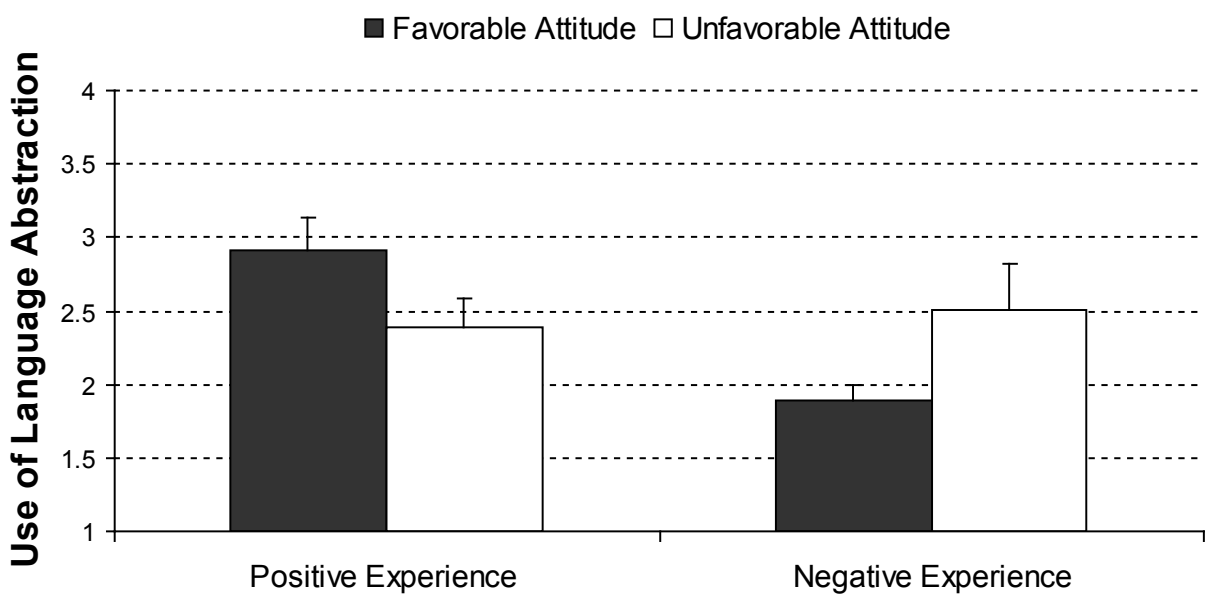

Figure 9.

Study 2.5. Effect of product attitude and product experience on the Use of Language Abstraction of open response word of mouth: Mean (SE).

\section{Discussion}

In this study, participants described product experiences in their own words, rather than picking a description from a set of alternatives. The fact that this study replicates previous results increases the practical relevance of the findings. For an example of language abstraction bias in this setting, one may compare descriptions of the positive experience between participants in the favorable and unfavorable brand conditions. A typical example of a description in the favorable T-shirt brand condition was: "the [brand] T-shirt is of top quality." In contrast, a typical example from the unfavorable brand condition was: "after 40 times washing, the [brand] T-shirt did not change".

Study 2.5 again tested for the possibility that the language used by the participants is caused by a tendency to use stronger valenced descriptions for experiences that are in line with their attitudes. As in Study 2, there was no evidence for the notion that language valence mediated the effect of product attitude on 
language abstraction. Using different methodologies, different products, and different dependent variables, Study 2.1, 2.2 and 2.5 provide strong evidence for the notion that consumers systematically vary language abstraction based on the extent to which product experiences are consistent with their a priori product attitudes, and show that this effect cannot be explained by differences in valence that may exist between concrete and abstract descriptions of an experience.

Up till now, consumers have been shown to systematically use more concrete or abstract product messages to communicate product experiences depending on if this experience was confirming or countering their product attitude. Moreover, this systematic use of language abstraction is found not to be solely driven by valence. What then does trigger consumers to be more concrete or abstract? The next study examines this underlying process and tests Hypothesis 2.

The goal of Study 2.6 is to reveal an important mediator in the effect of the (in)congruency between product attitudes and experiences on systematic variations in the use of language abstraction. As outlined before, I propose that these variations in language abstraction are caused by the fact that consumer's inferences about the cause of an experience are determined by their a priori expectations. If a product experiences is congruent with a consumers' a priori product attitude, then the consumer will more likely infer that the experience can be attributed to the product itself, and not to the user or the usage situation. This inference translates into the use of more abstract language to describe the experience, which communicates that an experience is more typical for a product or brand, and therefore is seen as more stable and enduring, and more likely to be repeated. In contrast, incongruent experiences are more likely to be attributed to the user or the usage situation, which favors the use of concrete language to describe the experience. Thus, it is hypothesized that the inferred cause of the experience mediates the effect of product attitude and experience on the systematic variations in language use.

\section{Study 2.6: Attribution Underlying the Systematic Use of Language Abstraction}

This study was designed to reveal an underlying process of systematic language use. In this study, product attitude and experience were manipulated and the use of language abstraction was measured as in previous studies. Additionally, the inferred 
cause of the product experience was measured. If this inferred cause of the product experience mediates the effect of product attitudes on language use, then by controlling for this inferred cause in the analyses the interaction effect of attitude and experience on the systematic variations in language abstraction should disappear.

\section{Method}

One hundred forty-four students were randomly assigned to a 2 (product attitude: favorable vs. unfavorable) x 2 (product experience: positive vs. negative) between subjects design. Product attitude and experiences were manipulated as in studies 2.3 and 2.4. To manipulate product experience, participants were shown a film clip in which a consumer had a positive or negative experience with a dishwasher. The descriptions of the positive and negative experiences according to the four levels of the linguistic model are presented in Appendix A. Participants were asked to choose the description that they would use if they were to communicate the product experience to someone else ('Use of Language Abstraction'). The inferred cause of the experience is measured by asking the participants to indicate whether they considered the product user or the brand to be the cause of the product experience from (1) the person caused the experience to (9) the brand caused the experience.

\section{Results}

\section{Use of Language Abstraction}

An ANOVA with 2 (product attitude: favorable vs. unfavorable) $\times 2$ (product experience: positive vs. negative) on the choice of description revealed main effects for experience $\left(M_{\text {positive }}=2.52\right.$ vs. $\left.M_{\text {negative }}=2.94, F(1,140)=8.21, p<.01\right)$, and an interaction effect $(F(1,140)=32.07, p<.001)$. Confirming Hypothesis 1a, negative experiences were communicated more concretely by participants with a favorable (vs. unfavorable) attitude towards the brand $(2.51$ vs. $3.36, F(1,140)=17.11, p<.001)$. As expected, the analyses revealed the opposite effects for the positive experience. In line with Hypothesis 1b, planned contrasts showed that positive experiences were communicated more abstractly by participants with a favorable (vs. unfavorable) attitude towards the brand (2.92 vs. $2.14, F(1,140)=14.99, p<.001)$. 


\section{Attribution Underlying Language Abstraction}

Following the procedure of Baron and Kenny (1968), the regression analyses with product experience and attitude and the interaction between experience and attitude on the 'use of language abstraction' revealed a main effect of experience $(b=-.206$, $t(140)=-2.86, p<.01)$, and an experience $\mathrm{x}$ attitude interaction effect $(b=.407, t(140)=$ $5.66, p<.001)$. The same regression on the 'inferred cause of the experience' demonstrated an experience $\mathrm{x}$ attitude effect $(b=1.42, t(140)=8.22, p<.001)$, and no main effects. When controlling for the effect of 'inferred cause of the experience' on the use of language abstraction, the regression analyses with product attitude, experience, inferred cause of the experience, and the experience-attitude interaction showed an effect of experience $(b=-.189, t(139)=-2.84, p<.01)$, an effect of inferred cause of the experience $(b=.164, t(139)=5.03, p<.001)$ and an experience $\mathrm{x}$ attitude effect $(b=.175$, $t(139)=2.17, p<.05)$. However, it is clear that this interaction effect after correction for the cause of the experience $(t=2.17)$ was much less strong than the interaction effect without correction $(t=8.22)$ (eta ${ }^{2}$ of the interaction effect went down from .186 to .033 - a reduction of $82 \%)$. A Sobel test indicated that the reduction in effect size attributable to the systematic variations in linguistic abstraction was statistically significant, $Z=4.26, p<.001$. These results provide support for Hypothesis 2.

\section{Discussion}

This study gained insight into the 'Why?' of systematic variations in linguistic behavior during word of mouth. If a product experience is congruent with a consumer's a priori product attitude, then the cause of the experience is attributed to the brand, and consumers use more abstract product descriptions. When the experience is incongruent with a consumer's a priori product attitude, then it is attributed to the usage situation, resulting in the use of more concrete language. The effect of the interaction between experience and attitude on variations in language abstraction is mediated by the attributed cause of the experience. More explicitly, up to $82 \%$ of the effect of product attitude on language use was explained by changes in the inferred cause of the event.

\subsection{General Discussion}

In spite of a large amount of research on word of mouth, there has been little attention on the language that consumers use to describe their product experiences to others. 
The first empirical chapter of this dissertation addressed this gap, and showed how the congruency or incongruency between product attitudes and experiences leads consumers to use more abstract or more concrete language when they describe their product experiences. To demonstrate the robustness of these findings a variety of manipulations for the independent variables were used, and a wide range of product categories were examined in the studies. I decided to focus on written word of mouth, since more and more experiences are shared via written messages, such as in text messaging, email, and blogs (Dellarocas, 2003; Hennig-Thurau, et al., 2004; Schau \& Gilly, 2003). This chapter provides three main findings that contribute not only to the extensive research on word of mouth but also to the literature on language abstraction.

First of all, this research reveals that language abstraction in word of mouth is systematically influenced by consumers' brand attitudes. Product experiences that are congruent with consumers' brand attitudes are communicated in more abstract terms than experiences that are incongruent with their brand attitude. While negative product experiences are communicated more abstractly by consumers with an unfavorable (vs. a favorable) product attitude, positive product experiences are communicated more concretely by consumers with an unfavorable (vs. a favorable) product attitude. This effect was generalized across a wide range of contexts. For example, in Study 2.1, participants described an actual experience with a product of a real brand, while in Studies 2.2, 2.3 and 2.4 they saw film clips showing another person having an experience with a product of a fictitious brand. Study 2.5 used a cartoon to help participants imagine a positive or negative experience with a familiar brand. Furthermore, this systematic language use operates not only when consumers rated or chose from pre-defined statements (Studies 2.1-2.4), but also when they described product experiences in their own words (Study 2.5).

Research on linguistic biases has so far described how language abstraction is influenced by social categories and stereotypes (e.g., gender, nationality). This dissertation is the first to apply the linguistic category model outside the context of (inter)personal behavior, and demonstrates that it affects the language that consumers use in word of mouth to describe their positive and negative product experiences. While this research focused on the congruence and incongruence between product attitudes and experiences, there may be other variables that influence the level of language abstraction in consumers' word of mouth. Examples worthy of mentioning may be product price and the store where the product is sold. For instance, a negative experience with a cheap product or one bought at a budget store confirm one's low 
expectations of this product and is probably described abstractly. Conversely, an outstanding performance of such a cheap product may feel as a lucky shot and be described concretely. The practical implications of these findings will be discussed in paragraph 5.4.1.

A second important contribution to the language abstraction literature is the consistent demonstration that the effect of product attitudes and experiences on language abstraction is not mediated by differences in the valence of the descriptions. Although the role of valence has been addressed in some previous work on language abstraction (Douglas \& Sutton, 2003, 2006), the evidence has not been conclusive. Using various approaches I control for possible effects of valence in several of the studies. Most importantly, Study 2.2 of this chapter is the first study in the literature to experimentally manipulate the correlation between the abstraction and valence of descriptions. This approach allowed me to conclusively demonstrate that the effects of brand attitudes and experiences on language abstraction are not driven by differences in valence between concrete and abstract language. This finding was confirmed by the results of the other studies of this chapter, where the valence of the descriptions was carefully held constant (Study 2.1), or was statistically controlled for (Study 2.5).

The third contribution is that the effect of product attitudes on the systematic use of language abstraction is mediated by the inferred cause of the experience. In previous research, there has been limited attention to the question of whether linguistic biases are indeed related to the extent to which descriptions focus on the actor (or in this case: the product), or on other, more situational facets of the event. Study 2.6 showed how the systematic variation of language abstractness in word of mouth is driven by the fact that experiences which are in line with one's product attitude are more likely to be attributed to the product than to the specific user or usage situation. Although this mediation is in line with Semin and Fiedlers (1988) theorizing, it had not been tested empirically before. This inference process mediates the interaction effect of product attitudes and experiences on language abstraction, and I argue that this mechanism is the main driver of the systematic use of language abstraction. 


\section{Chapter 3. Language Variation in Sender-Receiver Dyad}

\subsection{Strategic Use of Language Abstraction}

In word of mouth, consumers communicate brand, service, company and product related messages to other consumers. A variety of motivations may influence what consumers say to one another (Dichter, 1966; Sundaram, Mitra, \& Webster, 1998; Thurau, Gwinner, Walsh, \& Gremler, 2004). Main elements are the receiver to whom one communicates and the goal with which one communicates (Grice, 1975; Higgins, 1981; Schwarz, 1994). A common communication goal in word of mouth is to persuade other consumers of the superiority or inferiority of a product on one or more dimensions. For instance, consumers may try to improve their status by bragging about the performance of their newly-bought car (Feick \& Price, 1987). But also the goals to create a positive atmosphere, or help a neighbor by warning them about a disappointing movie are common. Related to the characteristics of the receiver of word of mouth, part of the strength of word of mouth lies in the possibility to adjust each and every product message to the needs of a unique receiver (Herr, Kardes, \& Kim, 1991). Next to general information such as age, gender and social-economical background, the opinion of a receiver about the product in question is of main importance. In this dissertation it is argued that a receiver's product attitude and the communication goal of the sender will influence the language of word-of-mouth messages.

Research in social psychology suggests that communication goals can affect the use of language abstraction. Several studies have shown that people are not aware of the abstraction level at which they describe an event, and their motivations for choosing a certain level do not seem to be cognitively accessible (Franco \& Maass, 1996, 1999; Maass et al., 1995; Von Hippel, Sekaquaptewa, \& Vargas, 1997; Webster, Kruglanski, \& Pattison, 1997). The systematic variations in language abstraction escape conscious access and are difficult to control. Notwithstanding that people have been shown to adapt the abstractness of their language in response to specific task instructions (Douglas \& Sutton, 2003; Wenneker, et al., 2005). For instance, Wenneker 
and colleagues (2005) instructed participants to describe an actor's behavior in a positive or negative way, and people respond to this instruction by providing more abstract descriptions of positive events, and more concrete descriptions of negative events. Although these findings may seem to contradict the notion that language abstraction occurs outside of the awareness of speakers, Wenneker pointed out that their findings do not necessarily imply that people are aware of the fact that they were adjusting the level of linguistic abstraction of their language in order to create a positive or negative impression. As such, their "...effects can be seen as intentional in the sense that they are strategic but as unintentional in the sense that people are (mostly) unaware of the tool they use..." (Wenneker, et al., 2005, p. 514).

These findings suggest that the language that consumers use to communicate positive and negative product experiences may not only be driven by their a priori expectations about these experiences, but also by essential elements in a senderreceiver dyad. This chapter examines the adaptive use of language abstraction in word of mouth to the two most central elements in word-of-mouth communication: the goal and target of the communication. More specifically, I focus on the adaption of language abstraction to the product attitude of a receiver, but also on the use of language abstraction to achieve a desired product attitude in the receiver through persuasive communication.

\subsection{Effect of Product Attitude of Receiver on Language Abstraction}

In the real world, people often are more or less aware of the attitudes and opinions of the ones they talk to, and (spontaneously) adapt their messages to the beliefs and opinions of the receiver (e.g., Fussell \& Krauss, 1989). In fact, successful communication even requires that communicators tailor their speech to the recipient's characteristics and knowledge (Grice, 1975; Higgins, 1981; Schwarz, 1994). Higgins and Rholes (1978), for example, found that people adapt the evaluative tone of their descriptions to the recipient's attitude towards the described person or object. In parallel, Higgins (1981) suggested that information that is incongruent with a receiver's viewpoints on an issue is generally presented in a manner that reduces the possibility of dispute and avoids the use of subjective or interpretative phrasings. Concrete descriptions have been shown to be low on disputability and high on verifiability (Semin \& Fiedler, 1988). This suggests that language abstraction may be 
used as an instrument that allows communicators to design their messages with their audience in mind.

Several studies on the role of the receiver in the sender's use of language abstraction examined the effects driven by presence of a receiver (Semin, De Montes, \& Valencia, 2003), the identifiability of sender (Douglas \& McGarty, 2001; 2002), and the tie between sender and receiver, such as interdependence (Montes, Semin \& Valencia, 2003), groupmembership (Wigboldus, Semin, \& Spears, 2005), and shared political views (Rubuni \& Sigall, 2002). Consider for instance the study of Semin et al. (2003), who showed that people do not adapt the linguistic abstraction of their messages when they were led to believe that no one would read their description (i.e., that they could keep their description private). Only when they thought that someone would read their description, they systematically used more abstract and concrete language. This following study will examine if consumers adapt their use of language abstraction language to the product attitude of their audience. As a result, an identical product experience may be communicated differently toward different receivers. In fact, it has been argued that this fitting ones product referral to a unique audience is one of the reasons why word of mouth is such a powerful medium (Herr, Kardes, \& Kim, 1991). In parallel with the systematic variations in language abstraction in word of mouth of Chapter 2, I suggest that consumers use more abstract language to describe experiences that are in line with the product attitude of a conversation partner. Thus, communicators will use more abstract language when they describe an experience that is congruent (rather than incongruent) with the receiver's product attitude. I propose to refer to this phenomenon the Receiver Induced Systematic Language Abstraction. Accordingly, it is hypothesized:

H3a Negative product experiences will be communicated more abstractly to receivers with an unfavorable (vs. favorable) product attitude.

H3b Positive product experiences will be communicated more concretely to receivers with an unfavorable (vs. favorable) product attitude. 


\section{Study 3.1: Receiver Induced Systematic Language Abstraction}

This study examined the effect of the product attitude of the receiver on the language use of the sender. The participants were informed about a receiver's attitude toward the product under consideration, and were asked to communicate a product experience to this receiver.

\section{Method}

\section{Design}

One hundred and fifty-four students were randomly assigned to a 2 (product experience: negative vs. positive) $\times 2$ (product attitude of the receiver: favorable vs. unfavorable) between subjects design, with product category (razor blades and yogurt) as within subjects factor. Participants experienced two of the four conditions of the $2 \times 2$ between subjects design, which means one condition for each product. Conditions were counterbalanced, and the two products were shown in random order.

\section{Procedure}

Product experiences were presented by means of film clips of about ten seconds showing either a student shaving (razor blade) or a student tasting yoghurt (yoghurt)(as in Studies 2.2, and 2.3). The clip showed either a positive or negative product experience, depending on the condition. For instance, the yogurt clips showed a student who tastes Fresco yogurt (a fictitious brand). Then, she either smiles, approvingly nods and eats another spoonful (positive experience) or frowns, shows disgust, and pushes the bowl of Fresco yogurt away from her (negative experience).

The product attitude of the receiver was presented in the form of an audio recording in which the receiver expressed his/her product opinion. For instance, for the product Fresco yogurt, the participants would hear a recording in which the receiver said (negative description in between brackets):

\footnotetext{
"Fresco yogurt? I know that yogurt. I tried it once and I really liked [did not like] it"
} 


\section{Dependent Variables}

The participants were exposed to four descriptions of the positive [negative] product experience according to the four levels of the linguistic category model, see Appendix B. As in Chapter 2 Study 2.2, the participants were asked to choose the description that they would use if they were to communicate the product experience to someone else ('Use of Language Abstraction'), and they rated all four descriptions on the extent to which they were an appropriate description of the product experience ("Abstraction Index').

\section{Results}

\section{Use of Language Abstraction}

Data for each product were analyzed in separate ANOVAs, with product experience (negative vs. positive) and receiver's product attitude (favorable vs. unfavorable) as between subject factors and the use of language abstraction as dependent variable. The analyses revealed product experience $\mathrm{x}$ receiver attitude interactions for both products: razor blades $(F(1,151)=47.81, p<.001)$ and yogurt $(F(1,151)=9.66, p<.01)$. All main effects were non-significant, except for the experience effect for razor blades $\left(M_{\text {positive }}=2.88\right.$ vs. $\left.M_{\text {negative }}=2.33, F(1,151)=13.89, p<.001\right)$. Product experiences that correspond to the product attitude of the receiver were communicated more abstractly than experiences that contradict the attitude of the receiver.

In line with Hypothesis 3a, planned contrasts showed that negative experiences were described more concretely to receivers with favorable brand attitudes than to receivers with unfavorable brand attitudes: razor blade $(F(1,151)=$ $28.88, p<.001)$, and yogurt $(F(1,151)=9.12, p<.01)$, see Figure 10a and 10b. Confirming Hypothesis $3 \mathrm{~b}$, the positive experience with the razor blade was described more abstractly to receivers with a favorable brand attitude than to receivers with an unfavorable brand attitude $(F(1,151)=19.27, p<.001)$. For yogurt, a tendency in the correct direction was found, however there was no significant difference $(F(1,151)=$ $1.97, p=.16)$. 
$\square$ Favorable Attitude Receiver $\square$ Unfavorable Attitude Receiver

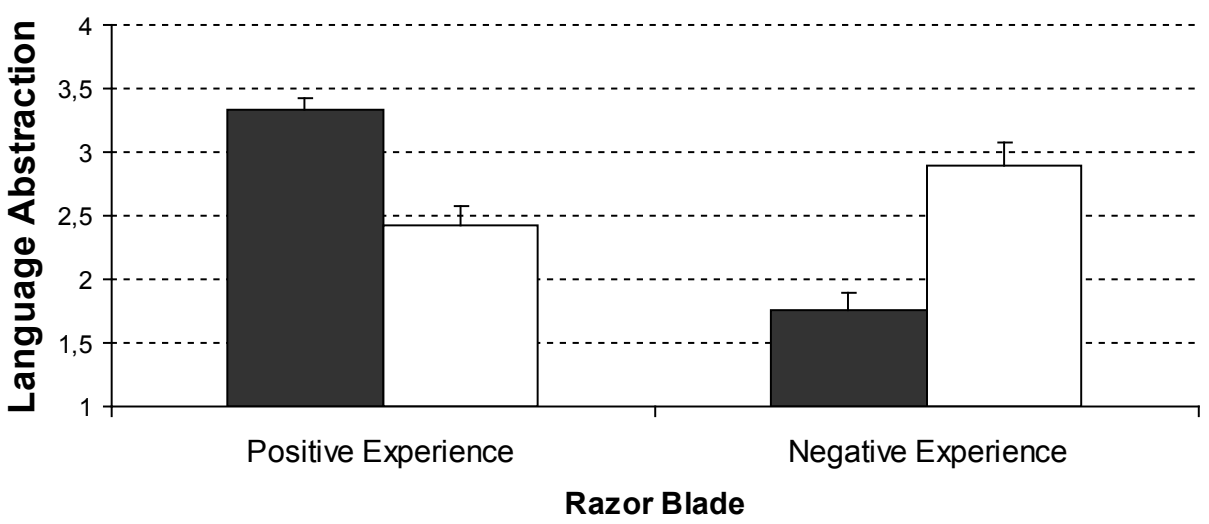

Figure 10a.

Study 3.1. Effect of product experience and receiver product attitude on the Use of Language Abstraction for razor blade: Mean (SE)

$\square$ Favorable Attitude Receiver $\square$ Unfavorable Attitude Receiver

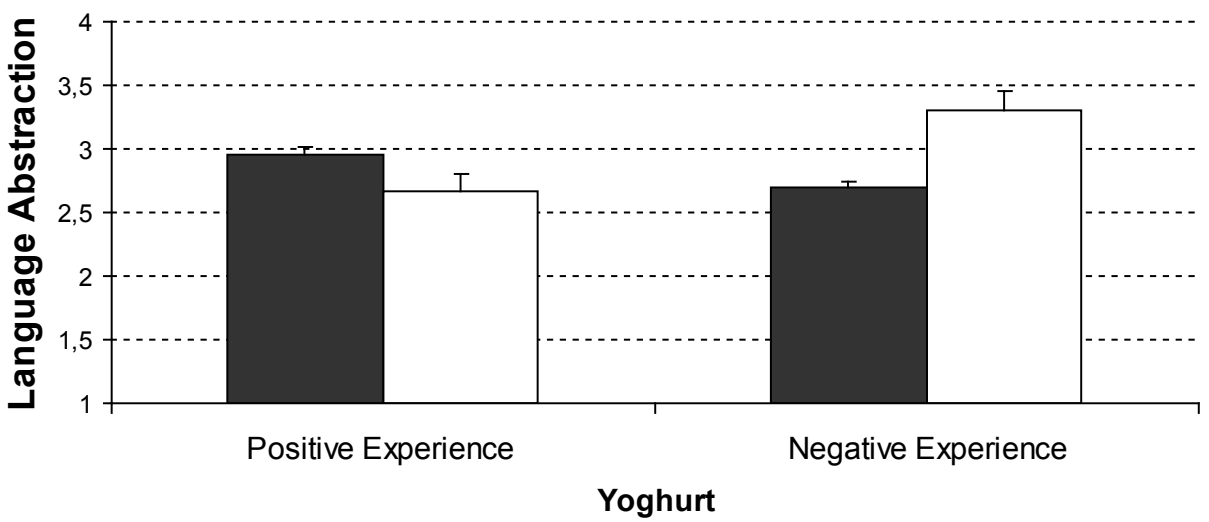

Figure 10b.

Study 3.1. Effect of product experience and receiver product attitude on the Use of Language Abstraction for yoghurt: Mean (SE)

\section{Abstraction Index}

For the abstraction index, similar ANOVAs with product experience and receiver's product attitude revealed the expected interaction effect for both razor blades $(F(1$, $151)=38.50, p<.001)$ and yogurt $(F(1,151)=14.34, p<.001)$, as well as a main effect of 
experience for razor blades $\left(M_{\text {positive }}=2.48\right.$ vs. $\left.M_{\text {negative }}=2.32, F(1,151)=10.61, p<.01\right)$. Product experiences consistent with the product attitude of the receiver were communicated more abstractly than inconsistent experiences. In line with $\mathrm{H} 3 \mathrm{a}$, planned contrasts showed a higher level of language abstraction when negative experiences were communicated to a receiver with a unfavorable (rather than favorable) toward the brand: razor blades $(F(1,151)=14.28, p<.001)$, yogurt $(F(1,151)$ $=3.85, p=.05)$. In line with $\mathrm{H} 3 \mathrm{~b}$, language abstraction was lower when positive experiences were communicated to a receiver with a unfavorable (vs. favorable) attitude brand attitude: razor blades $(F(1,151)=25.17, p<.001)$, and yogurt $(F(1,151)=$ $11.36, p<.01)$, see Figure 11a and 11b.

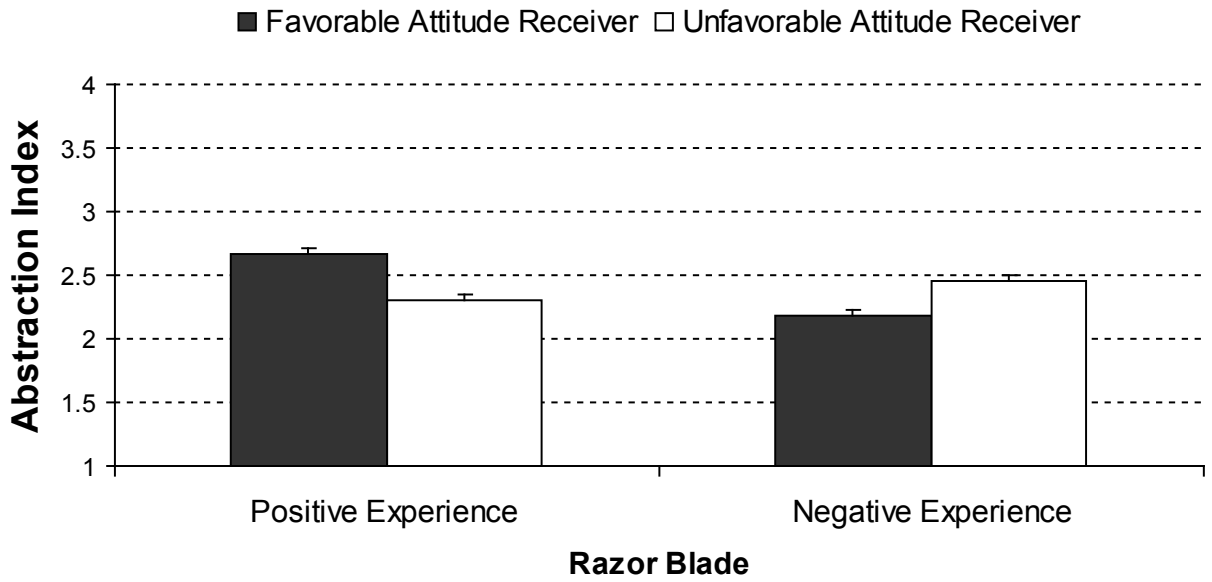

Figure 11a.

Study 3.1. Effect of product experience and receiver product attitude on the Abstraction Index for razor blade: Mean (SE). 
$\square$ Favorable Attitude Receiver $\square$ Unfavorable Attitude Receiver

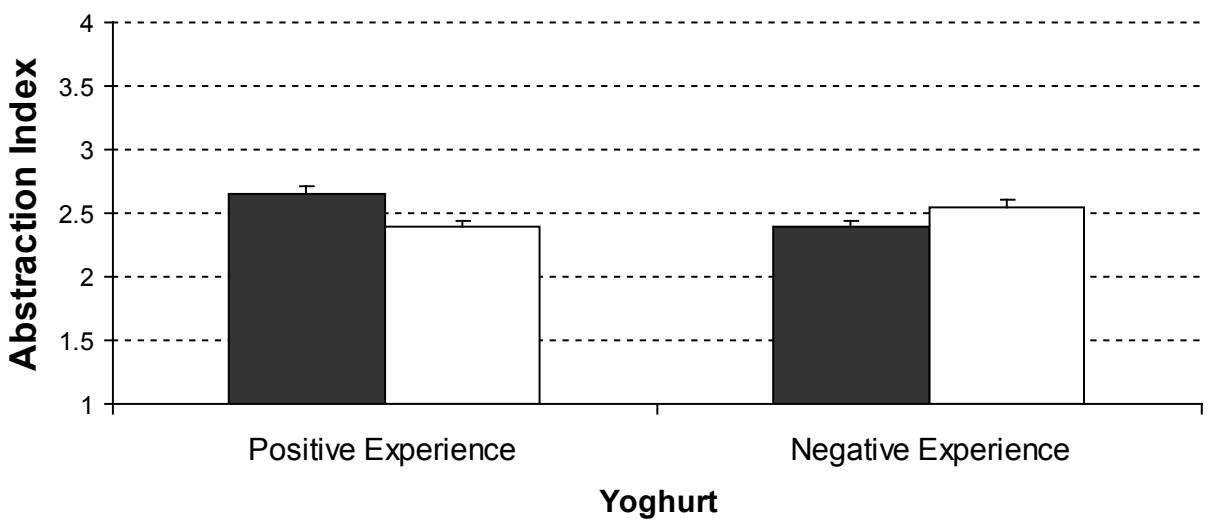

Figure 11b.

Study 3.1. Effect of product experience and receiver product attitude on the Abstraction Index for yoghurt: Mean (SE).

\section{Discussion}

Study 3.1 provides evidence for systematic variations in language abstraction based on the product attitude of the receiver, a phenomenon which was labeled the Receiver Induced Systematic Language Abstraction. Consistent with the hypotheses, consumers who talk about product experiences have been shown to match the level of abstractness of the language they use to the product attitude of the receiver. More specifically, the same positive product experience was communicated in a more abstract manner to a receiver with a favorable brand attitude than to a receiver with an unfavorable brand attitude. Conversely, negative experiences were described in a more concrete manner if the receiver had a favorable attitude compared to an unfavorable attitude. These results support the suggestion that consumers may be implicitly aware of the effects of language abstraction on an audience. Moreover, these findings suggest that language abstraction is used in a strategic manner, in this case to adapt word-of-mouth communication to an audience. 


\subsection{Effect of Communication Goal on Language Abstraction}

Similar to other influence tactics, such as the construction of choice sets (Hamilton, 2003), consumers may modify their use of linguistics to guide the thoughts and feelings of the receiver and create a desired reality. In such, language helps people to achieve their goals. Language can be used to direct the attention of the audience to a particular facet of reality: higher levels of abstraction emphasize the general qualities of the product. Abstractly describing a positive experience highlights the stability of the event and raises the perceived likelihood of recurrence of a positive experience. Thereby one paints a more favorable picture of the product under consideration. Such strategic use of language abstraction was demonstrated by Douglas and Sutton (2003), who asked participants to describe events in a way that would be most (or least) favorable for the actor of the behavior. In response to this instruction, their participants chose more abstract descriptions for positive behaviors and more concrete descriptions for negative behaviors. However, in previous studies the communication goal was focused at the levels of language abstraction of the descriptions (by instructions as "choose the description which frames the information most positive" - Douglas \& Sutton, 2003 Study 1). Thus, the strategic language use depended on explicit instructions which focus the attention of the sender on (the differences in) the language of the four descriptions.

Extending previous research on the effect of communication goals on language abstraction (Douglas \& Sutton, 2003; Semin, et al., 2005; Wenneker et al., 2005), this dissertation will examine the effects of a communication goal focused at the product attitude of a receiver, more specifically a persuasion goal to reach a desired attitude of the receiver. The goal to persuade other consumers of the superiority or inferiority of a product is a frequent motive for word of mouth (Sundaram, Mitra, \& Webster, 1998; Thurau, Gwinner, Walsh, \& Gremler, 2004). It is argued that consumers can adapt language abstraction to communication goals (Wenneker et al., 2005), hence it could also be possible that consumers adapt linguistic abstraction to a desired attitude of the receiver. The study will examine language use in the presence or absence of such persuasion goals, to observe whether consumers can "translate" such a general communication goal into an appropriate level of language abstraction. Consumers who have the goal of persuading others of the quality of a product should aim to generalize positive product experiences and portray the favorable experience as typical and diagnostic for the brand. Thus, they should be more likely to use abstract 
language to describe product experiences. In contrast, these consumers should also be inclined to portray unfavorable product experiences as exception to the rule, and avoid generalizing the information to the brand level. Accordingly, I hypothesize:

H4a Negative product experiences will be communicated more concretely if consumers have the goal of persuading a receiver of the high quality of a product.

H4b Positive product experiences will be communicated more abstractly if consumers have the goal of persuading a receiver of the high quality of a product.

\section{Study 3.2: Effect of Desired Product Attitude on Language}

\section{Abstraction}

This study examined whether consumers employ language abstraction strategically when they communicate with or without the goal to persuade someone of the high quality of the product. Note that the participants of this study have no prior attitudes towards the brands of this study.

\section{Method}

\section{Design}

One hundred fifty-two students were assigned to a mixed design with 2 (product experience: negative vs. positive) x 2 (communication goal: persuasion goal vs. no persuasion goal) $\times 2$ (product category: telecommunication network and dishwasher), where persuasion goal was a between subject variable and product experience and product category were within subjects factors. For both products, each participant was assigned to either a persuasion goal or no persuasion goal. There were different condition combinations between the communication goal and the product experience for the two products - either two positive product experiences for both products, two negative product experiences, or mixed experiences. The conditions were counterbalanced and the products were shown in random order. There was no effect of these combinations on the dependent variables. 


\section{Procedure}

All participants were asked to describe a product experience to someone else. In the persuasion goal condition, participants were additionally told that they had to try to convince this person of the high quality of the product. As in Study 3.1 product experiences were presented by means of film clips. They showed either a student taking a glass from the dish washer or a student calling on a mobile phone (telecommunication network). The clip was either a positive or negative product experience, depending on the condition the participants was in. For instance, the dish washer clips showed a student who finds either a shiny clean glass in the dish washer, approvingly nods and smiles and takes the glass (positive experience) or finds a dirty glass in the dish washer, frowns, sighs, and puts the glass down (negative experience).

Similar to Study 3.1, participants were presented with four descriptions and asked to choose the most relevant description ('Use of Language Abstraction'), and rate all four on appropriateness ('Abstraction Index').

\section{Results}

\section{Use of Language Abstraction}

Data for each product were analyzed in separate ANOVAs, with communication goal (persuasion vs. no activation of persuasion) and product experience (negative vs. positive) as factors, and use of language abstraction as dependent variable. For dishwasher, the analyses revealed main effects of experience $\left(M_{\text {positive }}=3.00 \mathrm{vs}\right.$. $M_{\text {negative }}$ $=2.61),(F(1,148)=8.56, p<.01)$ and of communication goal $\left(M_{\text {persuasion }}=2.66\right.$ vs. $M_{\text {no }}$ persuasion activated $=2.95),(F(1,148)=4.45, p<.05)$. For telecommunication network, a main effect of experience emerged $\left(M_{\text {positive }}=2.96\right.$ vs. $\left.M_{\text {negative }}=2.51\right),(F(1,148)=4.79, p<$ .05). More importantly, the analyses revealed communication goal $x$ product experience interaction effects on choice of description: telecommunication network $(F(1,148)=21.68, p<.001)$, and dishwasher $(F(1,148)=27.99, p<.001)$. In line with Hypothesis $4 \mathrm{a}$, planned contrasts showed that participants chose more concrete descriptions for unfavorable product experiences in the presence of this persuasion goal than in the absence of a persuasion goal: telecommunication network $(F(1,148)=$ 13.01, $p<.001)$, and dishwasher $(F(1,148)=27.38, p<.001)$, see Figure 12a and 12b. Conversely, and in line with Hypothesis $4 \mathrm{~b}$, favorable experiences were described more abstractly when a persuasion goal was present: telecommunication network $(F(1$, 
$148)=8.89, p<.01)$, and dishwasher $(F(1,148)=5.06, p<.05)$. Thus, with a persuasion goal, information that supported the goal was communicated more abstractly, compared to information that opposed the goal.

$\square$ No Persuasion Goal $\square$ Persuasion Goal

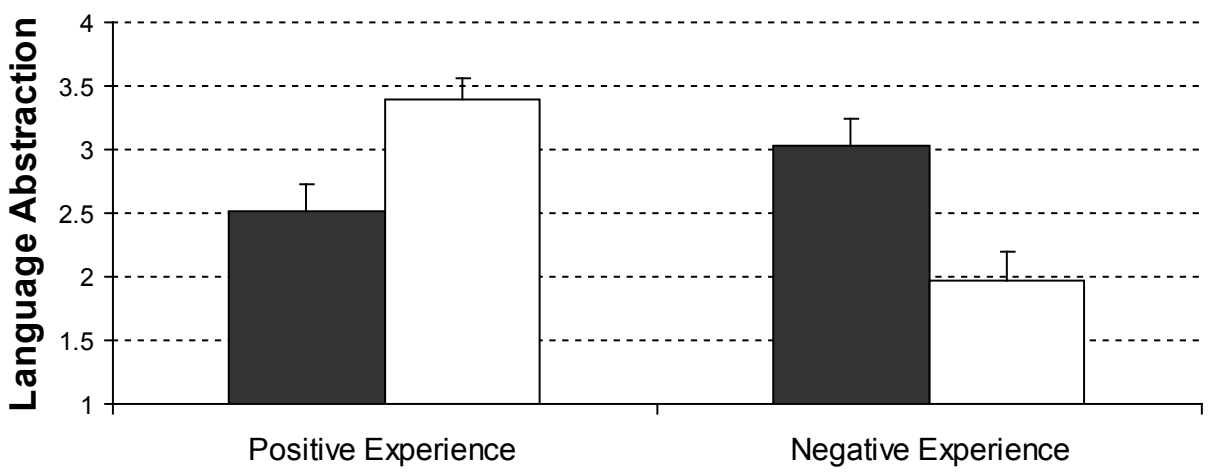

Telecommunication network

\section{Figure 12a.}

Study 3.2. Effect of communication goal and product experience on the Use of Language Abstraction for telecommunication network: Mean (SE).

\section{$\square$ No Persuasion Goal $\square$ Persuasion Goal}

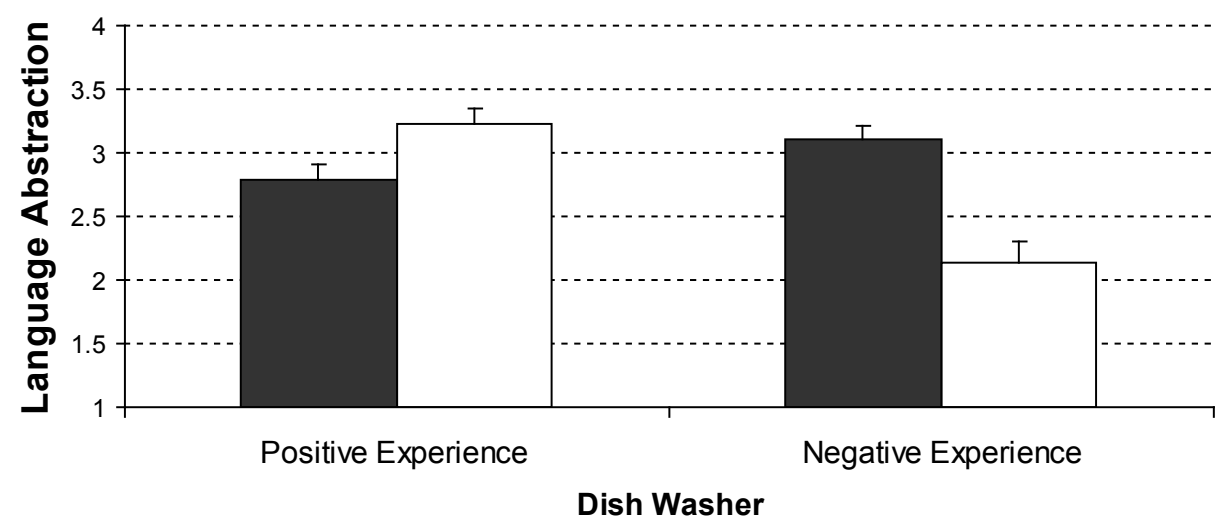

\section{Figure 12b.}

Study 3.2. Effect of communication goal and product experience on the Use of Language Abstraction for dish washer: Mean (SE). 


\section{Abstraction Index}

Similar results were found for the abstraction index: communication goal $\mathrm{x}$ product experience interactions for both products: network $(F(1,148)=17.85, p<.001)$ and dishwasher $(F(1,148)=18.72, p<.001)$. In addition, the analyses revealed main effects of experience for the dishwasher $\left(M_{\text {positive }}=2.64\right.$ vs. $\left.M_{\text {negative }}=2.42\right),(F(1,148)=13.21, p$ $<.001)$ and of communication goal for the dishwasher $\left(M_{\text {persuasion }}=2.46\right.$ vs. $M_{\text {no persuasion }}$ activated $=2.61),(F(1,148)=5.56, p<.05)$. There were no significant main effects for the communication network. In line with Hypothesis $4 \mathrm{a}$, planned contrasts showed that unfavorable experiences were seen as more appropriately described concretely when a persuasion goal was present compared to when this was absent: telecommunication network $(F(1,148)=14.68, p<.001)$, and dishwasher $(F(1,148)=22.34, p<.001)$, see Figure 13a and 13b. Conversely, and in line with Hypothesis H4b, abstract descriptions were seen as more appropriate for positive product experiences in the presence of a persuasion goal than in its absence. This effect was significant for the network $(F(1$, $148)=4.64, p<.05)$, but not for the dishwasher $(F(1,148)=1.94, p=.166)$. Thus, with a persuasion goal, a higher level of abstraction was seen as more appropriate for information that supported (vs. opposed) the goal.

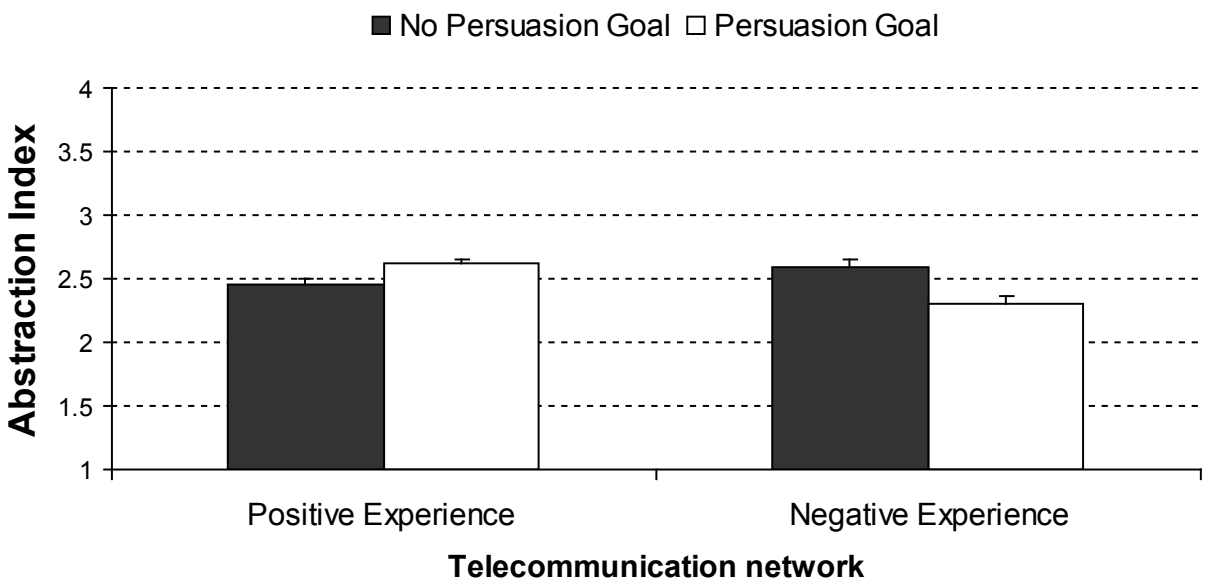

Figure 13a.

Study 3.2. Effect of communication goal and product experience on the Abstraction Index for telecommunication network: Mean (SE). 


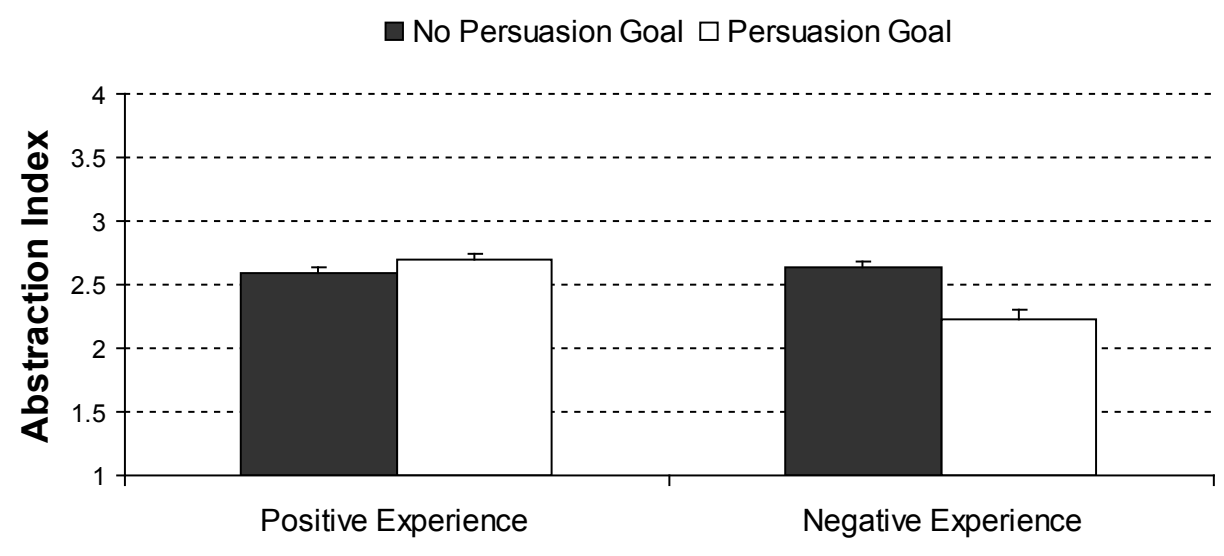

Dish Washer

Figure 13b.

Study 3.2. Effect of communication goal and product experience on the Abstraction Index for dish washer: Mean (SE).

\section{Discussion}

An important goal of word-of-mouth communication is to persuade other consumers of the superiority or inferiority of a product on one or more dimensions. This study showed the strategic use of language abstraction with a general communication goal. Even when the sender does not have a priori product attitude, a persuasion goal leads to strategically use language abstraction. In the presence of a goal to attain a positive attitude from a receiver, positive product information is described more abstractly, and negative information is described more concretely. The results of this study show how senders strategically use language abstraction to achieve specific communication objectives, such as the goal to persuade.

\subsection{General Discussion}

The previous chapter introduced how consumers adjust their use of language abstraction to their a priori product attitudes. The present chapter added to these findings by exploring whether consumers also use language abstraction strategically. It examined the adaptive nature of consumers' use of language abstraction to two important aspects of the sender-receiver dyad: the receiver's actual attitude and the 
receiver's desired attitude. It was hypothesized that the language that consumers use to describe their product experiences is influenced by the attitudes of their audience, and by persuasion goals aimed at receivers' product attitude.

The first main finding of this chapter is that consumers' language use is responsive to their audience. A phenomenon referred to as the Receiver Induced Systematic Language Abstraction, in which language abstraction is adapted to the brand attitude of the audience. More abstract language is used for product information that is consistent to the product attitude of a receiver compared to inconsistent product information. More specifically, favorable product experiences were found to be communicated more abstractly to receivers with a positive brand attitude than to receivers with a negative attitude. Unfavorable experiences were described more concretely to receivers with positive brand attitudes than to receivers with negative attitudes. Although communicators have long been known to adapt their messages to fit with their audience (e.g., Fussell \& Kraus, 1992; Higgins \& Rholes, 1978), the role of receivers has received limited attention in the study of linguistic abstraction. Future research could extend this work by incorporating other aspects of the receiver, including product expertise or involvement. When a non expert discusses a product with an expert, this person may avoid using abstract language, because the large knowledge and experience of the expert on the topic makes the non expert feel that he has no ground to generalize the information of one single experience to the brand or product in general. One may also stick to a more concrete framing to avoid dispute and counter arguing which is more likely activated by abstract language, since an expert will more likely win a discussion about the topic in question.

The second main finding is that consumers use language abstraction to achieve a specific effect on their audience. Study 3.2 showed how language use is strategically matched to the specific goal with which consumers communicate to reach a desired effect on the product attitude of a receiver. Consumers who had the goal of persuading someone used more abstract language when they communicated information that supported this goal and concrete language if the information countered this goal. No such effects were found without a persuasion goal. Even though persuasion is an important goal for word of mouth, particularly in the marketing and scales context, future research may examine how language abstraction is influenced by other goals such as the goal to signal expertise or strengthen interpersonal relations. For instance, a goal to self protect will probably activate the 
use of more concrete language, while a self enhancement goal may trigger more abstract word of mouth.

For future research it would be interesting to examine what would happen to language abstraction when a consumer tries to convince a negative receiver of a positive product opinion, or when the sender has a negative opinion about a product, but the receiver is positive. A naïve prediction could be that combinations between the product attitudes of the sender and the receiver with positive and negative product information can lead to extremely concrete or extremely abstract messages. For instance, when two conversation partners are both very fond of a brand, they will probably both use very abstract language when sharing positive word of mouth about experiences with this brand, and very concrete language in case of positive word of mouth. This may be a subtle way to dissociate oneself from out-groups (consumers who dislike this brand)(White \& Dahl, 2007). Likewise, when a consumer has a unfavorable product attitude and is talking to another consumer who is part of the same reference group, then the positive qualities of the product will probably be discussed very concretely, while for the negative qualities enhanced language abstractness might be expected.

This latter prediction is in line with the study of Rubini and Sigall (2002) who proposed a higher level of linguistic abstractness when presenting one's political view to an agreeing (vs. mixed) receiver. But my prediction seems to contrast Wigboldus, Spears, and Semin (2005), who argued that the systematic linguistic bias will not be activated when the sender, receiver, and described actor belong to the same reference group. More specifically, they found that language use is not systematically varied in the descriptions of expected and unexpected actions of a female actor described by a female speaker to a female receiver. Wigboldus proposed that variations in language abstraction only occur if stereotypes are activated, for example by social categorizations which create a comparative context (e.g., females vs. males)(Oakes, 1987; Tajfel \& Turner, 1986). When such a social comparative context is not made salient, such as in the case of a female talking to a female about the behavior of another female, there is no reason for systematic biases in language abstraction. The difference between Wigboldus' findings and word of mouth is however that consumers' a priori product attitudes automatically incite a comparative context for their product experiences, and that consumers compare each product experience to their (positive or negative) product attitude. Thus, in case consumers have an a priori attitude toward a 
product, there will be a basis for systematic variations in language abstraction, regardless of the attitude of the receiver.

In cases where the sender and receiver have different product attitudes, my predictions will strongly depend on the specific communication situation. Although people can be egocentric beings who find it inherent difficult to detach themselves from one's own perspective (Epley \& Caruso, 2008; Kruger, Epley, Parker, \& Ng, 2005; Ross \& Sicoly, 1979), there should be many situations where receivers and their attitudes are used as a frame of reference, even when they are in conflict with the sender's own opinions. For instance, the abstractness of the speaker's message may be strongly affected by the receiver's product attitude when the receiver has more power than the speaker, or when a positive relationship with the receiver is highly important. 



\section{Chapter 4. Effects of Language Abstraction on Receivers}

\subsection{How does Language Abstraction Affect Receivers?}

Previous research on language abstraction mainly focused on the factors affecting the sender's use of language abstraction. Far less is known about the ways in which receivers respond to communicators' use of abstract versus concrete language. In this dissertation, it is argued that language can be used as a communication signal. Above and beyond the actual content and valence of a message, receivers may be able to pick up on subtle cues of language use, and are affected by it in their behavioral intentions. The findings of Chapter 3 also suggest that senders take the effects of language abstraction on receivers into account when describing product experiences. This chapter examines the effects of language abstraction on receivers of word of mouth.

Research on language abstraction showed that people are able to infer from the language abstraction of a message how the message was intentionally framed (Douglas \& Sutton, 2006). In that study, participants were explicitly informed that they would be given descriptions about (positive and negative) behaviors of a person, and that these descriptions had been written with the intent to present the events in either a positive or negative manner. The results indicated that participants could use the level of language abstraction of the descriptions to infer whether the author had intended to paint a positive or negative picture of the actor. The present chapter first sought to establish if consumers can also infer the product attitude of the sender of a word of mouth message from the language abstraction of the message.

Note that inferring a sender's product attitude from the language used in word of mouth is a complicated task. Consumers may have a multitude of motives for word of mouth (Sundaram, Mitra, \& Webster, 1998) which could affect the content and evaluative tone of their communication as well as the language used. Receivers therefore have many possible factors to which they can attribute the use of concrete or abstract language in word of mouth.

Taking the effectiveness of language abstraction one step further: how does language abstraction affect receivers? Is an abstract or concrete positive product 
referral more persuasive? And when can one or the other be more persuasive? In line with the linguistic category model, abstract descriptions of behaviors have been found to lead to more dispositional inferences about the actor (Wigboldus, Semin, \& Spears, 2006). Wigboldus and colleagues studied receivers' processing of stories that were either consistent or inconsistent with their stereotypes of the actors in the story. They found that stereotype-consistent stories led to more dispositional inferences about the actor, and that this effect was mediated by language abstraction. But will more abstract word-of-mouth messages also have a stronger effect on receiving consumers? An impressive body of marketing research has shown the persuasive effect of concrete and vivid information (Kim, Kardes, \& Herr, 1991; Kisielius \& Sternthal, 1984). This seems to contradict the effects of Wigboldus and colleagues. This chapter will examine the actual effect of language abstraction on receiver's behavioral intentions. Additionally, I will incorporate the fact that consumers are not passive receivers, they have intentions and objectives they are considering when receiving new product information. The last study of this chapter reveals how a consumers' mindset moderates the impact of language abstraction on receivers.

This chapter of the dissertation extends previous findings by examining the effect of language abstraction of a product message on receiving customers. Study 4.1 will show whether consumers are able to infer someone's product attitude from the level of language abstraction that they use to describe positive and negative product experiences. Study 4.2 demonstrates the effects of the language abstraction of word of mouth on the receiver's buying intention, and Study 4.3 replicates these findings when correcting for valence. Study 4.4 introduces the consumer's mindset as a moderator for the persuasiveness of language abstraction.

\subsection{Effect of Language Abstraction on Inference of Product Attitude of Sender}

Speakers use language to convey a particular meaning, and anticipate that receivers recognize their communication intent. Receivers are oriented towards recovering the sender's intention (Sperber \& Wilson, 1995). They go beyond the information given and examine the specific linguistics employed to construct the message meaning. Based on the findings of Douglas and Sutton (2006), I argue that language abstraction can be used to infer a sender's product attitude. If consumers are able to infer someone's product attitude from the level of language abstraction of a word-of-mouth 
message, this assumes that consumers are (at least implicitly) aware of the fact that communicators use more abstract language when they describe experiences that are consistent with their expectations. This would lead recipients to infer that communicators have a more positive product attitude when they describe a positive experience in an abstract rather than concrete manner. In descriptions of negative experiences, the use of more abstract language should lead receivers to infer that the sender has a less favorable product attitude. In previous work (Douglas \& Sutton, 2006), participants were shown a picture of person acting (positive or negative behaviors) and were asked to match descriptions of this behavior to a role of the sender (who was either a friend, enemy or neutral observer of the actor). In the present study, as in every day life, participants did not receive this insight. They were unaware of the actual experience, and were not aware of the role of the sender. Moreover, the dynamic and interactive elements in word of mouth and the multiple motives for product referrals may make inferring a sender's product attitude a challenging task.

H5a When negative product experiences are described more abstractly (vs. concretely), consumers will infer that a sender has a less favorable attitude toward the product.

H5b When positive product experiences are described more abstractly (vs. concretely), consumers will infer that a sender has a more favorable attitude toward the product.

\section{Study 4.1: Effect of Language Abstraction on Inferred Product Attitudes held by Senders}

This study examined the effect of language abstraction on the receiver's inferences about the sender's opinion about the product. The participants received either a positive or negative product referral that was presented in the form of word of mouth from another consumer. For replication purposes four different product categories were used in this study (computer, car, razor blade, and dessert). For each product four descriptions were created which correspond to the four different levels of language abstraction proposed by the model (Semin \& Fiedler, 1988)(see Appendix C for the product statements for each of the four products). For instance, for the product 
razor blade the four descriptions were respectively from concrete to abstract (DAV, IAV, SV, and Adj)(negative experiences within brackets):

I shave with Shaft razor blades [the Shaft razor blades do not glide well]

I use Shaft razor blades [the Shaft razor blades do not function well]

I [do not] like Shaft razor blades

Shaft [does not have] has nice razorblades

\section{Pretest}

In a pretest, the language abstraction of the sets of descriptions for the four products of this study was recorded. Ninety-seven participants from the same subject pool received a general explanation about the concepts 'concrete' and 'abstract descriptions'. For the four products, the participants were shown a set of the four descriptions of a positive product experience at a random order, and were asked to indicate the abstractness per description from (1) concrete to (7) abstract. Participants were anticipated to observe and indicate the increasing language abstraction from DAV (most concrete) to IAV to SV to Adj (most abstract). A repeated measures ANOVA on the perceived language abstraction with language abstraction of the description and product as within subject variables indicated a significant effect of language abstraction $(F(3,864)=104.29, p<.001)$ and an interaction effect $(F(9,864)=$ $4.92, p<.001)$. Follow-up planned comparisons revealed that the DAV descriptions (2.40) was rated as more concrete than the IAV descriptions $(3.33, F(1,96)=75.29, p<$ $.001)$, which in turn were rated as more concrete than the SV descriptions $(4.88, F(1,96)$ $=56.01, p<.001)$, which in turn were rated as more concrete than the Adj descriptions $(5.41, F(1,96)=8.34, p<.01)$. Repeated measures ANOVA's per product indicated that language abstraction had a significant effect on the observed abstraction for all products (razor blade: $\mathrm{F}(3,288)=89.49$, $\mathrm{p}<.001$, dessert: $\mathrm{F}(3,288)=30.52$, $\mathrm{p}<.001$, computer: $F(3,288)=51.48, p<.001$, and car: $F(3,288)=65.10, p<.001)$. For each product, follow-up planned comparisons revealed that all the differences between the four levels were in the indicated direction for all products (all $F^{\prime} s>4.4, p^{\prime} s<.05$ ), except the difference between the SV and Adj descriptions of the computer $(\mathrm{F}<1)$. 


\section{Method}

\section{Design}

Two hundred and twelve students were randomly assigned to a counterbalanced design with 2 (type of word of mouth: negative vs. positive) x 4 (language abstraction: DAV vs. IAV vs. SV vs. Adj) x 4 (product category: computer, car, razor blade, and dessert). Word-of-mouth type was a between subjects variable and the latter two were combined within subjects variables. For this study, a design was constructed in which a single participant received only one word-of-mouth statement per product, and only one instance of each of the four levels of language abstraction. Pairings of products and language abstraction were randomized. Thus, a particular participant could receive a statement about a car brand that was presented as an IAV, a statement about a computer brand presented as a DAV, a statement about a razor blade brand as an SV, and a statement about a dessert presented at the Adjective level. Product order was random.

\section{Procedure}

The product statements were introduced by a short scenario which explained that the respondents would receive statements about several products, which had been made by other consumers. After each description, participants were asked to indicate their evaluation of the product attitude held by the speaker. This was done on three ninepoint items: I think the speaker is: very negative (1) / very positive (9) about the product, ...finds the product very bad (1) / very good (9), ...finds the product very unattractive (1) / very attractive (9).

In a post-test, ratings for the valence of each statement (from (1) negative to (5) positive; Maass et al., 1989) were collected, and entered as covariate in follow-up analyses. These analyses will be discussed at the end of the results section.

\section{Results}

\section{Inferred Product Attitudes}

For each type of statement and product, the three scales used to measure respondents' inferences about the product attitude of the sender were combined into one scale. Cronbach's alpha for these indices ranged from .80 to .98 . The data were analyzed with a mixed linear model with type of statements (positive vs. negative) as between subjects variable, language abstraction (DAV vs. IAV vs. SV vs. Adj) as a within 
subjects variable, product category (computer, car, razor blade, and dessert) as a covariate, and subject as a random factor. This revealed an effect of type of statement $(F(1,700)=2384.22, p<.001)^{2}$, of language abstraction $(F(3,407)=16.49, p<.001)$, of product $(F(1,446)=8.87, p<.01)$, and a type of statement $x$ language abstraction interaction effect $(F(3,402)=68.84, p<.001)$. These findings will be explained per type of statement.

\section{Inferences based on Negative Word of Mouth}

For negative word of mouth, a repeated measures ANCOVA on the inferred product attitudes with language abstraction as within subjects factor, product category as a covariate, and subject as a random factor revealed a significant effect of language abstraction $(F(3,252)=11.05, p<.001)$, of product category $(F(3,252)=18.59, p<.001)$ and of subject $(F(3,252)=1.61, p<.01)$. Confirming Hypothesis $5 \mathrm{a}$, an increase in language abstraction in negative word of mouth led receivers to infer a less favorable product attitude for the sender, see Figure 14. More specifically, when a negative experience was described in action verbs as DAV (3.40) and IAV (3.04) than participants inferred the product attitude of the sender as more favorable compared to descriptions using State Verbs (2.74) and Adjectives (2.62). Thus, if a speaker communicates a negative product experience at a more abstract level, the listener infers a less favorable attitude.

\section{Inferences based on Positive Word of Mouth}

A repeated measures ANCOVA of the favorable statements with language abstraction (LCM: DAV vs. IAV vs. SV vs. Adj) as within subjects factor, product category (computer, car, razor blade, and dessert) as a covariate, and subject as a random factor revealed a significant effect of language abstraction on inferred product attitude $(F(3$, $369)=115.07, p<.001)$. It also showed effects of product category $(F(3,369)=51.43, p<$ $.001)$ and subject $(F(3,369)=1.81, p<.001)$, which were not germane to the study. In line with Hypothesis $5 b$, an increase in language abstraction in positive word of mouth led receivers to infer a more favorable product attitude for the sender. Specifically, positive experiences described with DAVs (5.91) or IAVs (5.85) led participants to infer that senders held less favorable product attitudes than product experiences described with SVs (6.87), while Adjectives (7.84) led to the most favorable inferred product ${ }^{2}$ Note that the estimated degrees of freedom are reported for the mixed linear models in study 4.1 and
4.2 , and for sake of clarity the decimals are not reported. 
attitudes (see Figure 14). Thus, if a speaker communicates a positive product experience at a more concrete level, the listener infers a less favorable attitude.

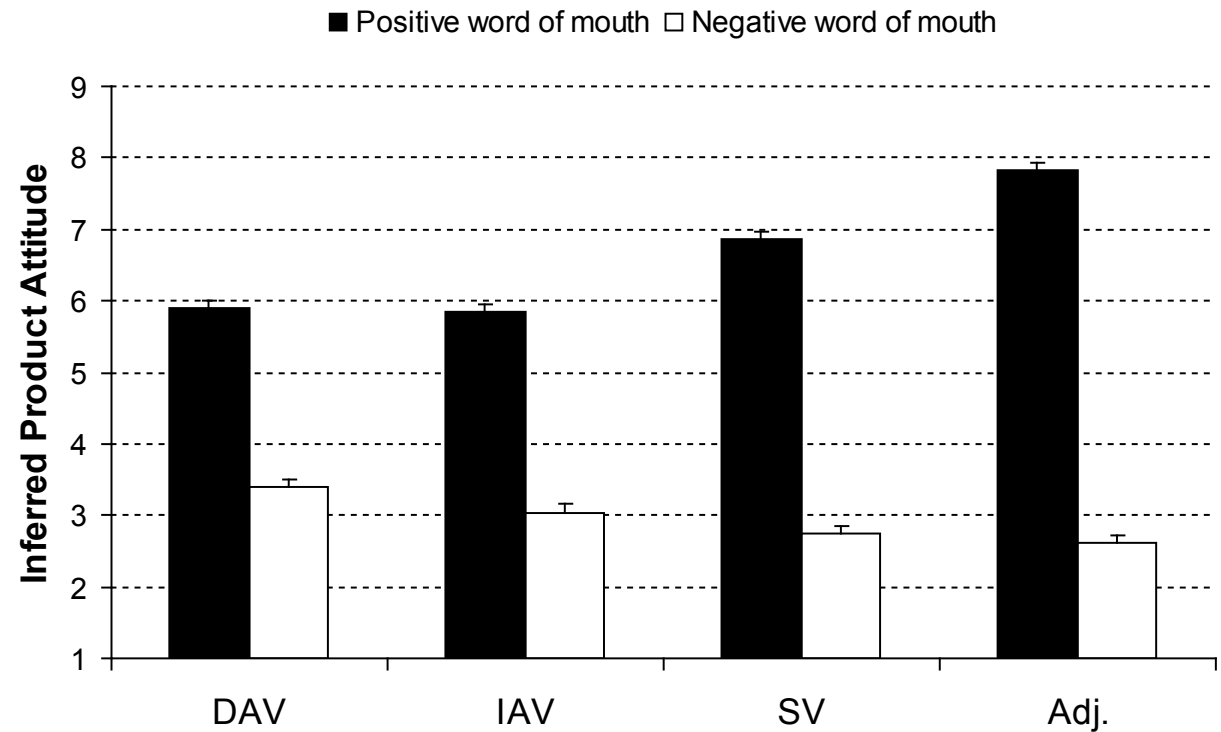

Figure 14.

Study 4.1. Effect of word of mouth (positive vs. negative) and language abstraction (from concrete to abstract: DAV, IAV, SV, Adj) on inferred product attitude of sender: Mean (SE)

\section{Additional Analyses with Valence as Covariate}

Analogously to studies reported in Chapter 2, I examined whether these effects are driven by differences in the valence of the statements. To this end, a post-hoc study was conducted in which one hundred and eighty-two students rated the valence of the favorable and unfavorable descriptions used in this study from negative (1) to positive (5). The mean valence rating for each statement was included as a covariate in the analyses. The results indicated that the effects were not mediated by the valence of statements, and that the inclusion of the valence covariate did not lead to substantial changes in the findings.

For negative word of mouth, a repeated measures ANCOVA with language abstraction as within subjects factor, product category as a covariate, subject as a random factor, and the addition of the rated valence scores as a covariate showed that the rated valence had an effect on the inferred product attitudes $(F(3,251)=15.30, p<$ $.001)$, but the effect of language abstraction on inferred product attitudes remained 
significant $(F(3,251)=8.15, p<.001)$. Thus, after correction for valence, the pattern of means still indicated that an increase in language abstraction leads receivers to infer a less favorable product attitude for the sender $(3.27,3.10,2.63,2.79)$.

For positive word of mouth, the same repeated measures ANCOVA for positive referrals revealed only a marginal effect of the rated valence covariate $(F(1,368)=$ 3.052, $p=.081)$. The effect of language abstraction on inferred product attitude remained significant after entering the covariate $(F(3,368)=6.17, p<.001)$. After correction for valence, the pattern of means still indicated that an increase in language abstraction leads receivers to infer a more favorable product attitude for the sender $(6.10,6.08,6.83$, and 7.46$)$.

\section{Discussion}

This study shows that the use of more abstract language in word-of-mouth communication makes receivers infer that the sender of the word of mouth has a more 'extreme' product attitude. More precisely, abstract (vs. concrete) negative word of mouth leads receivers to infer that the sender has a less favorable product attitude. While for positive word of mouth, I find the reverse: receivers infer that the sender of word of mouth has a more favorable product attitude, if the sender uses more abstract (vs. concrete) language in their word-of-mouth referral. Ergo, abstract language seems to suggest more strong or outspoken opinions. Additionally, these effects remain significant after correcting for the influence of differences in valence between concrete and abstract descriptions.

\subsection{Effect of Language Abstraction on Behavioral Intention}

A logic and interesting next step is studying the persuasiveness of language abstraction. What would be the effect of language abstraction on receivers' attitudes and purchase intentions? Previous research (Wigboldus et al., 2000; 2006) has shown that abstract descriptions of behaviors lead to more dispositional inferences about the actor of the behavior. If abstract language leads to more dispositional inferences, then abstract descriptions of product experiences might also lead consumers to make more or stronger inferences at the level of the brand or product. If the described experience is positive, abstract descriptions should therefore result in higher purchase intentions than concrete descriptions. On the other hand, if the described experience is negative, 
abstract descriptions should result in lower purchase intentions than concrete descriptions.

It is reasonable to expect results in line with Wigboldus et al. (2000) and Douglas and Sutton's (2006) findings, such that abstract word of mouth has a stronger effect on receivers and receivers' inferences of a sender's product attitude compared to concrete word of mouth. Note, however, that it is not self-evident that abstract word of mouth is more persuasive than concrete word of mouth. Building on Semin and Fiedler's (1988) linguistic category model, it is also possible to arrive at an opposite prediction for the effect of language abstraction on the persuasiveness of messages. First, abstract descriptions of an event are less verifiable than concrete descriptions because they go beyond the particulars of the behavior or event. Therefore, abstract descriptions are more open to dispute. Second, concrete descriptions are often more vivid than abstract ones, and vividness has been shown to have persuasive effects (Kim, Kardes, \& Herr, 1991; Kisielius \& Sternthal, 1984). These differences in vividness, verifiability and disputability may reduce the persuasiveness of messages that are presented in abstract language. To gain insight into the persuasive effect of language abstraction on receivers, Study 4.2 examines the impact of language abstraction on the purchase intentions of receivers and will allow us to distinguish between these two competing hypotheses.

\section{Study 4.2: Effects of Language Abstraction on Buying Intention}

This study was designed to provide the first test of the effect of variations in language abstraction on the receivers' buying intentions for the described product. Participants received four word-of-mouth statements pertaining to products from four different categories (i.e., mortgage broker, TV program, club, and detergent) and were asked to indicate their purchase intention for each of these products.

\section{Pretest}

As in Study 4.1, perceived language abstraction of the product statements was pretested among 44 students. Mauchly's test indicated that the assumption of sphericity had been violated for some of the products, I therefore report the corrected degrees of freedom (rounded to whole numbers) using Greenhouse-Geisser. For each product, a repeated measures ANOVA indicated that perceived language abstraction differed significantly between the four levels of the descriptions (detergent: $F(3,108)=$ 
38.72, $p<.001$, mortgage broker: $F(2,95)=20.72, p<.001$, TV program: $F(2,88)=30.70$, $p<.001$, and club: $F(2,92)=49.28, p<.001)$. Follow-up planned contrasts revealed that all the differences between the four levels were significant and in the intended direction for all products $(F S>5.66, p<.05)$, except the difference between the SV and Adj descriptions of the mortgage broker, which was in the right direction but not significant. See Appendix C for the scenarios, product statements, and ratings of perceived language abstraction.

\section{Method}

\section{Design and Procedure}

One hundred fifty-five students were randomly assigned to a 2 (word of mouth: negative vs. positive) x 4 (language abstraction: DAV vs. IAV vs. SV vs. Adj) x 4 (product category: detergent, mortgage broker, TV program, and club) counterbalanced design. Word-of-mouth type was manipulated between subjects, and the other two variables were manipulated within subjects. Similar to Study 4.1, participants received four product statements at different levels of language abstraction for four products. Product order was randomized. Respondents received either a set of positive or negative word-of-mouth statements, preceded by a short scenario that introduced them.

\section{Dependent Variables}

After each scenario and product statement participants were asked how likely they would be to buy this product (or use this service) on a 11-point Juster scale for purchase likelihood (Juster 1966; Wright \& MacCrae, 2007). At the end of the study, participants rated the valence of the statements they had received on a five point scale from negative (1) to positive (5). These ratings allowed us to assess the influence of any possible differences in the valence of the statements.

\section{Results}

A mixed linear model was used with word-of-mouth type (negative vs. positive) as between subjects variable, language abstraction (DAV vs. IAV vs. SV vs. Adj) as a within subjects variable, subject as a random factor, and rated valence of the statement as a covariate on the indicated purchase intention. This analysis revealed main effects of word-of-mouth type $(F(1,593)=5.25, p<.05)$ and rated valence $(F(1,598)=67.48$, 
$p<.001)$, as well as a rated valence $\mathrm{x}$ type of statement interaction $(F(1,598)=7.32, p<$ $.01)$. More important for the hypotheses, a significant language abstraction $x$ type of statement interaction $(F(1,304)=4.02, p<.01)$ was also obtained. For negative word of mouth, an increase in abstraction leads to a decrease in buying intention $(F(4,76)=$ 490.47, $p<.001$; means across products from concrete to abstract corrected for rated valence: $4.57,4.30,3.49,3.47)$. Note that the reported means are corrected for valence of the product statements. For positive word of mouth, the opposite effect was found: an increase in abstraction leads to an increase in buying intention $(F(4,77)=500.17, p<$ .001; means across products from concrete to abstract corrected for rated valence: 4.64, $5.09,6.22,6.18)$, see Figure 15. The means per product are presented in Table 1.

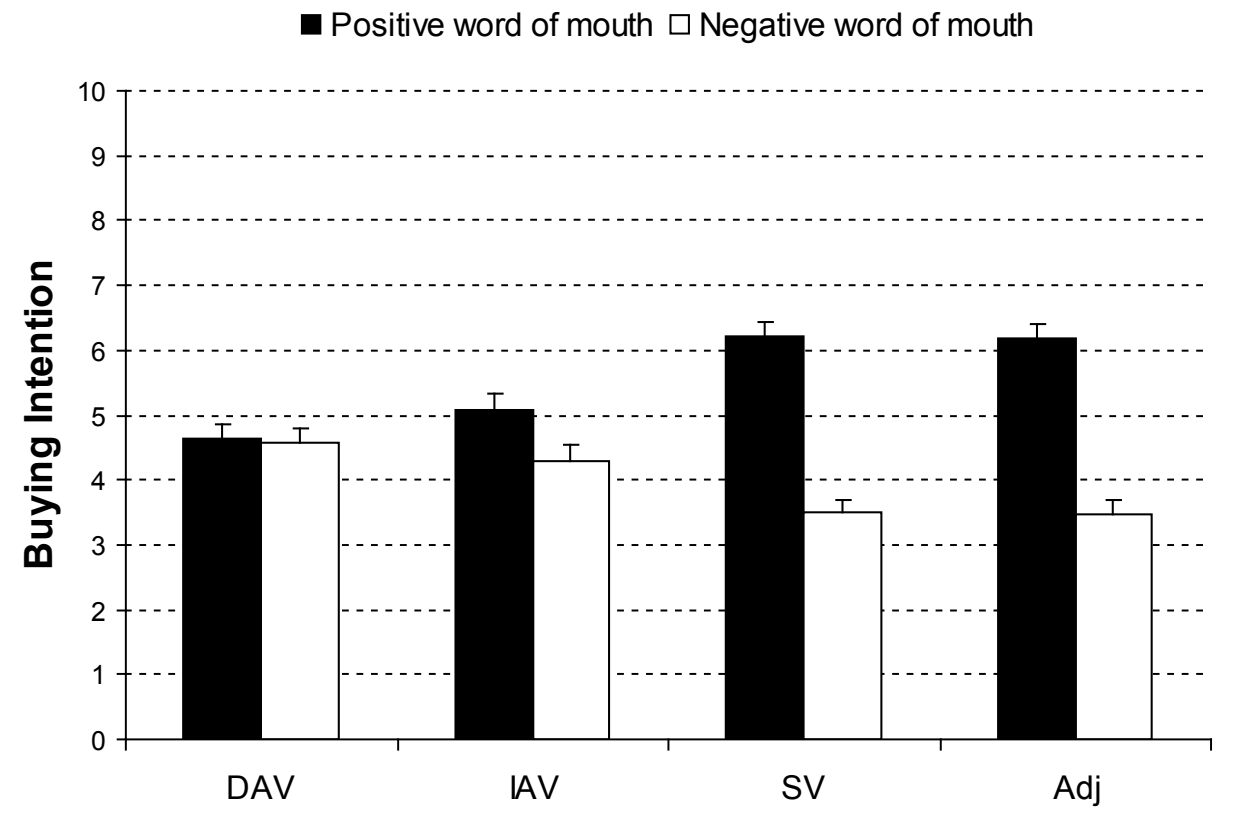

Figure 15.

Study 4.2. Effect of word of mouth (positive vs. negative) and language abstraction on buying intention (from concrete to abstract: DAV, IAV, SV, Adj): Mean (SE) 
Table 1

Study 4.2: Effect of word of mouth (positive vs. negative) and Language Abstraction on Buying Intentions: Mean (SD)

\begin{tabular}{lrlll}
\hline & Detergent & $\begin{array}{l}\text { Mortgage } \\
\text { broker }\end{array}$ & TV program & Club \\
\multicolumn{2}{l}{ Positive experiences } & & & \\
DAV & $5.15(2.03)$ & $5.53(1.65)$ & $5.53(2.34)$ & $2.45(0.94)$ \\
IAV & $4.80(1.58)$ & $7.05(1.57)$ & $5.53(2.34)$ & $2.89(1.20)$ \\
SV & $7.21(1.69)$ & $6.95(1.47)$ & $6.95(1.88)$ & $3.68(2.11)$ \\
Adj & $7.68(1.73)$ & $5.89(1.85)$ & $6.70(1.49)$ & $4.50(1.96)$ \\
& & & & \\
Negative experiences & & & \\
DAV & $5.00(1.59)$ & $4.30(1.22)$ & $4.63(1.64)$ & $4.33(2.17)$ \\
IAV & $4.95(1.39)$ & $2.95(1.22)$ & $4.94(1.63)$ & $4.35(2.39)$ \\
SV & $4.05(1.08)$ & $3.28(1.32)$ & $3.30(1.34)$ & $3.35(1.39)$ \\
Adj & $3.89(1.28)$ & $3.05(1.54)$ & $3.55(1.36)$ & $3.42(1.68)$ \\
\hline
\end{tabular}

\section{Discussion}

This study revealed the persuasive effect of language abstraction on receivers buying intention. Positive word of mouth led to higher purchase intentions when more abstract language was used. For negative word of mouth, more abstract language led to lower purchase intentions. To the best of my knowledge, this provides the first evidence of an effect of language abstraction on receivers' opinions about the described object (in this case: buying intentions).

In Study 4.2, I tried to rule out effects of variations in statement valence by using participants' ratings of statement valence as a covariate in my analyses. To provide additional evidence for the claim that the effects do not result from variations in statement valence, an additional study was run which replicates the results of Study 4.2 with a set of descriptions that was carefully constructed to show no variation on statement valence. 


\section{Study 4.3: Effects of Language Abstraction on Buying Intention and Attitude: Controlling for Valence}

This study examined the impact of language abstraction on the purchase intentions of receivers, when the valence of the stimulus material is carefully held constant between the different conditions. Four word-of-mouth statements about the product ice cream were constructed to represent the different levels of language abstraction according to the linguistic category model, but at the same time remain equal in valence:

I ate the entire cup of Moccalicious ice cream

I entirely finished the cup of Moccalicious ice cream

I liked the Moccalicious ice cream

Moccalicious ice cream is tasty

\section{Pretests}

\section{Pretest 1}

A first pretest $(\mathrm{N}=44)$ with a similar procedure to that used in Study 4.1 and 4.2 on the perceived language abstraction, revealed significant differences in perceived language abstraction $(F(1,129)=39.17, p<.001)$. Planned contrasts showed that the first description (DAV: 2.09) was rated as more concrete than the second description (IAV: 3.27, $F(1,43)=46.73, p<.001$ ), which in turn was rated as more concrete than the third description $(\mathrm{SV}: 4.93, F(1,43)=16.38, p<.001)$, which in turn was rated as more concrete than the last description (Adj: $5.75, F(1,43)=5.90, p<.05)$.

\section{Pretest 2}

Importantly, a second pretest $(\mathrm{N}=68)$ examined the valence of the four descriptions (between subject intermixed with filler statements about other products). An ANOVA with language abstraction as a between subject variable on the valence of the product descriptions showed no variation in the rated valence of the four descriptions $(F(3,64)$ $<1, p=.414)$. This means that the four statements about the ice cream are equal in valence. 


\section{Method}

Seventy-five students received positive word of mouth for a new brand of ice cream from a fellow student. After receiving a product statement, participants were asked how likely they would be to buy the ice cream, using the same 11-point Juster scale as in Study 4.2. In addition, participants indicated their attitude toward the ice-cream on a three-item measure as in Study 4.1 (negative/positive; bad/good; unattractive attractive, $\alpha=.87)$.

\section{Results}

An ANOVA with language abstraction of the recommendation (DAV vs. IAV vs. SV vs. Adj) as between subjects variable on the buying intention revealed that participants' buying intentions for the ice cream increased with the level of abstraction used in the word of mouth communication (from concrete to abstract, respectively: $5.30,6.79,7.11,7.18, F(3,71)=3.02, p<.05)$. The attitude measure produced similar results: Receivers' attitudes toward the ice cream were more positive after receiving a more abstract statement (from concrete to abstract, respectively: 5.40, 5.96, 6.25, 6.67; $F(3,71)=3.75, p<.05)$.

\section{Discussion}

The findings of this study converge with those of Study 4.2, and show that positive word of mouth leads to higher purchase intentions and more favorable attitudes when it is worded in an abstract, rather than concrete, manner. The statements used in this study did not differ in terms of valence, which implies that the higher persuasive power of abstract (vs. concrete) word of mouth cannot be attributed to differences in valence.

\subsection{Mindset Moderates the Persuasiveness of Language Abstraction}

A question now is whether this means that a positive referral framed abstractly will always activate a more positive product attitude and a higher buying intention than a concrete version? In other words, should we all be talking abstractly to have the most impact? Probably not; word of mouth is an interactive and flexible process, and this has consequences for the language used. A particular message can be more or less 
persuasive depending on elements in the communication context, such as the objective a receiver's tries to accomplish with a product. Consumers can for example imagine using a product, such as drinking a cold beer on a hot summer's day, but they can also be focused on the outcome of product use, such as avoiding sunburn with sunscreen. Focusing on the thoughts and feelings about a desired outcome is known as outcome focus or outcome mindset (Escalas \& Luce, 2003, 2004; Pham \& Taylor, 1999; Taylor et al., 1998; Zhao, Hoeffler, \& Zauberman, 2007). In this mindset one is envisioning the end result that one wants to achieve (e.g., avoid sunburn). This focus is often used in advertising where consumers are encouraged to imagine the favorable outcomes from using a product (e.g., a recent Dutch lottery slogan reads: 'What would you do with 27 million Euro's?'). Conversely, a process focus/mindset refers to the construction of scenario's in which one considers all the specific steps which are necessary to attain a preferred objective.

Until recently, it was thought that a process focus stimulates superior goal attainment compared to outcome focused thought (Oettingen, 1995; Pham \& Taylor, 1999; Taylor et al., 1998). However, Escalas and Luce (2003) showed differential effects of mental simulation on behavioral intentions by varying involvement and argument strength. More specifically, under high involvement strong arguments had a greater effect on behavioral intentions than weak arguments when participants focused on the outcome of product use, while these effects were not found for process focus. In contrast, under low involvement they found an effect of strong over weak arguments for participants who were in a process (vs. outcome) focus.

This dissertation builds on previous research by examining under which conditions of message abstractness a process or outcome focus will lead to higher buying intention. My reasoning is based on research which suggested that people can more readily process information that is consistent (vs. inconsistent) with their expectations, such as stereotypes (e.g., Fiske \& Neuberg, 1990; Sherman, Lee, Bessenoff, \& Frost, 1998). I argue that a message is more persuasive when the language abstraction of the message is compatible with the mindset of the receiver. That is, process focused thought seems to encourage plan formation, creating a detailed stepby-step map of how actions $x$ and $y$ can lead to outcome $z$. Therefore, process focused thought will operate to increase sensitivity for information which is aimed at actions, information that precisely describes who does what in which way. Concrete language precisely describes information in an objective, non-interpretative fashion, with much indication about the specific observable situation and highly verifiable facts. Thus, 
when a receiver is considering the process of product usage, concrete product messages may be more effective than abstractly framed messages.

On the contrary, an outcome focus emphasizes the positive results of an action. In an outcome mindset the precise steps taken to get to the result are less important, and consumers are more focused on the end benefits. Abstractly framed product information describes the information as an endurable and broad conclusion, detached from the specific situation. Therefore, consumers in an outcome focus may be more sensitive to abstract language use. Conclusively, encountering information that is consistent with consumers' focus (e.g., they receive abstract information when focusing on the outcome, or they encounter concrete information while focusing on the process) will lead to a higher buying intention.

H6a In an outcome focus, an abstract product statement is more persuasive than a concrete message.

H6b In a process focus, a concrete product statement is more persuasive than an abstract message.

\section{Study 4.4: Effect of Receiver's Mindset on Effectiveness of Language Abstraction}

\section{Method}

\section{Design and Procedure}

One hundred forty students were randomly assigned to a 2 (mindset: outcome vs. process) x 2 (language abstraction: concrete vs. abstract) between subject design. The mindset of the participants was induced by asking them to imagine owning a house with a big lawn behind it, for which they decided that they needed an electric lawn mower to trim the grass. In the process mindset the participants were induced to think of using the product, while in the outcome mindset they were urged to think of the end product, a nicely mowed lawn (see Appendix $C$ for the outcome and process instructions). Both instructions contain the same amount of verbs and predicates to keep the language abstraction of two scenario's equal. Then the participants received a 
concrete or abstract statement about a Kamaro lawnmower. More precisely, the concrete and abstract descriptions were:

'A Kamaro lawnmower: mows your lawn easily, uses little electric power and lasts long.' (Concrete statement)

'A Kamaro lawnmower: is user friendly, reliable and environmentally friendly.' (Abstract statement)

\section{Dependent Variables}

After the product statement the participants were asked to indicate their attitude towards the Kamaro lawnmower on the three-item measure as in Study 4.1 and 4.3 (negative/positive; bad/good; unattractive attractive, $\mathrm{a}=.84$ ). Subsequently, participants indicated how likely they would buy this lawnmower, using the same 11point Juster scale as in Study 4.2 and 4.3. As manipulation check, participants indicated at the end of the test whether they thought the most about: (1) mowing the grass / (9) the end result after mowing the grass.

\section{Results}

\section{Manipulation checks}

As predicted, a T-test showed that participants in an outcome focus deliberated more about the end result of mowing the grass than participants in the process mindset $\left(M_{\text {outcome }}=7.26\right.$ vs. $\left.M_{\text {process }}=5.20, t(138)=5.97, p<.001\right)$.

\section{Product attitude}

An ANOVA with focus (outcome vs. process) and language abstraction (concrete vs. abstract) on product attitude showed the expected focus $x$ language abstraction effect $(\mathrm{F}(1,136)=7.64, \mathrm{p}<.01)$. Confirming Hypothesis 6a, planned contrast showed that participants who were in an outcome mindset rated the lawnmower as more positive when the advertised information was described abstractly (vs. concretely) $(F(1,136)=$ $4.26, p<.05)$, see Figure 16. As anticipated, the effects were in the opposite direction for the participants with a process focus (Hypothesis 6b): an abstract (vs. concrete) product statement activated a lower product attitude for participants in a process mindset $(F(1,136)=3.38, p=.06)$. 
$\square$ Concrete Description $\square$ Abstract Description

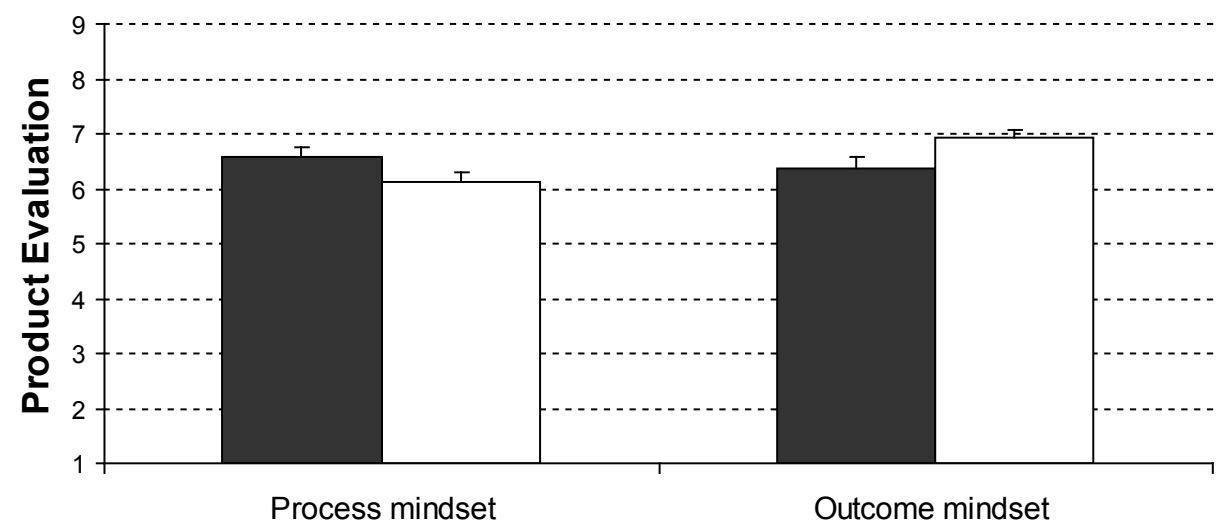

Figure 16.

Study 4.4. The interaction effect of mindset and language abstraction on product attitude.

\section{Buying intention}

Similar effects were found on the buying intention of the product: an ANOVA with mindset and language abstraction revealed a main effect of mindset on buying intention $\left(M_{\text {outcome }}=7.00\right.$ vs. $\left.M_{\text {process }}=6.34, F(1,136)=6.50, p<.05\right)$, and a mindset x language abstraction interaction effect $(F(1,136)=7.63, p<.01)$. As anticipated, planned contrast analyses revealed that an abstract (vs. concrete) product statement causes a higher buying intention for consumers who are in an outcome focus $(F(1,136)$ $=4.93, p<.05)$, see Figure 17. Additionally, the reversed pattern is marginally significant for the process focus: a concrete product statement leads to a higher buying intention than an abstract statement $(F(1,136)=2.80, p<.10)$. 
$\square$ Concrete Description $\square$ Abstract Description

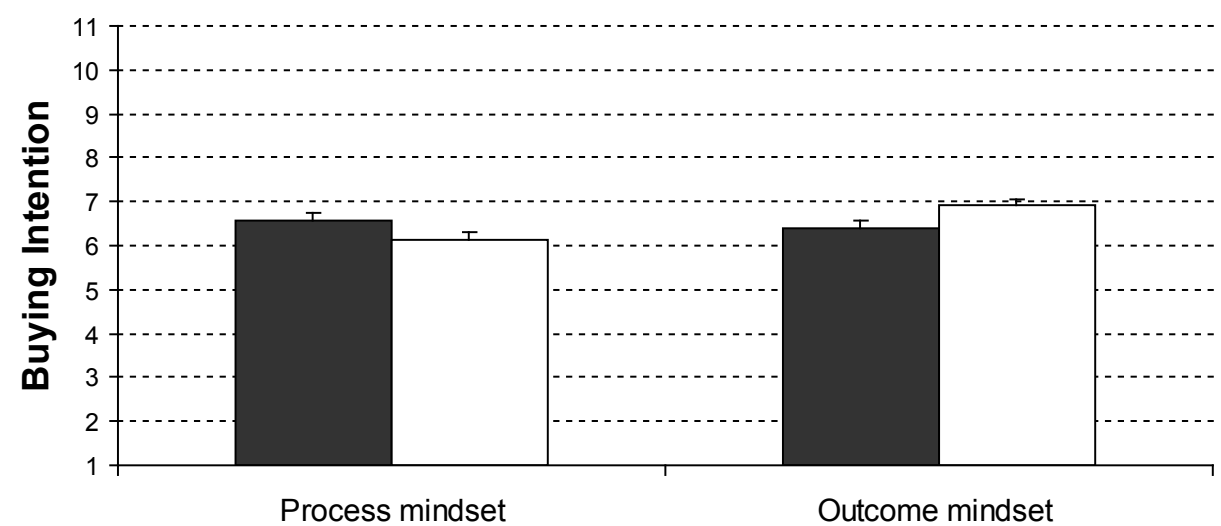

Figure 17.

Study 4.4. The interaction effect of mindset and language abstraction on product attitude.

\section{Discussion}

This study showed that the persuasiveness of language abstraction depends on the specific communication situation. The fit between language abstraction of a message and the focus of a receiver affects the impact of abstractness on receivers' product attitude and buying intention. When a consumer was focused at using the product (process focus) a concrete (vs. abstract) product message led to a higher buying intention and a more favorable product opinion. However, when consumers were thinking of the end benefits of a product (outcome focus), then an abstractly (vs. concretely) framed product message led to a higher buying intention and a more favorable product evaluation. In paragraph 4.5 and 5.2.3, I will elaborate on the results of this study and applicability of these findings in a marketing context, and discuss other moderators of the persuasiveness of language abstraction.

\subsection{General Discussion}

Previous research on language abstraction in social psychology mainly focused on the factors affecting the senders' use of language abstraction. The persuasiveness of language abstraction of product messages on receivers is additionally of main interest, especially for marketers. This chapter of the dissertation examined the effect of variations in language abstraction on the receiver's inference of the sender's product 
attitude, and the effect of more concrete or abstract language use on the receiver's product attitude and buying intention. It furthermore explored the moderating influence of the receiver's mindset on the effect on language abstraction on receiver's product evaluation, and excluded valence as a mediator for the effect of language abstraction on the receiver. These findings are exciting because they offer several important contributions to the current literature on the impact of language abstraction on receivers, and to research on word of mouth. Moreover, they also have clear implications for the formulation of persuasive messages.

Our first contribution is demonstrating that language abstraction in word of mouth influences the inferences that receivers make about the opinion that is held by the sender. More specifically, consumers inferred that senders have more favorable product attitudes when they heard a sender use more abstract language to describe a positive product experience. Conversely, if a sender used more abstract language to describe a negative product experience, receivers inferred that the sender held a less favorable attitude toward the product. In everyday life it is often important to understand other peoples' opinions. One tries to infer others' opinions in order to know for instance, whether one needs to be more convincing, whether others are lying or telling the truth, or whether the two of you are on the same page.

These findings provide communicators with some guidelines for adapting the language that they use when they share their product experiences with other consumers, but also with marketers. For example, if consumers want to make clear that they hold a really negative opinion about a brand or product (for example in a complaint letter or online review), they should communicate their negative experience in abstract terms. Along the same lines, reviewers wishing to present a balanced evaluation of a book or manuscript should pay attention to the level of abstraction with which they word positive and negative comments. In broader terms, the results of Study 4.1 indicate that receivers may use language abstraction as a cue for interpreting a sender's messages, while communicators could use language abstraction to enhance an audience's understanding of the message beyond its content.

Our second finding is perhaps even more relevant to the consumer behavior context. Studies 4.2 and 4.3 showed that buying intentions for a product to which consumers have no strong prior attitude were higher after receiving positive word of mouth that is worded more abstractly. For negative word of mouth, the opposite pattern was found, so that consumers' buying intentions for the discussed product were lower when the sender uses more abstract language. In other words, these 
studies demonstrate that the use of more abstract language increases the persuasiveness of word of mouth. These findings suggest that communicators who aim to maximize the positive impact of favorable word-of-mouth messages on buying intentions should use abstract language when formulating such messages. Conversely, communication about unfavorable aspects should be presented in concrete language, in order to minimize its negative impact on buying intentions. In paragraph 5.4 .2 of the general discussion, I will go into the practical relevance of these findings for marketing communication. As in Chapter 2, these findings cannot be attributed to the fact that abstract descriptions are more likely to have a stronger valence than concrete descriptions.

Our third main extension is showing how the focus of a receiver moderates for the second finding of this chapter on positive product referrals. The fit between language abstraction of a message and the focus of a receiver is shown to affect the impact of abstractness on receiver's product attitude and buying intention. More specifically, when a consumer was in a process focus, a product message framed concrete (vs. abstract) activated a higher buying intention and a more favorable product opinion, while in outcome focus an abstractly (vs. concretely) framed message stimulated a higher buying intention and product evaluation. In general, most products and services can be considered in light of both their use and in the end benefits of this use. For instance, one can imagine driving a car (process focus), and using it for the sake of getting from point A to point B (outcome focus). To maximize the impact of one's message, one should try to match the linguistic abstractness of one's communication to the receiver's needs. Marketers could activate a receiver's focus by the kind of information they provide, and by matching the language abstraction to the receiver's focus they can increase the persuasiveness of their communication. For instance, detailed information about specific attributes of a product direct the attention of the receiver on the process of product usage, and could therefore activate a process focus. When information like this is described concretely, the language abstraction of the message can maximize its impact on consumer behavior. In contrast, information aimed at higher order values or benefits can activate an outcome focus, and should therefore be framed abstractly (Means end chain: Reynolds \& Whitlark, 1995).

Other facets which may have considerable importance to the persuasiveness of language abstraction and are interesting for follow-up studies are the knowledge and attitude strength of a receiver. The impact of language abstraction may also 
depend on what a receiver is thinking, feeling, trying to achieve, and how much time and effort he wants to spend on processing the information. For instance, a strong opinion about a product could influence to what extend new information about this product is considered. One would expect that a receiver's attitude towards the product that is described in a message can have a considerable impact on the effectiveness of the language abstraction of the message. In situations in which the receiver agrees with the product message, an abstract framing which generalizes the information to the product or brand level may be more persuasive than a concrete message. In contrast, when a receiver disagrees with a product message, a concrete message may lead to less reactance and dispute, since it describes the information in a manner that is specific and situationally dependent, without a lot of subjective interpretation (I will elaborate on this matter in paragraph 5.2.3 and 5.4.2).

Conclusively, this chapter showed that the language abstraction of word of mouth can reveal information on the sender (i.e., strength of product attitude), is recognized by receivers and affects their behavioral intentions. Additionally, it was revealed that a receiver's mindset moderates the impact of linguistic abstractness. This confirms language abstraction serving as a subtle function in communication (Semin, 1994, 2000; Semin \& Fiedler, 1988, 1989, 1991). Future research should examine whether these findings can be generalized to advertising messages, but also to the use of abstract and concrete language in PR, or in communicating negative events (e.g., reputation threatening events in a company). 


\section{Chapter 5. General Discussion}

\subsection{Goal of the Dissertation}

The goal of this dissertation is to gain insight into word of mouth. To this end, language use during word of mouth was examined. I argue that above and beyond the valence of a message, the language used in a message may reveal a great deal about the sender of word of mouth, and have a significant effect on receivers. Abstractness is an essential aspect of language, and research on communication about interpersonal behavior revealed a systematic pattern in the use of language abstraction (e.g., Maass, et al., 1989, 1995; Douglas \& Sutton, 2003). The abstractness of language reveals information about the psychological and social processes underlying message production, and plays an important role in message perception (Semin \& Fiedler, 1988; Semin, 2000; Holtgrave \& Kashima, 2008). This dissertation demonstrates that language abstraction is a valuable and useful communication signal, and it's wellsuited to analyze product messages and improve our knowledge on word of mouth.

This dissertation examined when and why consumers use more abstract versus concrete language in word of mouth, and how these differences in language use affect the receivers of word-of-mouth messages. It showed that the language abstraction of word of mouth reveals information about the sender, such as the attitude of the sender about the object of communication. Moreover, consumers receiving word of mouth can pick up on subtle cues in the language abstractness of messages provided by the sending consumer and that they are affected by the language of word of mouth in their behavioral intentions.

\subsection{Scientific Implications and Future Research}

The findings of this dissertation offer several important contributions to the current literature on language abstraction, and the research on word of mouth. In general, the research adds to the growing stream of literature on linguistics in consumer behavior (e.g., Krishna \& Ahluwalia, 2008; Lowrey, 2007; Luna \& Peracchio, 2005; Puntoni, De Langhe, \& Van Osselaer, 2009), and the substantial body of research on message framing in marketing (e.g., Jain \& Posovac, 2001; Lee \& Aaker, 2004; Kim, Rao, \& Lee, 
2009; Maheswaran \& Meyers-Levy, 1990; Shiv, Britton, \& Payne, 2004). Additionally, the effects suggest that in research where the manipulation is communicated by means of language, such as in scenario and advertising studies, great care should be taken to keep the abstractness of the language similar across the manipulations. This way one avoids incorrect attribution of effects which are activated by language abstraction to other processes. This dissertation also opens new avenues for researchers who are interested in language abstraction and the role of language in consumer behavior.

Central topics in research on word of mouth have been the spread of word of mouth, and characteristics and motives of the source and receiver of word of mouth, such as source expertise, and the strength of the social ties between sender and receiver (Brown \& Reingen, 1987; Herr, et al., 1991). Studies focusing on the content and form of word of mouth are limited, and mainly examined the amount and valence of the product and service messages (Chevalier \& Mayzlin, 2006; East, Hammond \& Wright, 2007; Liu, 2006; Godes \& Mayzlin, 2004). This dissertation demonstrated that in addition to the valence of the message, the language of a message is an important and helpful communication signal. This adds to previous work on word of mouth by providing a framework to categorize word of mouth based on the language abstraction of the product messages. With this framework one can gain knowledge on the sender of word of mouth, and determine the impact of the product message on receivers.

\subsubsection{Effect of Senders' Product Attitudes on Language Abstraction in Word of Mouth}

The first empirical chapter of this dissertation examined consumer's use of language abstraction in word of mouth, and which mechanism drives this behavior (Research Question 1). I hypothesized that the level of abstraction that people use to describe a product experience is influenced by their a-priori attitude about the product in the experience. A series of six experiments with a total of approximately 700 participants showed that product experiences that are congruent with consumers' brand attitudes are communicated more abstractly than experiences that are incongruent with brand attitudes. More specifically, consumers used more abstract language to describe negative product experiences that involved a brand toward which they hold an unfavorable brand attitude compared to a favorable attitude. Positive product experiences were communicated more concretely by consumers with an unfavorable compared to favorable product attitude. 
A mixture of manipulations for the independent and dependent variables, and a wide range of product categories (ranging from durables to fast moving consumer goods) demonstrated the robustness of the systematic variation of language abstraction during word of mouth. For example, Studies 2.2 and 2.3 demonstrated this effect by manipulating brand attitudes with short descriptions, but participants' a priori opinions towards existing brands were used in Studies 2.1, 2.4 and 2.5. Moreover, the systematic variations in language abstraction were found when product experiences were generated by showing film clips of a student using a product (Studies 2.2, 2.3 and 2.4), but also when participants tested a product themselves (Study 2.1). Furthermore, this effect operates not only when consumers rated or chose from pre-defined statements (Studies 2.2 and 2.6), but also when they described product experiences in their own words (Study 2.5).

The approach of this dissertation diverges from previous research because it examines the systematic variation in language abstraction during object related communication. Where previous studies only focused on language abstraction in the descriptions of human behavior, I sought to establish that people also systematically vary language abstraction in communication about non animate objects, products in this case. Additional determinants of language abstraction in word of mouth which could be interesting for future research include the strength with which a product opinion is held, and the sender's expertise and involvement with the product category. When a novice describes a product experience, this person may use more concrete language. A novice only has little experience with the product, and concreteness describes the experience as situationally dependent. This way, the novice may avoid making claims about which he lacks the knowledge. Similarly, scoring high on product involvement and expertise should make it easier for consumers to generalize experiences to the product level, which should lead to an increase of the sender's use of abstract language.

A second important contribution of Chapter 2 is that it dealt with a criticism of previous research on language abstraction, which lies in the inherent relationship between the abstractness of a description and its valence. The valence of a product message could be argued as an alternative hypothesis for systematic variations in the use of language abstraction. Although the role of valence has been addressed in some previous work on language abstraction (Douglas \& Sutton, 2003, 2006), empirical evidence had not been conclusive. The valence-abstractness relationship seems to be an important issue to consider when studying language abstraction (e.g., when 
creating stimulus material). Many of the studies in Chapter 2 consistently show that the effect of product attitudes and experiences on language abstraction was not mediated by differences in the valence of the descriptions (e.g., Studies 2.1 and 2.5). More importantly, by experimentally manipulating the correlation between the abstraction and valence of descriptions, Study 2.2 conclusively demonstrates that the variation in language abstraction is not driven by differences in valence between concrete and abstract language.

The third goal of Chapter 2 was to explore the driving mechanism of systematic language use in word of mouth. Although this linguistic phenomenon itself is well established (Maass, et al., 1989, 1995, 1996; Sekaquaptewa et al., 2003; Webster, Kruglanski, \& Dwight, 1997), relatively little was known about the exact processes that underlies this systematic language use. Study 2.6 showed how systematic variations of language abstraction in word of mouth are driven by the fact that experiences which are in line with one's product attitude are more likely to be attributed to the product and not to the specific user or usage situation. This inference process mediated the interaction effect of expectation and product attitude on language abstraction, and I claim that this mechanism is the main driver of the systematic use of language abstraction.

\subsubsection{Language Variation in Sender-Receiver Dyad}

The language that consumers use in word of mouth need not only be driven by consumers' a priori product attitudes, but could also be affected by other elements in a sender-receiver dyad, as is shown in Chapter 3. This section of the dissertation showed the sender's adaptive use of language abstraction triggered by elements in a senderreceiver dyad (Research Question 2). Main elements in this dyad are the receiver (and his characteristics) to whom one communicates and the goal with which one communicates (Grice, 1975; Higgins, 1981; Schwarz, 1994). In extension to previous studies on strategic use of language abstraction, I focused on the effect of communication goals aimed at receivers on the use of language abstraction in word of mouth. In previous studies (Douglas \& Sutton, 2003; Wenneker, et al., 2005) the communication goal commonly focused the attention of participants at the levels of language abstraction. In the studies of this chapter, the communication served a more abstract goal, aimed at the product attitude of the receiver: the participants varied 
language abstraction to attend to the actual product attitude of the receiver, or to attain a desired attitude of the receiver.

Since the ability to tailor one's product messages is one of the reasons why word of mouth is such a powerful medium (Herr, Kardes \& Kim, 1991), consumers who engage in word of mouth should adapt their use of language abstraction to characteristics of their audience. Chapter 3 demonstrated that communicators use more abstract language when they describe an experience that is congruent (rather than incongruent) with the receiver's product attitude. More specifically, a positive product experience is described more concretely to a receiver with an unfavorable (vs. favorable) product attitude, while a negative experience is described more abstractly to a receiver with an unfavorable (vs. favorable) attitude. A phenomenon I labeled the Receiver Induced Systematic Language Abstraction. An interesting avenue for future research could be the strength of the relationship between sender and receiver. For instance, in a weak tie relationship one might avoid using very abstract product claims, since one may be unfamiliar with the product attitude of the receiver.

Chapter 3 also focused on the effects of consumers' communication goals on their use of language abstraction. I argued that consumers may modify their use of linguistic abstraction to guide the thoughts and feelings of the receiver and create a desired reality. Language can be used to direct the attention of the audience to a particular facet of reality: higher levels of abstraction emphasize the general qualities of the product. Accordingly, Study 3.2 found that consumers who have the goal of persuading others of the high quality of a product use more abstract language for positive product experiences, while negative information was described more concretely. Even though persuasion is an important goal in marketing and word of mouth, the effects of other communication goals on language abstraction could be considered in follow-up studies. For instance, a goal to self protect may be best served by concrete language, while a self enhancement goal may activate more abstractness.

In sum, the obtained linguistic patterns in Chapter 2 and 3 of this dissertation extended scientific literature on language abstraction by demonstrating the effects on the main elements in a word-of-mouth communication situation, such as the attitude and communication goals of the sender and the attitude of a receiver. It remains to be seen how these variables will interact. For instance, how abstractly will a consumer with a favorable product attitude communicate a positive product message to a consumer with an unfavorable product attitude? And would a consumer use more concrete or abstract language when one is trying to persuade a receiver with an 
unfavorable product attitude of a favorable view point? I argue that this depends on the particular communication context. Sometimes consumers will reason from their own perspective (Kruger, Epley, Parker, \& Ng, 2005; Ross \& Sicoly, 1979), and let their product attitude or communication objective control their choice of words. Then, the bias of language abstraction towards the attitude of the receiver might be weaker than the bias towards the attitude of the sender. At other times, the effect of the attitude of the receiver on the language use of the sender outweighs the effects of a sender's personal product opinion, for example, when a receiver has more power or the receiver's evaluation of the sender is of main importance.

\subsubsection{Effects of Language Abstraction on Receivers}

The results of Chapter 3 suggest that consumers may be implicitly aware of the effects of language abstraction on an audience. Chapter 4 examined the actual effects of language abstraction on consumers receiving word of mouth. This chapter probably forms the most important contribution of the current dissertation, because it is a large scientific extension on previous research on language abstraction and highly relevant for marketing. I started off from a two sided hypothesis since both abstract as well as concrete word of mouth could have been more persuasive. On the one hand, abstract language might be more influential, because an abstract description frames product information as a stable quality of the product, dispositional rather than situational dependent (Semin \& Fiedler, 1988). On the other hand, abstract descriptions are more open to dispute and less verifiable, while concrete descriptions are often more vivid, and vividness has been shown to have a persuasive effect (Kim, Kardes, \& Herr, 1991; Kisielius \& Sternthal, 1984). Chapter 4 revealed in which communication situation concrete or abstract language is more convincing, and holds three mayor findings.

First, extending previous empirical work (e.g., Douglas \& Sutton, 2006, 2010;

Reitsma-Van Rooijen, Semin, \& Van Leeuwen, 2006) receivers have been shown to distill information about a sender from the language abstraction of a product message. It is important to stress that inferring a sender's product attitude from a word-ofmouth message is a complicated task in which several communication motives could affect the content and the form of a product message (Sundaram, et al., 1998). Receivers are aware of these possible factors to which they can attribute the use of concrete or abstract language in word of mouth. However, in spite of this, Study 4.1 found that abstract negative word of mouth led receivers to infer that the sender had a 
less favorable product attitude, and from abstract positive word of mouth receivers inferred that the sender of word of mouth had a more favorable product attitude. This means that abstract language seems to suggest more strong or outspoken opinions.

The second contribution of this chapter is an enhanced understanding of the effects of language abstraction on the receivers' behavioral intentions. To my understanding, this dissertation provides the first evidence of an effect of language abstraction on receivers' opinions about the described object and behavioral intention towards it. In previous research on language abstraction, there has been only limited attention for the effects of language abstraction on receivers. Studies 4.2 and 4.3 revealed how language abstraction moderates the effect of word of mouth on receivers. A positive recommendation framed abstractly is shown to cause a higher purchase intention than the same message framed concretely, while negative word of mouth described concretely incited a higher purchase intention than an abstract version. These findings are exciting, because they introduce language abstraction as a moderator of the persuasive impact of word of mouth (and answer Research Question 3). The effects have also been shown to remain significant after correcting for the influence of differences in valence between concrete and abstract descriptions. This thus excluded valence as a mediator for the effect of language abstraction on the receiver. Additionally, these results have clear implications for the formulation of persuasive messages, which will be described in paragraph 5.4.

The third main contribution is demonstrating how mindset moderates the persuasiveness of language abstraction. More specifically, when a receiver was in a process focus (i.e., thinks of using a product) a concretely framed product message was shown to be more persuasive compared to an abstractly framed message. Conversely, when a receiver was in an outcome focus (i.e., contemplates the end results one will gain from using a product) an abstract message had a stronger impact on receiver's product attitude and buying intention than a concrete version. This means that abstract positive word of mouth is not always more persuasive, but the impact of the abstractness of language depends on specific elements in the communication situation, such as the receiver's mindset.

How can this understanding of language abstraction and consumers mindset be used to maximize the impact of one's message? Overall, consumers can look at and think about products and services in both a process and an outcome mindset. Some products and services, however, may automatically provoke a process or outcome mindset. For instance, insurances are more likely to trigger an outcome mindset: 
'preventing having to pay a large sum of money when something goes wrong'. Only the end goal is essential, the process is less interesting. In these cases, one is well advised to fit the language abstractness of a message to the mental focus that their product activates (e.g., abstract language in case of the insurance company). However, most products can probably be considered in both mindsets. People can consider taking vitamin pills (e.g., which ones, when, how) or consider the improvement in one's health as an end result of taking vitamin pills. Therefore, the specific communication situation is of main importance; one should match the abstractness with the receivers needs. A company's marketing communication can also activate a process or outcome focus. For instance, messages communicating higher end values and benefits are more likely to activate an outcome focus, so that an abstract product description will maximize the persuasiveness of such messages.

There are also other moderators in the persuasiveness of language abstraction that could be considered interesting, such as the strength of the product attitude of a receiver and the receiver's cognitive capacity. This dissertation focused on receivers who have no prior opinion about the products under discussion (i.e., new brand users). However, consumers frequently have an opinion about a product (e.g., other brand loyals; Rossiter \& Bellman, 2005), which may be based on advertising, direct experience, or previous word of mouth. An existing opinion about a product could influence to what extend new information about this product is considered. When word of mouth is in line with the receiver's product attitude, then the effects of language abstraction may be enhanced. For example, a more abstract version of a positive message stresses the generalizability of the information that a receiver with a positive product opinion agrees with. In this case, an abstract message is probably more persuasive than a concrete version.

The predictions for the impact of linguistic abstractness of a product message which is inconsistent with a receiver's product opinion are less clear-cut. On the one hand, one could argue that people avoid, ignore or pay less attention to information that is incompatible with their existing attitude than to information that matches their attitudes (Selective Exposure Hypothesis: Frey, 1986; Festinger, 1964). Additionally, preference-inconsistent information is seen as less valid (Ditto et al., 1998; Ditto \& Lopez, 1992), and is counter argued more (Jain \& Maheswaran, 2000). Consequently, consumers may not be open to receiving word of mouth which counters their current product attitude (Ahluwalia, 2000), and subtle differences in the framing of preference-inconsistent information, such as the language abstraction, may therefore 
have little effect. On the other hand, one could argue that a concrete description is an objective and verifiable way to share information free from any subjective interpretation. This makes a concrete description less likely to be counter argued by receivers than an abstract description. Thus, when information counters a receiver's attitude, then a concrete message may be accepted more easily, and activate less disagreement than an abstract message. Accordingly, one could conclude that preference inconsistent information ought to be presented concretely. Further studies should reveal the effect of a receiver's product attitude on the persuasiveness of language abstraction.

Future research should also examine whether the findings of this dissertation in consumer-to-consumer communication can be generalized to advertising messages and PR in which a marketer communicates to a consumer. I assume that my findings will hold in many business-to-consumer communication situations, but they probably cannot straightforwardly be generalized to all business-to-consumer communication (advertising). A main element in the effectiveness of word of mouth is the trustworthiness of the source, and I propose that this variable plays a central role in my work. When the trustworthiness of the source is dubious, as in a persuasion situation with a sales person who may have an ulterior persuasion motive, the abstractness of a message may interact with consumer's persuasion knowledge. Persuasion knowledge refers to consumers' assumptions and beliefs about persuasion and marketers' motives, strategies, and tactics (Campbell, 1999; Campbell \& Kirmani, 2000, 2008; Friestad \& Wright, 1994). This includes the effectiveness and appropriateness of marketer's persuasion tactics, and coping strategies for these persuasion attempts. Basically, consumers use persuasion knowledge to identify if someone is attempting to influence them.

How may the impact of language abstraction depend on persuasion knowledge? I propose that when persuasion knowledge is activated through an environmental cue, such as a source of a message having an ulterior persuasion motive, then abstract language will not lead to a more favorable attitude than concrete language. For instance, when a sales person communicates a more abstract referral about a product to a customer, than the implications of the abstractness can cause the customer to infer that the sales person is trying to influence them. Overall, an abstract referral frames the information as a general and stable quality of the product (Semin \& Fiedler, 1988). So, when positive brand information is described abstractly, it suggests that the overall brand is positive. The customer might judge the sales person as more 
manipulative and deceitful when this person communicates an interpretation of the situation. A more concrete description, on the other hand, is a more situational description which is highly verifiable, and therefore a customer might not see the sales person as dishonest. Thus, I argue that persuasion knowledge may moderate the effect of language abstraction on consumer's product attitude. More specifically, when environmental cues indicate a persuasion motive then a positive product referral framed abstractly may incite a lower product attitude than a concrete description. Future research should explore how language abstraction interacts with source trustworthiness, and what the effect of language abstraction is on persuasion knowledge and consumer behavior.

One may argue that there are several similarities between language abstraction and construal level, and therefore I would like to take a page to draw attention to the similarities and distinction between both constructs. Construal level theory suggests that people use concrete low-level construals for near events, and abstract high level construals for distant events (Liberman \& Trope, 1998; Trope, Liberman, \& Wakslak, 2007). Low-level construals are contextualized representations, rich in detail and relatively unstructured, linked to the subordinate purpose of the 'how' of the activity (i.e., writing down things), whereas high-level construals are decontextualized and schematic, and linked to the super ordinate means, the 'why' of the activity (i.e., getting organized; Vallacher \& Wegner, 1989). Several researchers examined construal levels in marketing research (Hamilton \& Thompson, 2007; Lee, Keller \& Sternthal, 2010; Zhao, Hoeffler \& Zauberman, 2007), and showed for instance that abstract 'why'-laden messages are highly persuasive when a voter's decision is temporally distant, while concrete 'how' appeals are more persuasive with an imminent decision (Kim, Rao, \& Lee, 2007).

Construal level theory and language abstraction of the linguistic category model can be matched. Using more abstract language can be a method to communicate a high level construal, since abstract language stresses the stability and generalizability of the information. Conversely, concrete language can be used to share a low-level construal, which specifically describes the detailed steps of an event. However, low and high construal can also be described in the same level of language abstraction. For example, the construal level examples (low: 'writing down things' vs. high: 'getting organized') would both be rated as concrete messages according to the linguistic category model, because 'writing' and 'getting' are both Action Verbs. Hence, where research on construal level focused on more concrete and abstract 
actions and examined concrete- and abstractness in its broad meaning, language abstraction according to the linguistic category model focuses on the specific way a message is communicated (i.e., with which words). Language abstraction evolves around speaking more concrete or abstractly, not solely about meaning something more concrete or abstractly. Future research could examine if the effects found with construal level can also be established with language abstraction, such as the relationship with regulatory focus (Lee, Keller, \& Sternthal, 2010) and temporal distance (Kim, Rao, \& Lee, 2008).

Conclusively, this dissertation is the first to apply the linguistic category model outside the context of (inter)personal behavior, and demonstrated the impact of language abstraction on consumers. Chapter 2 demonstrated systematic variations in the sender's use of language abstraction in word of mouth, it showed the underlying mechanism and excluded an important alternative explanation for the phenomenon. Chapter 3 examined the impact of the sender-receivers dyad, particularly the impact of the product attitude of the receiver and the desired product attitude of the receiver on the senders' use of language abstraction. I completed the communication cycle from sender to receiver in Chapter 4, which demonstrated how language abstractness affects receivers of a word-of-mouth message.

\subsection{Research Limitations}

A first limitation of this dissertation pertains to the number of levels of the linguistic category model. Most of the studies of this dissertation used the four levels of the linguistic category model of Semin and Fiedler (1988). Since the introduction of the linguistic category model, both Semin (1994) and Carnaghi and colleagues (2008) suggested to add an extra level to the four level model. Semin (1994) initiated the 'State Action Verb', a level in between the IAV (level 2) and the SV (level 3), which is an unspecified action by the sentence subject which leads to state in the sentence object (e.g., 'you amaze me'). However, these types of verbs do not differ significantly in abstraction level from IAVs (Semin \& Fiedler, 1991), and according to Semin's manual, this extra level is very rare in the Dutch language. So, there was no strong need for us to study this level. The extra level of Carnaghi, et al. (2008) was a fifth level, labeled 'Nouns', above the highest level of the standard model (Adj). In the manual of the traditional four level model, nouns were included in the Adjectives level. At the highest level of language abstraction in the studies of this dissertation adjectives are 
mainly varied between the positive and negative experiences. In concurrence with Carnaghi's findings, an addition of a fifth level of nouns to my studies would probably enhance the power of the effects. In nearly all publications on language abstraction the four levels of the traditional linguistic category model are used. Since there were no theoretical reasons to deviate from the four level model, I decided to stick to the standard four level model. I also do not predict theoretical differences in my findings from the inclusion of another level of language abstraction.

A second limitation is related to the method applied. All of the studies of this dissertation are lab experiments. This allowed me to gain insight in the processes underlying the use of language abstraction, and the effects of variations in language abstraction on receivers. Uncovering this knowledge would have been nearly impossible with other research methods. To demonstrate the robustness and to expand the external validity of my findings, several manipulations for the independent variables and different dependent variables were employed. For instance, next to measuring the effects on language use by pre-determined descriptions (e.g., Study 2.1 and 2.2), the language abstraction of open responses was coded in Study 2.5. Additionally, there were several measures to assess the effects of language abstraction on receivers, such as the inferred product attitude of the sender, and product evaluation and buying intention of the receiver (e.g., Study 4.2 and 4.3). Notwithstanding, field data could improve the ecological validity of my findings. An interesting domain for studying language abstraction may be online word-of-mouth (a.k.a. word of mouse), such as product-related blogs or customer reviews. The linguistic pattern that was established in this dissertation is expected to also appear in natural settings. Research on language abstraction in online word of mouth should extend existing work, which has focused mainly on valence and frequency of conversations (e.g., Godes \& Mayzlin, 2004; Liu, 2006). For instance, one could analyze the language abstraction of positive and negative online reviews and compare the language use to star ratings. One would expect the highest amount of stars to correspond to the positive reviews framed abstractly, and the lowest amount to negative reviews framed abstractly, while concrete reviews would fall in between these two.

A related limitation is the lack of actual word-of-mouth conversations in the studies of this dissertation. In my research all the elements of a word-of-mouth referral are studied in separate experiments. For instance, participants tested a product in Study 2.1, they described a product experience in their own words in Study 2.5, and 
heard a receiver's voice uttering his/her product opinion on an audio recording in Study 3.1. Nevertheless, situations in which two consumers were actually talking to each other were not examined. I speculate, however, that in the abundance of evidence provided for systematic language use in word of mouth, studying an actual conversation would yield similar results.

\subsection{Practical Implications}

How can this thesis help marketers in dealing with word of mouth? And what does this thesis on language abstraction in word of mouth suggest for language use in marketing communication?

Beyond the theoretical significance of language use in word of mouth, the findings of this dissertation have important practical implications. Since word of mouth has a strong effect on sales, it is interesting to know what the possibilities are to affect these consumer conversations. I offer language abstraction as a tool to understand and influence the word of mouth about one's product, and suggest how marketers should employ language abstraction in their communication activities. I will describe a company's role from a more passive observer to an active mediator of word of mouth (Godes et al., 2005), and suggest various implications for marketing communication.

\subsubsection{Practical Implications for Word of Mouth Marketing}

To passively learn from word of mouth, marketers could analyze the language abstraction that consumers use in product messages, such as reviews and blogs, to help assess the underlying attitude of the author. Variations in language abstraction among senders could be used to determine the extent to which a product experience was congruent of incongruent with a consumer's expectations. For example, if an unfavorable review is written in concrete (rather than abstract) language, one could infer that the writer is generally positive about the brand or product. Thus, when customer service agents or webcare teams encounter a complaint about the company's service concretely, this person is well advised to pull out all the stops to make this happy customer again, because there is a high chance of success. However, complaints phrased in abstract terms could be an indication of an a priori negative service opinion. This means that it may be harder to compensate or please this customer, and 
one could expect less chance of success. This knowledge can be used to allocate time and energy of customer service agents.

Next to a natural tendency to vary language abstraction for product information based on one's product attitude, consumers can also choose to strategically use language abstraction to communicate product experiences. If a consumer wants to express a moderate positive product evaluation, one better use concrete language for positive product information. While an extremely positive product attitude is more likely to be picked up by receivers when one communicates this favorable information abstractly. In broader terms, communicators could use language abstraction to enhance an audience's understanding of the message beyond its content. This knowledge about language abstraction could also be interesting, for example, to politicians.

For a more active role in managing the word of mouth about one's product, this research also provides some suggestions. The first question would be: 'how do I want consumers to talk about my product?' This dissertation claims that companies should most of the times prefer consumers to use abstract language when formulating favorable product messages, and concrete language when communicating unfavorable product information. However, the preferred level of language abstraction also depends on the specific communication situation. Additional information about receivers can be used to employ language abstraction in one's advantage, a matter which will be more elaborately discussed in paragraph 5.4.2 practical implications of marketing communication.

The related second question is 'how do I get consumers to talk about my product with the desired language abstractness?' This dissertation has shown that a persuasion goal can affect the senders' use of language abstraction. Marketers could also activate a certain communication objective in commercials or direct marketing, which can result in the desired word of mouth about one's product. For example, one could reward one's customers recommendation to others (e.g., with gifts or discounts; Biyalogorsky, Gerstner, \& Libai, 2001; Rye \& Feick, 2007), to urge one's customers with a positive brand attitude share their positive experiences with others, which they will then do framed abstractly. In doing so, you are boosting the natural tendency of language abstraction during word of mouth.

Another possible technique to attain the preferred level of abstraction in word of mouth is to induce or prime the proper level of abstractness. Regulatory focus (Lee, Keller, \& Sternthal, 2010), affective feelings (Beukeboom \& Semin, 2006) and 
proprioceptive cues (i.e., bodily feelings; Beukeboom \& De Jong, 2008) have been shown to give rise to language abstractness. Moreover, exposure to concrete terms could lead to the use of more concrete language, while more abstract language can trigger the use of more abstractness (Stapel \& Semin, 2007). Thus, marketers can try to facilitate consumers to spread word of mouth about their brand at the desired level of language abstraction.

\subsubsection{Practical Implications for Marketing Communication}

This dissertation focused on language abstraction in consumer-to-consumer communication. An interesting possibility is to expand these findings to a market level. This section holds several recommendations on the use of language abstraction in marketing communication. I propose that the findings of this dissertation will hold in many business-to-consumer communication situations. Previously, it was explained that effectiveness of language abstraction partly depends on the activation of persuasion knowledge. Not all business-to-consumer communication situations activate persuasion knowledge (Campbell \& Kirmani, 2000). For instance, persuasion knowledge will not be activated when ulterior motives are less accessible or when consumers are cognitively constrained. The basic findings of this thesis suggest that in order to paint a positive picture of a brand or product, companies have most to gain from framing favorable product messages in PR and advertisements abstractly, and presenting unfavorable product information concretely. For instance, Toyota is well advised to communicate product recall and malfunctioning car parts in a concrete form.

Furthermore, when deliberating the language abstraction of marketing communication, marketers should take the specific target audience to whom they are communicating into consideration. A broad distinction in customer target groups is brand loyals, other brand loyals/switchers and new category users (Rossiter \& Bellman, 2005). Brand loyals represent a company's core of sales, the most frequent buyers of their products, and other brand loyal/switchers are the people who buy products of the company's competitors. How should a company employ language abstraction in communication to their brand loyals? And what are the differences in linguistic abstractness of messages to one's brand loyals and other brand loyals? Or what to say in one's communication to new brand users compared to other brand loyals? Since this dissertation examined the effects of language abstraction on receivers 
who have no prior product opinion, it can be assumed that in marketing communication to new brand users the findings will hold. That is, to attain a positive product evaluation positive messages about one's brand or company should be framed abstractly and negative messages framed concretely. When one can distinguish the objective of a new brand user, then one could even adjust the abstractness of one's positive message to this (i.e., concrete messages for receivers in a process focus and an abstract message to the ones in an outcome focus). This can also be linked to advertising focused on product usage or on end goals. More specifically, advertising which is focused at using a product is best served with concrete language, while advertising focused at the outcome of using a product is probably most persuasive when framed abstractly.

Moreover, in line with my reasoning in paragraph 5.2.3, I propose that the findings of this dissertation will also hold for communication to one's brand loyals. Thus, messages which are compatible with a receiver's product attitude should be described abstractly, and incompatible messages concretely. Even more so, I expect the persuasiveness of language abstraction to be stronger for brand loyals than new category users, since the abstractness stresses the congruence between the product information and their product attitude (cf. Rubini \& Sigall, 2002).

In communication to other brand loyals and other brand switchers I advise caution in the use of language abstractness. As previously explained (paragraph 5.4), the effectiveness of language abstraction of information that is incompatible with receivers product opinion remains uncertain. For instance, when a company communicates positive information about their brand to an other brand loyal, will a concrete message activate a more positive brand opinion than an abstractly framed message? Or will there be no effect of language abstraction in this particular situation? I recommend describing positive information about one's company or brand to consumers with a negative product attitude concretely, because concrete information will activate less resistance and dispute. Unfavorable company information should also be described concretely, to suggest that the information is situational dependent and should not be seen as a stable characteristic of the product.

Conclusively, this means that negative company, brand and product information should always be described concretely, independent of the target audience. With positive information it depends on the particular communication context: in general positive product messages should be framed abstractly to new brand users and brand loyals, and concretely to other brand loyals and other brand 
switchers. However, when product use is central for a new brand user (process focus), then a concretely framed message could be more persuasive than an abstract version.

\subsection{Conclusion}

Word of mouth, an intriguing phenomenon. The rewards of good word-of-mouth marketing can be gigantic and excel those of other marketing approaches. This fascination has led to a lot of research, and I have yet to meet a marketing researcher who did not consider word of mouth interesting. The phenomenon is however also undeniably complex and challenging to study. A starting point to understand and manage word of mouth is to comprehend what is being communicated about products and services, and how this is said. I offered a framework to classify the language of word of mouth, which informs us what consumers' product attitudes may be, how they are likely to pass on messages, and what impact those messages may have. It is one way to gain insight into consumer minds and to determine the impact of word of mouth on receivers. This knowledge can help to improve companies' service and to manage word-of-mouth marketing. Additionally, I also hope that my linguistic approach will inspire others to consider language use in future research on word of mouth and in consumer behavior in general. 



\section{Appendix}

\section{Appendix A. Statements used in Chapter 2}

The product descriptions according to the four levels of the linguistic category model are described in increasing order (from concrete to abstract: descriptive action verb, interpretive action verb, state verb, and adjective). The descriptions of the negative experiences are presented within the brackets.

\section{Statements used in Study 2.3}

Communication network

Sofie calls [cannot call] on the IDEO network

Sofie communicates [cannot communicate] on the IEDO network

Sofie is [dis]pleased with the IDEO network

The IDEO network has good [poor] coverage

T-shirt

The Evic T-shirt is pink [turned pale]

The Evic T-shirt remained the same [changed color]

The Evic T-shirt pleases [does not please] Sofie

The Evic T-shirt is of good [low] quality

MP3 player

The Trevor plays an MP3 file

The Trevor MP3 player works [not] well

The Trevor MP3 player [dis]pleases Sofie

The Trevor MP3 player is [not] a good product

Yoghurt

Sofie tastes [did not taste] the Fresco yogurt

Sofie consumes [did not consume] the Fresco yogurt

Sofie likes [did not like] the Fresco yogurt

Fresco yogurt is [not] tasty 
Appendix

Statements used in Study 2.6

Dish washer

Sofie takes the glasses out of the Tripal dish washer

Sofie discovers that the glasses have become clean [remained dirty] in the Tripal dish washer

Sofie is [dis]pleased with the Tripal dish washer

The Tripal is a good [bad] dish washer 


\section{Appendix B. Statements used in Chapter 3}

The product descriptions according to the four levels of the linguistic category model are described in increasing order (from concrete to abstract: descriptive action verb, interpretive action verb, state verb, and adjective). The descriptions of the negative experiences are presented within the brackets.

\section{Statements used in Study 3.1}

Communication network

Sofie calls [cannot call] on the IDEO network

Sofie communicates [cannot communicate] on the IEDO network

Sofie is [dis]pleased with the IDEO network

The IDEO network has good [poor] coverage

Yoghurt

Sofie tastes [did not taste] the Fresco yogurt

Sofie consumes [did not consume] the Fresco yogurt

Sofie likes [did not like] the Fresco yogurt

Fresco yogurt is [not] tasty

\section{Statements used in Study 3.2}

Communication network

Sofie calls [cannot call] on the IDEO network

Sofie communicates [cannot communicate] on the IEDO network

Sofie is [dis]pleased with the IDEO network

The IDEO network has good [poor] coverage

Dish washer

Sofie takes the glasses out of the Tripal dish washer

Sofie discovers that the glasses have become clean [remained dirty] in the Tripal dish washer

Sofie is [dis]pleased with the Tripal dish washer

The Tripal is a good [bad] dish washer 


\section{Appendix C. Statements used in Chapter 4}

The product descriptions according to the four levels of the linguistic category model are described in increasing order (from concrete to abstract: descriptive action verb, interpretive action verb, state verb, and adjective). The descriptions of the negative experiences are presented within the brackets.

\section{Statements used in Study 4.1}

Perceived language abstraction of positive statements (pretest) are presented within the brackets at the end of each statement.

\section{Razor blade}

I shave with Shaft razor blades [the Shaft razor blades do not glide well] (2.44)

I use Shaft razor blades [the Shaft razor blades do not function well] (2.80)

I [do not] like Shaft razor blades (5.07)

Shaft [does not have] has nice razorblades (5.74)

Dessert

I [merely] tasted the Moccalicious dessert (2.75)

I finished [did not finish] the Moccalicious dessert (3.34)

I liked [did not like] the Moccalicious dessert (4.64)

The Moccalicious dessert is [not] tasty (5.18)

\section{Computer}

My Japca computer runs [does not run] my software programs [well] (2.19)

My Japca computer functions [does not function] well (3.51)

My Japca computer pleases [does not please] me (5.05)

My Japca computer is of high [mediocre] quality (5.13)

Car

A Katasca car does [not] rust (2.23)

A Katasca car lasts [does not last] long (3.65)

I consider a Katasca car to be [un]reliable (4.75)

A Katasca car is a high [low] quality car (5.60) 


\section{Statements used in Study 4.2}

Perceived language abstraction of positive statements (pretest) are presented within the brackets at the end of each statement.

\section{Mortgage broker}

'An acquaintance is about to acquire a property and consulted the mortgage broker Snyder. He shares his experience with this broker with you.'

Mortgage broker Snyder told [did not tell] me which mortgage is the best (2.75)

Mortgage broker Snyder helped [did not help] me with finding the best mortgage

I am [not] satisfied with mortgage broker Snyder (5.05)

Snyder is a reliable [an unreliable] mortgage broker (5.55)

\section{TV program}

'Somebody shares his opinion about the TV series 'PS' with you.'

I watched [did not watch] the TV soap 'PS' (2.48)

I have been following [did not follow] the TV soap 'PS' (3.20)

I thought the TV soap 'PS' was [not] exciting (4.57)

'PS' was [not] an exciting TV soap (6.02)

Club

'A classmate went to club Jack in the city centre of [X] last weekend. She shares her experience with you.'

I danced [did not dance] in club Jack (1.77)

I stayed [did not stay] in club Jack (2.91)

I had [did not have] a good time in club Jack (4.82)

Jack is [not] a nice club (5.84)

\section{Detergent}

'You are in the supermarket for detergent and run into an acquaintance who shares the following about Twist detergent.'

I wash [do not wash] my dishes with Twist detergent [anymore] (2.25)

I [do not] use Twist detergent (3.20)

I am [not] satisfied with Twist detergent (4.82)

Twist is [not] a fine detergent (5.91) 


\section{Instructions for Outcome and Process Mindset used in Study 4.4}

\section{Outcome mindset}

'Imagine the end result after mowing the grass: the advantages of a trimmed lawn and how it feels when your lawn looks perfect after mowing it. Thus, think about the end result and how it feels after mowing the grass.'

\section{Process mindset}

'Imagine yourself mowing the lawn: walking on the grass, smelling the freshly mowed grass, and plan how you would cut it. Imagine doing this every two weeks at summertime. Thus, think about using the lawnmower and how it feels to use this mower.' 


\section{References}

Aggarwal, P., \& Law, S. (2005). Role of relationship norms in processing brand information. Journal of Consumer Research, 32, 453-64.

Ahluwalia, R. (2000). Examination of psychological processes underlying resistance to persuasion. Journal of Consumer Research, 27(2), 217-232.

Anderson, E. W. (1998). Customer satisfaction and word of mouth. Journal of Service Research, 1(1), 5-17.

Arndt, J. (1967). Word of mouth advertising and informal communication. In Cox, D.F. Risk taking $\mathcal{E}$ Information handling in consumer behaviour; Boston University, 188-239.

Bansal, H. S. \& Voyer, P. A. (2000). Word-of-mouth processes within a services purchase decision context. Journal of Service Research, 3(2), 166-177.

Beukeboom, C., \& de Jong, E. (2008). When feelings speak - How affective and proprioceptive cues change language abstraction. Journal of Language and Social Psychology, 27(2), 110-122.

Beukeboom, C., \& Semin, G. (2006). How mood turns on language. Journal of Experimental Social Psychology, 42, 553-566.

Biyalogorsky, E., Gerstner, E., \& Libai, B. (2001). Customer referral management: Optimal reward programs. Marketing Science, 20(1), 82-95.

Blankenship, K., \& Holtgraves, T. (2005). The role of different markers of linguistic powerlessness in persuasion. Journal of Language and Social Psychology, 24(1), 324.

Bone, P. (1995). Word-of-mouth effects on short-term and long-term product judgments. Journal of Business Research, 32(3), 213-223.

Brennan, S. E., \& Williams, M. (1995). The feeling of another's knowing: prosody and filled pauses as cues to listeners about the metacognitive states of speakers. Journal of Memory and Language, 34(3), 383-398. 
Brown, J., Broderick, A., \& Lee, N. (2007). Word of mouth communication within online communities: Conceptualizing the online social network. Journal of Interactive Marketing, 21(3), 2-20.

Brown, T., Barry, T., Dacin, P., \& Gunst, R. (2005). Spreading the word: Investing antecedents of consumers' positive word-of-mouth intentions and behaviors in a retailing context. Journal of Academy of Marketing Science, 33(2), 123-138.

Brown, J., \& Reingen, P. (1987). Social ties and word-of-mouth referral behavior. Journal of Consumer Research, 14 (3), 350-362.

Campbell, M. (1999). Perceptions of price unfairness: Antecedents and consequences. Journal of Marketing Research, 36(2), 187-199.

Campbell, M., \& Kirmani, A. (2000). Consumers' use of persuasion knowledge: The effects of accessibility and cognitive capacity on perceptions of an influence agent. Journal of Consumer Research, 27(1), 69-83.

Campbell, M. \& Kirmani, A. (2008). I know what you're doing and why you're doing it: the use of the persuasion knowledge model in consumer research," in Haugtvedt, Curt, Herr, Paul and Frank Kardes (eds.), Handbook of Consumer Psychology, Psychology Press: New York, 549-574.

Carnaghi, A, et al. (2008). Nomina sunt omina: On the inductive potential of nouns and adjectives in person Perception. Journal of Personality and Social Psychology, 94(5), 839-859.

Chandon, E., \& Janiszewski, C. (2009). The influence of causal conditional reasoning on the acceptance of product claims. Journal of Consumer Research, 35 (May), 10031011.

Chevalier, J., \& Mayzlin, D. (2006). The effect of word of mouth on sales: Online book reviews. Journal of Marketing Research, 43, 345-54.

Corfman, K. (1991). Comparability and comparison levels used in choices among consumer products. Journal of Marketing Research, 28(3), 368-374.

De Bruyn, A., \& Lilien, G. L. (2008). A multi-stage model of word-of-mouth influence through viral marketing. International Journal of Research in Marketing, 25(3), 151-163.

Matos, C. A, \& Rossi, C. (2008). Word-of-mouth communications in marketing: a metaanalytic review of the antecedents and moderators. Journal of the Academy of Marketing Science, 36(4), 578-596. 
Dellarocas, C. (2003). The digitization of word of mouth: Promise and challenges of online feedback mechanisms. Management Science, 49(10), 1407-1424.

Dichter, E. (1966). How word-of-mouth advertising works. Harvard Business Review, nov-dec, 147-166.

Ditto, P., \& Lopez, D. (1992). Motivated skepticism - Use of differential decision criteria for preferred and nonpreferred conclusions. Journal of Personality and Social Psychology, 63(4), 568-584.

Ditto, P. H., Scepansky, J. A., Munro, G. D., Apanovich, A. M., \& Lockhart, L. K. (1998). Motivated sensitivity to preference-inconsistent information. Journal of Personality and Social Psychology, 75, 53-69.

Douglas, K., \& McGarty, C. (2001). Identifiability and self-presentation: Computermediated, communication and intergroup interaction. British Journal of Social Psychology, 40, 399-416.

Douglas, K., \& McGarty, C. (2002). Internet identifiability and beyond: A model of the effects of identifiability on communicative behavior. Group Dynamics: Theory, Research, and Practice, 6(1), 17-26.

Douglas, K. M. \& Sutton, R. M. (2003). Effects of communication goals and expectancies on language abstraction. Journal of Personality and Social Psychology, 84 (4), 682-96.

Douglas, K. M. \& Sutton, R. M. (2006). When what you say about others says something about you: Language abstraction and inferences about describers' attitudes and goals. Journal of Experimental Social Psychology, 42 (4), 500-8.

Douglas, K., Sutton, R., \& Wilkin, K. (2008). Could you mind your language? An investigation of communicators' ability to inhibit linguistic bias. Journal of Language and Social Psychology, 27(2), 123-139.

Douglas, K. M., \& Sutton, R. M. (2010). By their words ye shall know them: Language abstraction and the likeability of describers. European Journal of Social Psychology, 40(2), 366-374.

Duan, W., Gu, B., \& Whinston, A. B. (2008). The dynamics of online word-of-mouth and product sales--An empirical investigation of the movie industry. Journal of Retailing, 84(2), 233-242. 
East, R., Hammond, K., \& Wright, M. (2007). The relative incidence of positive and negative word of mouth: A multi-category study. International Journal of Research in Marketing, 24(2), 175-184.

Epley, N. \& Caruso, E. M. (2008). Perspective taking: Misstepping into others' shoes. In K. D. Markman, W. M. P. Klein, \& J. A. Suhr (Eds.), The handbook of imagination and mental simulation (pp. 295-309). New York: Psychology Press.

Escalas, J., \& Luce, M. (2003). Process versus outcome thought focus and advertising. Journal of Consumer Psychology, 13(3), 246-254.

Escalas, J., \& Luce, M. (2004). Understanding the effects of process-focused versus outcome-focused thought in response to advertising. Journal of Consumer Research, 31(2), 274-285.

Feick, L., \& Price, L. (1987). The market maven: A diffuser of marketplace information. Journal of Marketing, 51(1), 83-97.

Festinger, L. (1964). Conflict, decision, and dissonance. Stanford: CA: Stanford University Press.

Fiedler, K. (2008a). Language: A toolbox for sharing and influencing social reality. Perspectives on Psychological Science, 3(1), 38-47.

Fiedler, K. (2008b). The implicit meta-theory that has inspired and restricted LCM research. Journal of Language and Social Psychology, 27(2), 182-196.

Fiske, S. T., \& Neuberg, S. L. (1990). A continuum of impression formation, from category-based to individuating processes: Influences of information and motivation on attention and interpretation. Advances in Experimental Social Psychology, 23, 1-74.

Fitzsimons, G., \& Kay, A. (2004). Language and interpersonal cognition: Causal effects of variations in pronoun usage on perceptions of closeness. Personality and Social Psychology Bulletin, 30(5), 547-557.

Franco, F., \& Maass, A. (1996). Implicit versus explicit strategies of out-group discrimination - The role of intentional control in biased language use and reward allocation. Journal of Language and Social Psychology, 15(3), 335-359.

Franco, F., \& Maass, A. (1999). Intentional control over prejudice: When the choice of the measure matters. European Journal of Social Psychology, 29(4), 469-477.

Frenzen, J., \& Nakamoto, K. (1993). Structure, cooperation, and the flow of marketinformation. Journal of Consumer Research, 20(3), 360-375. 
Frey, D. (1986). Recent research on selective exposure to information. Advances in Experimental Social Psychology, 19, 41-76.

Friestad, M., \& Wright, P. (1994). The persuasion knowledge model - How people cope with persuasion attempts. Journal of Consumer Research, 21(1), 1-31.

Fussell, S., \& Krauss, R. (1989). The Effects of Intended Audience on Message Production and Comprehension - Reference in a Common Ground Framework. Journal of Experimental Social Psychology, 25(3), 203-219.

Gilly, M., Graham, J., Wolfinbarger, M., \& Yale, L. (1998). A dyadic study of interpersonal information search. Journal of the Academy of Marketing Science, 26(2), 83-100.

Godes, D., \& Mayzlin, D. (2004). Using online conversations to study word-of-mouth communication. Marketing Science, 23, 545-560.

Godes, D., et al. (2005). The firm's management of social interactions. Marketing Letters, 16(3-4), 415-428.

Goldenberg, J., Libai, B., Moldovan, S., \& Muller, E. (2007). The NPV of bad news. International Journal of Research in Marketing, 24(3), 186-200.

Grice, H. (1975). Logic and conversation. New York: Academic Press.

Guerin, B. (2003). Language use as social strategy: A review and an analytic framework for the social sciences. Review of General Psychology, 7(3), 251-298.

Hamilton, R. (2003). Why do people suggest what they do not want? Using context effects to influence others' choices. Journal of Consumer Research, 29, 492-506.

Hamilton, R., \& Thompson, D. (2007). Is there a substitute for direct experience? Comparing consumers' preferences after direct and indirect product experiences. Journal of Consumer Research, 34(4), 546-555.

Hennig-Thurau, T., Gwinner, K., Walsh, G., \& Gremler, D. (2004). Electronic word-ofmouth via consumer-opinion platforms: What motivates consumers to articulate themselves on the internet? Journal of Interactive Marketing, 18, 38-52.

Herr, P., Kardes, F., \& Kim, J. (1991). Effects of word-of-mouth and product-attribute information on persuasion - an accessibility-diagnosticity perspective. Journal of Consumer Research, 17(4), 454-462. 
Higgins, E. (1981). The "communication game": Implications for social cognition and persuasion. In E. T. Higgins, C. P. Herman, \& M. P. Zanna (Eds.), Social Cognition: The Ontario Symposium (Vol. 1, pp. 343-392). Hillsdale, NJ: Erlbaum.

Higgins, T., \& Rholes, W. (1978). "Saying is Believing": Effect of Messages Modification on Memory and Liking for the Person Described. Journal of Experimental Social Psychology, 14, 363-378.

Holtgraves, T. M., \& Kashima, Y. (2008). Language, meaning, and social cognition. Personality and Social Psychology Review, 12(1), 73-94.

Jain, S. P., \& Maheswaran, D. (2000). Motivated reasoning: A depth-of-processing perspective. Journal of Consumer Research: An Interdisciplinary Quarterly, 26(4), 358-71.

Jain, S. P., \& Posavac, S. (2001). Prepurchase attribute verifiability, source credibility, and persuasion. Journal of Consumer Research, 11(3), 169-80.

Juster, F. T. (1966). Consumer Buying Intentions and Purchase Probability: An Experiment in Survey Design. Journal of the American Statistical Association, 61, 658-96

Kardes, F., Kim, J., \& Lim, J. (1994). Moderating effects of prior knowledge on the perceived diagnosticity of beliefs derived from implicit versus explicit product claims. Journal of Business Research, 29(3), 219-224.

Katz, E. \& Lazarsfeld, P. F. (1955). Personal Influence: The Part Played by People in the Flow of Mass Communication. New York: Free Press.

Keller, E. (2007). Unleashing the power of word of mouth: Creating brand advocacy to drive growth. Journal of Advertising Research, 47 (4), 448-452.

Keller, P. A., \& Block, L. G. (1997). Vividness effects: A resource-matching perspective. Journal of Consumer Research, 24(3), 295-304.

Kim, K., \& Meyers-Levy, J. (2007). Context effects in diverse-category brand environments; The influence of target product positioning and consumers' processing mind-set. Journal of Consumer Research, 34, 882-896

Kim, H., Rao, A. R., \& Lee, A. Y. (2009). It's time to vote: the effect of matching message orientation and temporal frame on political persuasion. Journal of Consumer Research: An Interdisciplinary Quarterly, 35(6), 877-889. 
Kim, J., Kardes, F. R. \& Herr, P. (1991). Consumer expertise and the vividness effect implications for judgement and inference. Advances in Consumer Research, 18, 90-93.

Kisielius, J., \& Sternthal, B. (1984). Detecting and explaining vividness effects in attitudinal judgments. Journal of Marketing Research, 54-64.

Kozak, M., Marsh, A., \& Wegner, D. (2006). What do I think you're doing? Action identification and mind attribution. Journal of Personality and Social Psychology, 90(4), 543-555.

Krishna, A. \& Ahluwalia, R. (2008). Language choice in advertising to bilinguals: asymmetric effects for multinationals versus local firms. Journal of Consumer Research, 35(4), 692-705.

Klucharev, V., Smidts, A., \& Fernandez, G. (2008). Brain mechanisms of persuasion: how 'expert power' modulates memory and attitudes. Social Cognitive and Affective Neuroscience, 3(4), 353-366.

Kruger, J., Epley, N., Parker, J., \& Ng, Z. (2005). Egocentrism over E-mail: Can people communicate as well as they think? Journal of Personality and Social Psychology, $89,925-936$.

Laczniak, R., DeCarlo, T., \& Ramaswami, S. (2001). Consumers' response to negative word-of-mouth communication: An attribution theory perspective. Journal of Consumer Psychology, 11(1), 57-73.

Lee, A., \& Aaker, J. (2004). Bringing the frame into focus: The influence of regulatory fit on processing fluency and persuasion. Journal of Personality and Social Psychology, 86(2), 205-218.

Lee, L., \& Ariely, D. (2006). Shopping goals, goal concreteness, and conditional promotions. Journal of Consumer Research, 33(1), 60-70.

Lee, A. Y., Keller, P. A., \& Sternthal, B. (2010). Value from regulatory construal fit: The persuasive impact of fit between consumer goals and message concreteness. Journal of Consumer Research, 36(5), 735-747.

Liberman, N., \& Trope, Y. (1998). The role of feasibility and desirability considerations in near and distant future decisions: A test of temporal construal theory. Journal of Personality and Social Psychology, 75(1), 5-18.

Liu, Y. (2006). Word of mouth for movies: Its dynamics and impact on box office revenue. Journal of Marketing, 70(3), 74-89. 
Lowrey, T. M. (2007). Psycholinguistic phenomena in marketing communications. Lawrence Erlbaum Associates: NJ.

Luna, D., \& Peracchio, L. A. (2001). Moderators of language effects in advertising to bilinguals: A psycholinguistic approach. Journal of Consumer Research, 28(2), 284-295.

Maass, A., Ceccarelli, R., \& Rudin, S. (1996). Linguistic intergroup bias: Evidence for in-group-protective motivation. Journal of Personality and Social Psychology, 71(3), 512-526.

Maass, A., Karasawa, M., Politi, F., \& Suga, S. (2006). Do verbs and adjectives play different roles in different cultures? A cross-linguistic analysis of person representation. Journal of Personality and Social Psychology, 90(5), 734-750.

Maass, A., Milesi, A., Zabbini, S., \& Stahlberg, D. (1995). Linguistic intergroup bias Differential expectancies or in-group protection. Journal of Personality and Social Psychology, 68(1), 116-126.

Maass, A., Montalcini, F., \& Biciotti, E. (1998). On the (dis-)confirmability of stereotypic attributes. European Journal of Social Psychology, 28(3), 383-402.

Maass, A., Salvi, D., Arcuri, L., \& Semin, G. (1989). Language use in intergroup contexts - The linguistic intergroup bias. Journal of Personality and Social Psychology, 57(6), 981-993.

Mackenzie, S. (1986). The Role of Attention in Mediating the Effect of Advertising on Attribute Importance. Journal of Consumer Research, 13, 174-195.

Maheswaran, D., \& Meyers-Levy, J. (1990). The Influence of message framing and issue involvement. Journal of Marketing Research, 27(3), 361-367.

Maheswaran, D., \& Sternthal, B. (1990). The effects of knowledge, motivation, and type of message on ad processing and product judgments. Journal of Consumer Research, 66-73.

Mazzarol, T., Sweeney, J. C., \& Soutar, G. N. (2007). Conceptualizing word. European Journal of Marketing, 41(11/12), 1475 - 1494.

Mangold, W., Miller, F., \& Brockway, G. (1999). Word-of-mouth communication in the service marketplace. Journal of Services Marketing, 13, 73-89.

Meyers-Levy, J., \& Zhu, R. (2007). The influence of ceiling height: The effect of priming on the type of processing that people use. Journal of Consumer Research, 34(2), 174-186. 
Mittal, V., Ross Jr., W. T., \& Baldesare, P. (1998). The asymmetric impact of negative and positive attribute-level performance on overall satisfaction and repurchase intentions. Journal of Marketing, 62(1), 33-47.

Mizerski, R.W. (1982). An attribution explanation of the disproportionate influence of unfavorable information. Journal of Consumer Research, 9 (1), 301-10

Oakes, P. J. (1987). The salience of social categories. In J. C. Turner, M. A. Hogg, P. J. Oakes, S. D. Reicher, \& M. S. Wetherell (Eds.), Rediscovering the social group: A self-categorization theory (pp. 117-141). Oxford, UK: Basil Blackwell.

Oettingen, G. (1995). Positive fantasy and motivation. In P. M. Gollwitzer \& J. A. Bargh (Eds.), Action science: Linking cognition and motivation to behavior. New York: Guilford.

Oliver, R.L. (1997). Satisfaction: A behavioral perspective on the customer. New York: McGraw-Hill.

Pham, L. B., \& Taylor, S. E. (1999). From Thought to Action: Effects of Process-Versus Outcome-Based Mental Simulations on Performance. Personality and Social Psychology Bulletin, 25(2), 250-260.

Price, L. L., Feick, L. F., \& Higie, R. A. (1989). Preference heterogeneity and coorientation as determinants of perceived informational influence. Journal of Business Research, 19(3), 227-242.

Puntoni, S., de Langhe, B., \& van Osselaer, S. M. J. (2009). Bilingualism and the emotional intensity of advertising language. Journal of Consumer Research, 35(6), 1012-1025.

Reitsma-Van Rooijen, M., Semin, G., \& Van Leeuwen, E. (2006). The effect of linguistic abstraction on interpersonal distance. European Journal of Social Psychology.

Reynolds, T. J. \& Whitlark, D.B. (1995). Applying Laddering Data to Communication strategy and Advertising Practice. Journal of Advertising Research, 35 (7/8), 917.

Richins, M. (1983). Negative word-of-mouth by dissatisfied customers: A pilot study. Journal of Marketing, 47(winter), 68-78.

Ross, M., \& Sicoly, F. (1979). Egocentric biases in availability and attribution. Journal of Personality and Social Psychology, 37, 322-336.

Rossiter, J. R., \& Bellman, S. (2005). Marketing communications: Theory and applications. Frenchs Forest, NSW: Pearson Education Australia. 
Rubini, M., \& Sigall, H. (2002). Taking the edge off of disagreement: Linguistic abstractness and self-presentation to a heterogeneous audience. European Journal of Social Psychology, 32(3), 343-351.

Ryu, G., \& Feick, L. (2007). A penny for your thoughts: Referral reward programs and referral likelihood. Journal of Marketing, 71(1), 84-94.

Schau, H., \& Gilly, M. (2003). We are what we post? Self-presentation in personal Web space. Journal of Consumer Research, 30(3), 385-404.

Schmid, J., \& Fiedler, K. (1998). The backbone of closing speeches: The impact of prosecution versus defense language on judicial attributions. Journal of Applied Social Psychology, 28(13), 1140-1172.

Schwarz, N. (1994). Judgment in a social context: Biases, shortcomings, and the logic of conversation. Advances in Experimental Social Psychology, 26, 123-162.

Sekaquaptewa, D., Espinoza, P., Thompson, M., Vargas, P., \& Von Hippel, W. (2003). Stereotypic explanatory bias: Implicit stereotyping as a predictor of discrimination. Journal of Experimental Social Psychology, 39(1), 75-82.

Semin, G. (1994). Linguistic category model and personality language. In The status of common sense in psychology (pp. 305-321). Plenum Press.

Semin, G. (2000). Agenda 2000 - Communication: Language as an Implementational device for cognition. European Journal of Social Psychology, 30(5), 595-612.

Semin, G., \& Fiedler, K. (1988). The cognitive functions of linguistic categories in describing persons - social cognition and language. Journal of Personality and Social Psychology, 54(4), 558-568.

Semin, G., \& Fiedler, K. (1989). Relocating attributional phenomena within a languagecognition interface - the case of actors and observers perspectives. European Journal of Social Psychology, 19(6), 491-508.

Semin, G., \& Fiedler, K. (1991). The linguistic category model, its bases, applications and range. European Review of Social Psychology, 203, 1-30.

Semin, G., Higgins, T., de Montes, L., Estourget, Y., \& Valencia, J. (2005). Linguistic signatures of regulatory focus: How abstraction fits promotion more than prevention. Journal of Personality and Social Psychology, 89(1), 36-45.

Semin, G., Gil de Montes, L., \& Valencia, J. (2003). Communication constraints on the linguistic intergroup bias. Journal of Experimental Social Psychology, 39(2), 142148. 
Sherman, J. W., Lee, A. Y., Bessenoff, G. R., \& Frost, L. A. (1998). Stereotype efficiency reconsidered: Encoding flexibility under cognitive load. Journal of Personality and Social Psychology, 75, 589-606.

Shiv, B., Britton, J., \& Payne, J. (2004). Does elaboration increase or decrease the effectiveness of negatively versus positively framed messages? Journal of Consumer Research, 31(1), 199-208.

Smith, R., \& Vogt, C. (1995). The effects of integrating advertising and negative wordof-mouth communications on message processing and response. Journal of Consumer Psychology, 4(2), 133-151.

Sperber, D., \& Wilson, D. (1995). Relevance (2nd ed.). Cambridge, UK: Cambridge University Press.

Stapel, D., \& Semin, G. (2007). The magic spell of language: Linguistic categories and their perceptual consequences. Journal of Personality and Social Psychology, 93(1), 23-33.

Sundaram, D. S., Mitra, K., \& Webster, C. (1998). Word-of-mouth communications: A motivational analysis. Advances in Consumer Research, 25, 527-531.

Tajfel, H. and Turner, J. C. (1986). The social identity theory of inter-group behavior. In S. Worchel and L. W. Austin (eds.), Psychology of Intergroup Relations. Chicago: Nelson-Hall.

Taylor, S. E., Pham, L. B., Rivkin, I. D., \& Armor, D. A. (1998). Harnessing the imagination: Mental simulation, self-regulation, and coping. American Psychologist, 53(4), 429-439.

Thurau, T., Gwinner, K., Walsh, G., \& Gremler, D. (2004). Electronic word-of-mouth via consumer-opinion platforms: What motivates consumers to articulate themselves on the internet? Journal of Interactive Marketing, 18, 38-52.

Trope, Y., Liberman, N., \& Wakslak, C. (2007). Construal levels and psychological distance: Effects on representation, prediction, evaluation, and behavior. Journal of Consumer Psychology, 17(2), 83-95.

Tuk, M. A., Verlegh, P. W., Smidts, A., \& Wigboldus, D. H. (2009). Sales and sincerity: The role of relational framing in word-of-mouth marketing. Journal of Consumer Psychology, 19(1), 38-47. 
Vallacher, R. R., \& Wegner, D. M. (1989). Levels of personal agency: Individual variation in action identification. Journal of Personality and Social Psychology, 56(2), 199-208.

Van der Lans, R., et al. (2009). Cross-national logo evaluation analysis: An individuallevel approach. Marketing Science, 28(5), 968-985.

Van Hoye, G., \& Lievens, F. (2007). Social influences on organizational attractiveness: investigating if and when word of mouth matters. Journal of Applied Social Psychology, 37(9), 2024-2047.

Van Hoye, G., \& Lievens, F. (2009). Tapping the grapevine: A closer look at word-ofmouth as a recruitment source. Journal of Applied Psychology, 94(2), 341-352.

Von Hippel, W., Sekaquaptewa, D., \& Vargas, P. (1997). The Linguistic Intergroup Bias as an Implicit Indicator of Prejudice. Journal of Experimental Social Psychology, 33(5), 490-509.

Watts, D., \& Dodds, P. (2007). Influentials, networks, and public opinion formation. Journal of Consumer Research, 34(4), 441-458.

Webster, D., Kruglanski, A., \& Pattison, D. (1997). Motivated language use in intergroup contexts: Need-for-closure effects on the linguistic intergroup Bias. Journal of Personality and Social Psychology, 72(5), 1122-1131.

Wenneker, C., Wigboldus, D., \& Spears, R. (2005). Biased language use in stereotype maintenance: The role of encoding and goals. Journal of Personality and Social Psychology, 89(4), 504-516.

Westbrook, R.A. (1987). Product/consumption-based affective responses and postpurchase process. Journal of Marketing Research, 24(3), 258-270.

White, K., \& Dahl, D. (2007). Are all out-groups created equal? Consumer identity and dissociative influence. Journal of Consumer Research, 34(4), 525-536.

Wigboldus, D., \& Douglas, K. (2007). Language, stereotypes, and intergroup relations. In K. Fiedler (Ed.), Social Communication (pp. 79-106). New York and Hove: Psychology Press.

Wigboldus, D., Semin, G., \& Spears, R. (2000). How do we communicate stereotypes? Linguistic bases and inferential consequences. Journal of Personality and Social Psychology, 78(1), 5-18.

Wigboldus, D., Semin, G., \& Spears, R. (2006). Communicating expectancies about others. European Journal of Social Psychology, 36(6), 815-824. 
Wigboldus, D., Spears, R., \& Semin, G. (2005). When do we communicate stereotypes? Influence of the social context on the linguistic expectancy bias. Group Processes E Intergroup Relations, 8(3), 215-230.

Wirtz, J., \& Chew, P. (2002). The effects of incentives, deal proneness, satisfaction and tie strength on word-of-mouth behaviour. International Journal of Service Industry Management, 13, 141-162.

Wright, M., \& MacRae, M. (2007). Bias and variability in purchase intention scales. Journal of the Academy of Marketing Science, 35(4), 617-24.

Zhao, M., Hoeffler, S., \& Zauberman, G. (2007). Mental simulation and preference consistency over time: The role of process- versus outcome-focused thoughts. Journal of Marketing Research, 44(3), 379-388. 



\section{Executive Summary}

This dissertation highlights the importance of understanding how consumers frame product messages, and what impact their language has on receivers of word of mouth. A main property of language is its abstractness, and language abstraction is a valuable and useful communication signal which can be used by communicators to optimize their interactions (Semin \& Fiedler, 1988; Semin, 2000). Research in social psychology found a systematic pattern in the use of language abstraction in interpersonal communication (Maass, Salvi, Arcuri, \& Semin, 1989; Wigboldus, Spears, \& Semin, 2000). This dissertation not only examined how consumers vary language abstractness in word of mouth, but also how language abstraction affects the receiver of a message.

The present dissertation is the first to examine language abstraction outside the interpersonal domain and to evaluate language use in descriptions of products. Chapter 2 reveals that product experiences that are congruent with the product attitude of the sender are communicated more abstractly, than attitude incongruent experiences. Chapter 3 shows that senders use language abstraction adaptively by adjusting to a communication situation, such as to the goal of the communication or to the characteristics of the receiver of the message. Chapter 4 demonstrates that language abstraction can have an impact on receivers. Receivers are shown to be able to infer a senders' product attitude from the abstractness of a senders' product message. Moreover, a positive recommendation framed abstractly is shown to induce a higher purchase intention than the same recommendation that is worded concretely. Whereas, negative word of mouth framed concretely has a less negative effect on the purchase intention than an abstract version. This process is also shown to be moderated by the mindset of the receiver. The valence of messages is excluded as a possible alternative explanation for the use of abstract versus concrete language, and for the impact of language abstraction on receivers.

This research is of interest to marketers for two reasons. First, these findings may help firms to interpret (online) word of mouth. By analyzing the language abstraction that is used in word of mouth, firms may determine the product attitude of consumers. Secondly, the effect of language abstraction on receivers can be of use to marketers who are seeking to manage and optimize word-of-mouth marketing (Ryu \& Feick, 2007). Future research should examine whether the findings can be extended to marketing communication in general. 



\section{Summary (Dutch)}

In het dagelijks leven praten mensen regelmatig over hun ervaringen met producten en diensten. Deze conversaties staan ook wel bekend als mond-tot-mond communicatie en zijn een belangrijke bron van informatie voor consumenten die op het punt staan een product aan te schaffen. Mond-tot-mond communicatie is een van de oudste methoden om informatie over diensten en producten te verspreiden en heeft sinds het invloedrijke werk van Katz and Lazarsfeld's (1955) doorlopend aandacht gekregen van onderzoekers (Arndt, 1967; Brown \& Reingen, 1987; Chevalier \& Mayzlin, 2006; Frenzen \& Nakamoto, 1993; Watts \& Dodds, 2007).

Er zijn verschillende manieren waarop consumenten hun ervaringen en beoordelingen van producten en diensten met elkaar kunnen delen. Zo kan iemand een negatieve ervaring met een T-shirt dat na één keer wassen zijn kleur verliest, beschrijven als 'mijn shirt is verkleurd', maar deze persoon kan ook zeggen 'mijn shirt is van slechte kwaliteit'. In het eerste geval geeft deze persoon een concrete beschrijving van de ervaring, terwijl hij in het laatste geval een abstracte verwoording gebruik die de ervaring generaliseert naar een algemene indruk van de kwaliteit van het T-shirt. Dit proefschrift laat in een serie van experimenten zien wanneer en waarom consumenten concrete en abstracte taal gebruiken en hoe dit taalgebruik de ontvanger van de boodschap beïnvloedt.

Taalabstractie is een belangrijk onderdeel van taal. De abstractheid van taal stelt iemand in staat eenzelfde gebeurtenis te beschrijven op uiteenlopende manieren en dit reflecteert de beoogde boodschap van de zender (Semin \& Fiedler, 1988, 1989, 1991). Zodoende kan de taalabstractie van een boodschap informatie onthullen over psychologische en sociale processen (Maass, et al., 1995; Semin \& Fiedler, 1988). Het kan ook een belangrijke rol spelen in het waarnemen van informatie (Semin, 2000; Holtgrave \& Kashima, 2008; Wigboldus, et al., 2000), omdat het, onafhankelijk van de inhoud van de boodschap, de aandacht van een ontvanger richt op een bepaald facet van de beoogde boodschap. Taalabstractie vormt daarmee de cognitieve processen van de ontvanger.

Taalabstractie wordt volgens het linguïstisch categorieën model (Semin \& Fiedler, 1988) ingedeeld in vier niveaus, welke lopen van concreet (descriptieve actie werkwoorden; DAV's) via tussenliggende niveaus (interpretatieve actie werkwoorden: IAV's en toestandswoorden; SV) naar abstract (adjectieven; Adj's). Voorbeelden van 
deze vier niveaus zijn: "Jan slaat Kees” (DAV), “Jan pijnigt Kees” (IAV), “Jan haat Kees" (SV) en "Jan is agressief" (Adj). Een meer abstracte beschrijving van gedrag wekt de indruk dat de gebeurtenis bepaald wordt door een typische en stabiele karaktereigenschap van de handelende persoon, terwijl een meer concrete beschrijving de aandacht richt op de specifieke situatie waarin het gedrag plaatsvindt (Semin \& Fiedler, 1988). Een concrete beschrijving focust op de eenmalige gebeurtenis in een specifieke context en generaliseert de informatie niet naar de persoon. Het is hierbij van belang op te merken dat taalabstractie volgens het linguïstische categorieën model niet vergelijkbaar is met eerder marketing onderzoek naar concreet- en abstractheid (bv. Aggarwal \& Law, 2005; Corfman, 1991; Hamilton \& Thompson, 2007; Keller \& Block, 1997; Kim \& Meyers-Levy, 2007; Kim, Rao, \& Lee, 2007; Lee \& Ariely, 2006; Lee, Keller \& Sternthal, 2010; Mackenzie, 1986; Maheswaran \& Sternthal, 1990; MeyersLevy \& Zhu, 2007; Zhao, Hoeffler \& Zauberman, 2007). In marketing onderzoek werden concreet- en abstractheid gemanipuleerd op basis van de betekenis van de informatie (bv. concrete informatie is abstracte informatie met toevoeging van feiten), terwijl het in het linguïstisch categorieën model draait om de vorm waarin de informatie beschreven wordt (het gebruik van werkwoorden en adjectieven).

Eerder onderzoek in de sociale psychologie heeft aangetoond dat de taalabstractie die mensen gebruiken om menselijk gedrag te beschrijven, wordt beïnvloed door de mate waarin dit gedrag overeenkomt met de verwachtingen van de beschrijver (Douglas \& Sutton, 2003; Maass, Salvi, Arcuri, \& Semin, 1989; Maass, Milesi, Zabbini, \& Stahlberg, 1995; Von Hippel, Sekaquaptewa, \& Vargas, 1997; Webster, Kruglanski, \& Dwight 1997; Wigboldus, Spears, \& Semin, 2000). Deze verwachtingen kunnen gebasseerd zijn op stereotypen over of groepslidmaatschap van de handelende persoon. Zo wordt van groepsgenoten verwacht dat ze positieve gedragingen vertonen en wordt van buitenstaanders (outgroupmembers) eerder ongewenst gedrag verwacht. Deze veronderstellingen worden verwoord in abstacte taal ('onze groepsgenoot is behulpzaam' en 'onze tegenstander is agressief'). Daarentegen zijn ongewenste handelingen van groepsgenoten en positief gedrag van buitenstaanders onverwacht en worden deze meer concreet beschreven ('onze groepsgenoot duwt iemand' en 'onze tegenstander geeft iemand een hand'). Dit stelselmatig gebruik van taalabstractie werd gelabeld als linguïstische verwachtingsvertekening ('linguïstic expectancy bias') en linguïstische tussengroepsvertekening ('linguïstic intergroup bias'). Sociaal psychologisch onderzoek naar taalabstractie beperkt zich grotendeels tot communicatie over 
(inter)persoonlijk gedrag, terwijl mensen ook frequent communiceren over objecten en producten (mond-tot-mond communicatie). In dit proefschrift wordt het gebruik van taalabstractie in mond-tot-mond communicatie en de effecten van de abstractheid van taal op ontvangers van product referenties bestudeerd om meer inzicht te krijgen in het fenomeen mond-tot-mond communicatie.

\section{Effect van de Product Attitude van de Zender op Taalabstractie in Mond-tot-Mond Communicatie}

Het eerste empirische hoofdstuk (hoofdstuk 2) laat zien op welke manier de taalabstractie van een productbeschrijving wordt bepaald door de mate waarin een productervaring in overeenstemming is met de productattitude (mening) van de spreker. In meerdere experimenten werden producten van een fictief of bestaand merk gebruikt of getest door een proefpersoon of zag de proefpersoon een andere consument dit product gebruiken (vertoond op video). Hierna werden de proefpersonen gevraagd de ervaring te beschrijven aan iemand anders. Hieruit bleek dat proefpersonen een positieve ervaring met een product (bv. gemakkelijk glad scheren met een nieuw scheermes) abstract beschreven wanneer zij vóór het gebruik van het product al een positieve attitude hadden over dit product. Met een positieve attitude worden positieve ervaringen gezien als typisch en representatief en abstract taalgebruik benadrukt dit. Wanneer proefpersonen een negatieve attitude hadden, beschreven zij een positieve ervaring echter concreet. Concreet taalgebruik helpt deze ervaring te omschrijven als een eenmalige situatie, geïsoleerd van het merk in zijn geheel. Bovendien werd ook gevonden dat wanneer proefpersonen een negatieve attitude hadden over een product, zij een negatieve ervaring met een product abstract beschreven, terwijl zij dezelfde negatieve ervaring met een favoriet merk concreet omschreven. Consumenten beschrijven productervaringen dus verschillend, afhankelijk van hun eigen attitude over dit product, waarbij verwachtingscongruente productervaringen abstracter worden beschreven dan verwachtingsincongruente ervaringen. Een serie van 6 experimenten met circa 700 proefpersonen met verschillende manipulaties voor onafhankelijke en afhankelijke variabelen en een grote verscheidenheid aan productcategorieën (van diensten tot elektronica en 'fast moving consumer goods') dragen bij aan de robuustheid van mijn bevindingen over stelselmatig gebruik van taalabstractie in mond-tot-mond communicatie. 
Een tweede essentiële contributie van hoofdstuk 2 is de aanpak van een belangrijk kritiekpunt van eerder onderzoek naar taalabstractie, namelijk de inherente relatie tussen de taalabstractie van een beschrijving en de positiviteit of negativiteit van die beschrijving ('valence', hierna: valentie). Bijvoorbeeld, mijn T-shirt is van slechte kwaliteit' kan gezien worden als een meer negatieve beschrijving dan 'mijn Tshirt is verkleurd'. Dit proefschrift toonde aan dat de valentie van een boodschap niet verantwoordelijk is voor het stelselmatige gebruik van taalabstractie in mond-totmond communicatie, noch voor de effecten van taalabstractie op een ontvanger. Verscheidene experimenten van hoofdstuk 2 lieten consequent zien dat de valentie van productbeschrijvingen niet de effecten van productattitude en productervaring op gebruik van taalabstractie kunnen verklaren (bv. experimenten 2.1 en 2.5). Van groot belang is experiment 2.2 welke experimenteel de samenhang tussen de taalabstractie en de valentie van een beschrijving manipuleerde. Hierdoor werd het volledig duidelijk dat systematische variatie in taalabstractie niet gedreven werd door de valentie van concrete en abstracte taal.

Het derde doel van hoofdstuk 2 was het verkennen van het onderliggende mechanisme van stelselmatig gebruik van taalabstractie in mond-tot-mond communicatie. Hoewel linguïstische verwachtings- en tussengroepsvertekening algemeen erkende begrippen zijn (Maass, et al., 1989, 1995, 1996; Sekaquaptewa et al., 2003; Webster, Kruglanski, \& Dwight, 1997), is er nog relatief weinig bekend over het exacte onderliggende mechanisme van deze fenomenen. Het laatste experiment van hoofdstuk 2 liet zien dat stelselmatig taalgebruik in mond-tot-mond communicatie wordt veroorzaakt doordat verwachtingscongruente productervaringen werden toegeschreven aan de intrinsieke eigenschappen van het merk en niet aan de situatie of de specifieke gebruiker. Bijvoorbeeld, met een positieve attitude over een merk wordt een positieve ervaring met dit merk gezien als veroorzaakt door de eigenschappen van het merk (attributie aan het merk). Daarentegen werden onverwachte productervaringen aan de gebruiker of situatie gewijd en niet aan het merk.

De bevindingen van hoofdstuk 2 kunnen gebruikt worden door marketeers om meer inzicht te krijgen in de mond-tot-mond communicatie van hun klanten. Tegenwoordig bestuderen veel bedrijven (voornamelijk online) mond-tot-mond communicatie en proberen ze te leren van deze informatiestroom die beschikbaar is in blogs en (online) product reviews. Het onderscheiden van concreet en abstract taalgebruik zou verdieping kunnen aanbrengen in deze analyses en marketeers kunnen helpen om verder te gaan dan het registreren van de hoeveelheid en verdeling 
van positieve en negatieve reviews (bv. Godes \& Mayzlin, 2004; Liu, 2006). Door het analyseren van de taalabstractie in de productbeschrijvingen van consumenten zouden marketeers (of onderzoekers in het algemeen) de onderliggende productattitude van de schrijver bloot kunnen leggen. Het kan hen helpen onderscheid te maken tussen consumenten die een negatieve ervaring als een eenmalig afzonderlijke gebeurtenis ervaren en consumenten die deze ervaring zien als een bevestiging van hun negatieve verwachtingen. Zo kan men uit een concreet beschreven ongunstige review afleiden dat de zender over het algemeen positief is over het product of merk. Klanten service medewerkers of webcare teams zouden in het bijzonder veel aandacht dienen te schenken aan klanten die in concrete taal klagen, omdat er bij deze klanten een grote kans is dat zij na een effectieve klachtenafhandeling wederom tevreden zijn.

\section{Variatie in Taalabstractie door Communicatiesituatie}

Eerder onderzoek heeft laten zien dat mensen zich niet bewust zijn van het niveau van taalabstractie waarop zij communiceren, noch zijn zij op de hoogte van de aanleiding voor een bepaald niveau van taalabstractie (Maass et al., 1995; Franco \& Maass, 1996, 1999; Von Hippel, Sekaquaptewa, \& Vargas, 1997; Webster, Kruglanski, \& Pattison, 1997). Desalniettemin laat een aantal studies zien dat taalabstractie strategisch gebruikt kan worden om bijvoorbeeld groepsgenoten te beschermen (Maass et al., 1996, 1998; Sekaquaptewa et al., 2003; Schmid \& Fiedler, 1998), om cognitieve duidelijkheid ('closure') te bereiken (Webster, Kruglanski, \& Pattison, 1997), of in reactie op communicatie instructies (Douglas \& Sutton, 2003; Douglas, Sutton, \& Wilkin, 2008; Semin, et al., 2005; Rubini \& Sigall, 2002; Wenneker, et al., 2005).

Ter uitbreiding van dit onderzoek kijkt hoofdstuk 3 van dit proefschrift naar de invloed van de communicatiesituatie op taalabstractie in mond-tot-mond communicatie. In dit hoofdstuk wordt beargumenteerd dat de taalabstractie die consumenten gebruiken in mond-tot-mond communicatie niet alleen afhankelijk is van de productattitudes van een zender, maar wellicht ook van belangrijke facetten in de communicatiesituatie: de ontvanger (en zijn kenmerken) en het doel waarmee iemand communiceert. Waar in eerder onderzoek naar de invloed van communicatiedoelen op taalabstractie de aandacht van de proefpersonen werd gericht op de taalabstractie van het stimulusmateriaal (bv. Douglas \& Sutton, 2003), wordt in het huidige onderzoek gekeken naar de invloed van de huidige en gewenste productattitude van ontvangers op het gebruik van taalabstractie van de zender. 
De mogelijkheid om een boodschap aan te passen aan de communicatiesituatie is een van de pijlers van mond-tot-mond communicatie. Daarom is het aannemelijk dat consumenten hun gebruik van taalabstractie kunnen aanpassen aan de karakteristieken van een ontvanger. Hoofdstuk 3 van dit proefschrift toonde aan dat consumenten meer abstracte taal gebruikten voor informatie die congruent was met de productattitude van de ontvanger. Met andere woorden, een negatieve productervaring werd concreter verwoord tegen iemand met een positieve (vergeleken met een negatieve) productattitude, terwijl een positieve ervaring abstracter werd gecommuniceerd tegen iemand met een positieve (vergeleken met een negatieve) productattitude. Voor vervolgonderzoek is het interessant te kijken naar het effect van de relatie tussen zender en ontvanger op taalabstractie. Bijvoorbeeld, in communicatie binnen een zwakke relatie ('weak tie'; Brown \& Reingen, 1987; Van Hove \& Lievens, 2009) zal men abstracte productclaims wellicht vermijden, omdat men niet op de hoogte is van de productattitude van de ontvanger.

Daarnaast zijn in hoofdstuk 3 de effecten getoetst van een communicatiedoel van de zender op het gebruik van taalabstractie. Consumenten zouden de abstractheid van taal kunnen gebruiken om de gedachten en gevoelens van ontvangers te beïnvloeden en de aandacht van ontvangers te richten op een bepaald facet van de realiteit: meer abstracte taal benadrukt de algemene en stabiele kwaliteiten van een product. In Hoofdstuk 3 werd gevonden dat consumenten die tot doel hadden een ontvanger te overtuigen van de hoge kwaliteit van een product, positieve productervaringen meer abstract beschreven en negatieve productervaringen meer concreet, dan wanneer zij dezelfde informatie beschreven zonder overtuigingsdoel. Hoewel iemand overtuigen een belangrijk en veelvoorkomend communicatiedoel is, zou vervolgonderzoek zich kunnen richten op effecten van andere communicatiedoelen. Bijvoorbeeld, het doel om jezelf positief te positioneren ('self enhancement goal') activeert waarschijnlijk meer abstracte taal in beschrijvingen van positief gedrag.

Deze bevindingen kunnen interessant zijn voor marketeers die de communicatie over hun product of merk willen beïnvloeden. Door het activeren van een communicatiedoel in reclame of direct marketing kan de wenselijke mond-totmond communicatie in het gewenste niveau van taalabstractie worden bereikt. Bijvoorbeeld, bedrijven zouden hun eigen klanten kunnen stimuleren aanbevelingen te doen aan andere consumenten (bv. met cadeautjes of korting; Biyalogorsky, Gerstner, \& Libai, 2001; Rye \& Feick, 2007). Omdat eigen klanten over het algemeen een 
positieve productattitude hebben, zullen zij positieve ervaringen abstract communiceren. Een andere mogelijkheid om het gewenste niveau van taalabstractie in mond-tot-mond communicatie te verkrijgen is door concreet dan wel abstract taalgebruik te activeren of stimuleren (Stapel \& Semin, 2007).

\section{Effecten van Taalabstractie op Ontvangers}

Eerder onderzoek naar taalabstractie was voornamelijk gericht op de factoren die het taalgedrag van zenders beïnvloeden. Veel minder was er bekend over de invloed van taalabstractie op ontvangers. In dit proefschrift wordt taalabstractie verondersteld als een communicatiesignaal. Voorbijgaand aan de inhoud en de valentie (positiefnegatief) van een boodschap zouden ontvangers subtiele aanwijzingen verborgen in taalabstractie kunnen signaleren en erdoor beïnvloed kunnen worden. De bevindingen van hoofdstuk 3 suggereren dat consumenten zich impliciet bewust zijn van de effecten van taalabstractie op ontvangers. Hoofdstuk 4 onderzoekt de werkelijke effecten van taalabstractie. Dit hoofdstuk is waarschijnlijk de belangrijkste contributie van dit proefschrift, omdat het een essentiële bijdrage levert aan de literatuur over taalgebruik, en relevant en praktisch toepasbaar is voor marketeers.

Ten eerste laat hoofdstuk 4 ter uitbreiding van op basis van eerder empirisch werk (e.g., Douglas \& Sutton, 2006, 2010; Reitsma-Van Rooijen, Semin, \& Van Leeuwen, 2006) zien dat ontvangers informatie over de productattitude van de zender kunnen distilleren uit de taalabstractie van een product boodschap. Hierbij is het van belang te vermelden dat het afleiden van de productattitude van een zender uit een productbeschrijving een ingewikkelde taak is waarin verscheidene motieven en variabelen de inhoud en vorm van deze beschrijving kunnen beïnvloeden (Sundaram, et al., 1998). Ontvangers zijn zich waarschijnlijk bewust van de vele factoren waaraan zij het gebruik van concreet of abstract taalgebruik kunnen toeschrijven. Desondanks werd er in hoofdstuk 4 gevonden dat ontvangers van meer abstracte taal in mond-totmond communicatie concludeerden dat een zender een meer 'extreme' of uitgesproken productattitude heeft. Specifiek betekent dit dat een ontvanger uit een abstract beschreven negatieve ervaring afleidde dat een zender negatiever was dan wanneer deze zender dezelfde ervaring meer concreet beschreef. Terwijl een ontvanger uit een meer abstracte (vergeleken met meer concrete) beschrijving van een positieve ervaring afleidde dat een zender een meer positieve productattitude had. Met andere woorden, een ontvanger bestempelt een zender als meer positief over een 
product wanneer deze positieve mond-tot-mond communicatie abstract beschrijft en negatieve productervaringen concreet beschrijft.

Ten tweede vergrootte dit hoofdstuk onze kennis over de effecten van taalabstractie op de gedragsintenties van ontvangers. Naar mijn mening leveren deze experimenten het eerste bewijs voor de effecten van taalabstractie op de attitude van ontvangers over het beschreven object en hun gedrag in relatie tot dit object (in dit geval: aankoopintentie). Hoofdstuk 4 toonde aan dat eenzelfde positieve aanbeveling leidt tot een hogere aankoopintentie wanneer deze abstract in plaats van concreet werd verwoord. Terwijl een negatieve productbeschrijving in concrete taal tot een minder negatief effect op aankoopintentie leidt dan een negatieve aanbeveling in abstracte taal. Deze effecten bleven significant na correctie voor de invloed van de verschillen in valentie van de concrete en abstracte beschrijvingen. Valentie werd hiermee dus uitgesloten als tussenliggende stap in het effect van taalabstractie op gedragsintentie. Over het algemeen suggereren deze bevindingen dus dat bedrijven het zouden moeten prefereren als consumenten positieve bedrijfs-, product- en merkinformatie abstract formuleren en ongunstige informatie concreet.

Is positieve informatie wanneer zij abstract omschreven wordt dan altijd meer overtuigend dan in concrete taal? Zo simpel is het waarschijnlijk niet. Mond-tot-mond communicatie is een interactief en flexibel proces en dit heeft consequenties voor het taalgebruik van de deelnemende partijen. Een boodschap kan meer of minder aannemelijk zijn afhankelijk van de communicatiesituatie, zoals het doel dat een ontvanger voor ogen heeft met een product (de 'mindset').

De derde bijdrage van hoofdstuk 4 was aantonen hoe de focus ('mindset') van een ontvanger de overredingskracht van taalabstractie beïnvloedt. Het laatste experiment van hoofdstuk 4 liet zien dat een productbeschrijving in concrete taal meer overtuigend is dan in abstracte taal voor een ontvanger die handelingsgericht is ('process mindset': wanneer iemand nadenkt over het gebruik van een product). Daarentegen is een abstracte boodschap meer overtuigend wanneer een ontvanger uitkomstgericht is ('outcome mindset': wanneer iemand nadenkt over het eindresultaat (de gevolgen) van het gebruik van een product). Dit betekent dat een positieve aanbeveling abstract omschreven niet altijd meer overtuigend is, maar dat de overtuigingskracht van taalabstractie afhangt van de communicatiesituatie.

Naast de 'mindset' van een ontvanger zijn er ook andere variabelen in de communicatiesituatie interessant die de overtuigendheid van taalabstractie zouden kunnen beïnvloeden. Voorbeelden zijn de cognitieve capaciteit en (de sterkte van) de 
productattitude van een ontvanger. Dit proefschrift heeft zich beperkt tot het onderzoeken van de effecten van taalabstractie op ontvangers die vooraf geen mening hadden over het product ter discussie ('new brand users'). Consumenten hebben echter dikwijls een mening over producten en diensten (bv. 'other brand loyals'; Rossiter \& Bellman, 2005). Een gevestigde attitude over een merk of product kan invloed hebben op de mate waarin nieuwe informatie over dit product wordt overwogen. In de algemene discussie van dit proefschrift (hoofdstuk 5) worden enkele verwachtingen ten aanzien van de relatie van de attitude van de ontvanger en de invloed van taalabstractie beschreven. Zo wordt bijvoorbeeld voorspeld dat voor informatie die in lijn is met de attitude van de ontvanger (bv. een ontvanger met een positieve attitude krijgt een positieve aanbeveling) de effecten van taalabstractie vergroot zullen zijn, omdat abstracte taal de generaliseerbaarheid van de informatie benadrukt.

\section{Mond-tot-mond Communicatie en Marketingcommunicatie}

Dit proefschrift is gericht op taalabstractie in communicatie tussen consumenten onderling ('consumer-to-consumer' communicatie). Een interessante vraag is of de bevindingen van dit proefschrift ook van toepassing zijn op marketingcommunicatie. Kan een marketeer de resultaten van dit proefschrift toepassen in commerciële communicatie, zoals in reclame en PR? Ik verwacht dat veel van de bevindingen van dit proefschrift van kracht zullen blijven in 'business-to-consumer' communicatie, maar wellicht kunnen zij niet direct gegeneraliseerd worden naar alle vormen van marketingcommunicatie. Een belangrijk verschil tussen mond-tot-mond communicatie en marketingcommunicatie is dat de eerste minder overtuigingskennis ('persuasion knowledge'; Campbell, 1999; Campbell \& Kirmani, 2000, 2008; Friestad \& Wright, 1994) en weerstand activeert. Ik veronderstel dat deze variabelen de overtuigingskracht van taalabstractie zouden kunnen beïnvloeden. Toekomstig onderzoek zal moeten uitwijzen of overtuigingskennis het effect van taalabstractie op ontvangers inderdaad beïnvloedt.

Veel marketingcommunicatie activeert echter geen overtuigingskennis (Campbell \& Kirmani, 2000). Bijvoorbeeld, als consumenten afgeleid zijn ('cognitively constrained'). In deze situaties suggereren de bevindingen van dit proefschrift dat bedrijven het meest positief overkomen in hun communicatie naar consumenten als zij positieve productinformatie abstract beschrijven en negatieve informatie concreet formuleren. Voor het minimaliseren van de impact van negatieve informatie, zou 
Toyota er bijvoorbeeld goed aan doen het terughalen van hun auto's en de storingen van hun producten in concreet taalgebruik te communiceren. Daarnaast moet bij de woordkeuze (taalabstractie) van marketingcommunicatie ook de doelgroep van deze communicatie in overweging worden genomen (zoals 'new category users, brand loyals or other brand loyals/switchers'; Rossiter \& Bellman, 2005).

\section{Conclusies}

Samengevat kijkt dit proefschrift naar het gebruik en de effecten van taalabstractie in mond-tot-mond communicatie. Een serie experimenten laat zien wanneer en waarom consumenten concrete en abstracte taal gebruiken in mond-tot-mond communicatie en hoe verschillen in taalabstractie de ontvanger van mond-tot-mond communicatie kunnen beïnvloeden. Naast een belangrijke contributie aan de literatuur over taalabstractie en mond-tot-mond communicatie, draagt dit proefschrift ook bij aan de groeiende stroom van literatuur over taal en formulering van informatie in consumentengedrag (bv. Jain \& Posovac, 2001; Kim, Rao, \& Lee, 2009; Krishna \& Ahluwalia, 2008; Lee \& Aaker, 2004; Lowrey, 2007; Luna \& Peracchio, 2005; Maheswaran \& Meyers-Levy, 1990; Puntoni, De Langhe, \& Van Osselaer, 2009; Shiv, Britton, \& Payne, 2004).

Mocht er in de toekomst iemand aan $u$ vragen waar mijn onderzoek of dit boek over gaat, dan hoop ik dat $\mathrm{u}$ de volgende twee zaken zult hebben onthouden: 1) consumenten gebruiken meer abstracte taal wanneer zij productervaringen beschrijven die overeenkomen met de mening die zij van tevoren al over het product hadden, en 2) abstracte beschrijvingen hebben over het algemeen een grotere invloed op andere consumenten. 


\section{Acknowledgements (Dutch)}

In informele gesprekken over het verloop van mijn PhD traject kwam ik er snel achter dat 'gaat wel lekker' niet de verwachte response is. Je behoort het zwaar te hebben als PhD en te klagen. Natuurlijk vielen bij mij ook wel eens de uitkomsten van experimenten tegen, had ik verloren onderzoekslijnen en flinke onzekerheden over van alles, maar ik heb ook ontzettend genoten. Een aantal mensen heeft aan mijn vreugde bijgedragen en mij gesteund wanneer ik niet met mijn hoofd in de wolken liep. Deze mensen wil ik in dit dankwoord graag in het zonnetje zetten.

Als voornaamste voor mijn dissertatie, mijn promotoren Ale en Peeter, die mij met hun betrokken, enthousiaste en kritische begeleiding enorm hebben uitgedaagd.

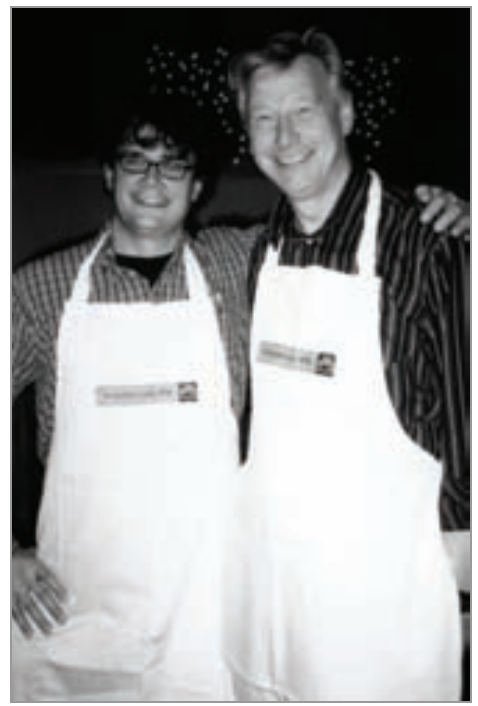

Ale, je hebt me de vrijheid gegeven mijn eigen niche te creëren in het marketing vakgebied waardoor ik mezelf heb kunnen ontwikkelen als een zelfstandig onderzoeker. Ik vond het heerlijk om met je samen te werken. Ongeacht hoe druk je was, je maakte altijd tijd voor me en stak veel energie in het lezen en becommentariëren van mijn stukken. We konden samen flink discussiëren, maar we waren ook vaak eensgezind. Bovenal hebben we ontzettend veel lol gehad: veel van onze discussies eindigden in een lachbui. Bedankt voor je kritische kijk, motivatie en vertrouwen.

Peeter, elke dag stond je voor me klaar de afgelopen 5 jaar. Van 12 seconde meetings tot intellectuele discussies, maar ook 'trein naar Utrecht'-besprekingen en 'aai-overmijn-bol \& vrolijk-verder-onderzoeken' aanmoedigingen. Het verwonderde me elke keer weer dat ik zonder enige inleiding of uitleg onderzoeksvragen op je af kon vuren en dat jij binnen enkele seconden met een behulpzaam antwoord op de proppen kon komen. Ik heb veel geleerd van jouw absurd grote vakkennis en inspirerende en ambitieuze plannen. Ik ben je vooral dankbaar voor je onuitputtende zorg en geruststelling, zonder jou had dit boek hier nu niet gelegen. Ik, als $\mathrm{PhD}$ kindje, weet zeker dat jij een geweldige papa zult zijn. 


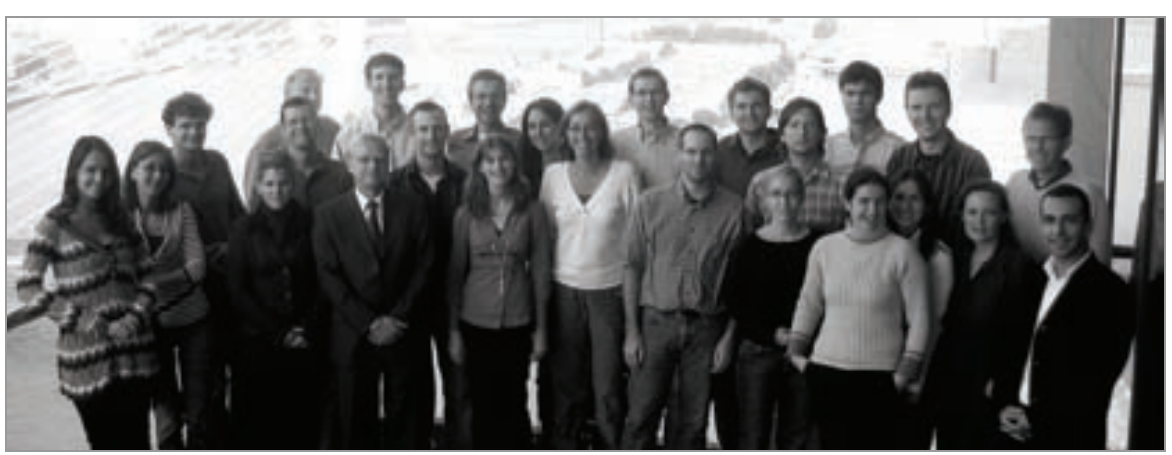

Daarnaast grote dank aan de afdeling Marketing Management voor een onvergetelijke tijd. Wat een krachtige afdeling! Hoewel het hoge niveau van onderzoek en ambitie me soms ontstelde heeft het me ook uitgedaagd tot mooie prestaties. Bedankt voor jullie kritische kijk, interesse, steun en gezelligheid. Met name Dirk, Stefano, Stijn en Bram erg bedankt voor jullie input op het paper in JCR en mijn andere projecten. Daarnaast wil ik Ralf, Berk, Jolanda, Steven, Maarten, Stefanie, Ezgi en Ilona bedanken voor hun hulp bij het creëren stimulus materiaal, draaien van studies of analyseren van data. Ook de PhD's en Postdoc van onze afdeling wil ik bedanken voor hun gezelligheid en onderzoekstips. We hebben wat afgelachen tijdens 'snelle' lunches en PhD-dinners. En waar zou onze afdeling zijn zonder zijn geweldige secretaresses: Annette en Jolanda? Bij jullie staat de deur altijd wagenwijd open, bedankt!

In het bijzonder wil ik vijf werkgerelateerde vriendinnetjes bedanken die mijn tijd in Rotterdam nog leuker hebben gemaakt.

Stefanie, de beste kamergenoot die iemand zich kan wensen. Onze tripjes naar de $4^{\text {de }}$ verdieping voor DE koffie waren een boost voor mijn creativiteit. Je bent de definitie van een allround-talent en ik ben trots dat ik op 4 november naast jou mag staan als jouw paranimf. Ilona, wat was het fijn om jou als collega te hebben. Ik heb erg genoten van je enthousiasme en zachtaardigheid. Ook wanneer het tegen zat kreeg jij me altijd weer op de been met een luiste-

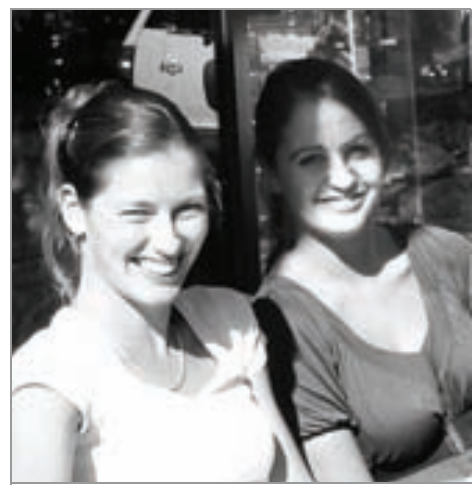
rend oor en praktische oplossingen, maar ook met een ontladend potje squash. Ezgi, I loved laughing with you. Thank you for everything and good luck with your thesis. 
Linde-Marie, ik ben blij dat ons sportcontact uitgegroeid is tot vele emails, lunches, etentjes en nog meer samen sporten. Bedankt voor je interesse, bezorgdheid en optimisme. Helleke, 's ochtends beginnen met thee op jouw kamer op onze verdieping, dat was ons ding. Allerlei werkgerelateerde en ongerelateerde zaken werden besproken en ik vond het erg jammer dat je de universiteit in ons tweede PhD-jaar verliet. Gelukkig hebben we er een mooie vriendschap voor teruggekregen!

Naast mijn leven als PhD in Rotterdam heb ik ook nog een leven in Nijmegen. Hierin omringen mij veel prachtige mensen die niets bij hebben gedragen aan mijn dissertatie, maar wel voor erg veel plezier en geluk zorgen! Jullie luisterden braaf als ik over mijn wetenschappelijke wereldje met zijn eigen spelregels vertelde, als ik droomde over mooie publicaties of juist stoom moest afblazen over frustraties. Bedankt dat jullie in mijn leven zijn! Hierbij wil ik de volgende mensen in het speciaal noemen:

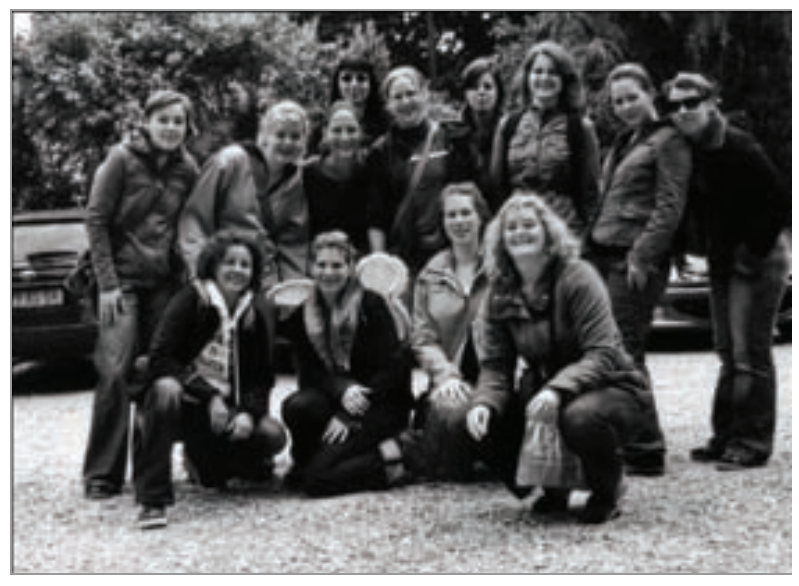

Mijn lieve vriendinnetjes, Lisette, Maaike, Kim, Rosalie, Ilse, Ellis, Yvette, Mijke, Brechje en Willemien. Mijn steun en toeverlaat! Met ieder van jullie heb ik een unieke band. Bedankt voor de afgelopen 5 jaar (en bij de meesten ook voor de velen jaren daarvoor) lekker kletsen en thee/koffie/wijn-leuten!

Mogen er nog vele jaren volgen. Ik ben dol op jullie!

Mijn paranimf Ilse in het speciaal (zie foto op p. 33). Uren heb je mijn gebabbel over Rotterdam, marketing, congressen en onderzoek aan willen horen. Bedankt voor het vragen om uitleg over dingen als experimenten en taalabstractie. En nee, uitleg vragen is niet dom, vragen is heerlijk, dat liet mij zien dat je mij echt wilde begrijpen en dat vond ik fijn. Het voelt goed om weten dat jij altijd achter me staat, wat ik ook doe, denk en voel. 
Mijn lieve familie en schoonfamilie als laatste. Mijn grote broer Rogier, bedankt voor de gezellige klets en steun. Els, Ernie en Tanja, sinds de eerste week van mijn promotietraject in mijn leven, bedankt voor het open ontvangst in jullie gezin en jullie gezelligheid.

Pap en mam, doordat jullie me hebben uitgedaagd te streven naar mijn dromen, heb ik dit boek kunnen schrijven. Jullie hebben mij de mogelijkheid en de vrijheid gegeven een raar pad te bewandelen via veel opleidingen in verschillende disciplines in Schijndel, Amerika, Eindhoven, Nijmegen en Rotterdam. Bedankt voor mijn thuis in Sint Michielsgestel, het heerlijke ontvangst van Yara en gewoon voor wie jullie zijn!

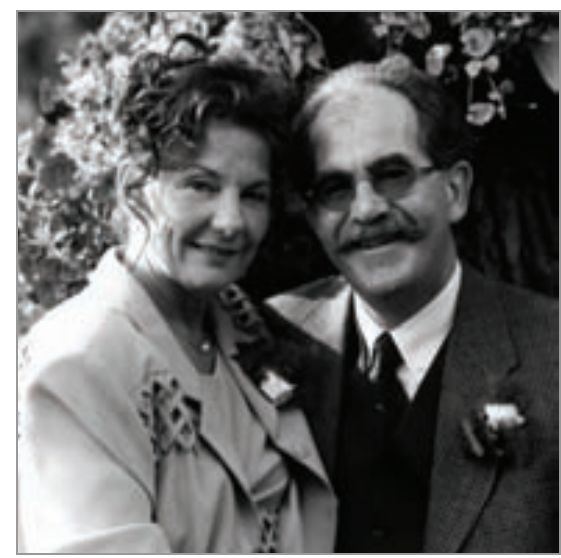

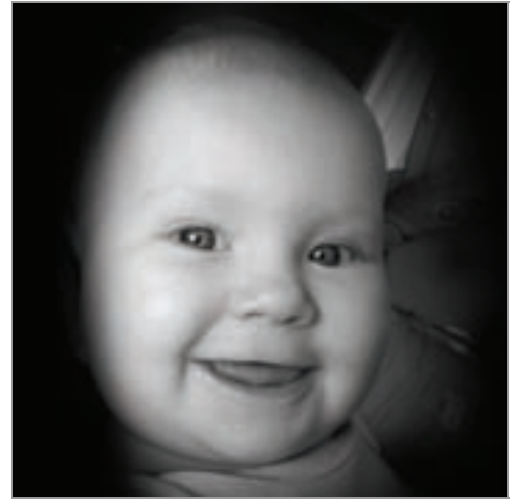

Tot slot, mijn lief Sjoerd, vier jaar lang accepteerde je het dat ik doordeweeks niet bij jou woonde. Het laatste half jaar reisde jij en Yara zelfs met me mee naar het westen. Bedankt voor het creëren van materiaal voor mijn experimenten en de voorkant van dit boek. Maar ik ben je vooral heel dankbaar voor je rust en liefde. Ik ben elke dag weer blij dat ik bij jou mag zijn. Je bent 't mooiste mens dat ik ken.

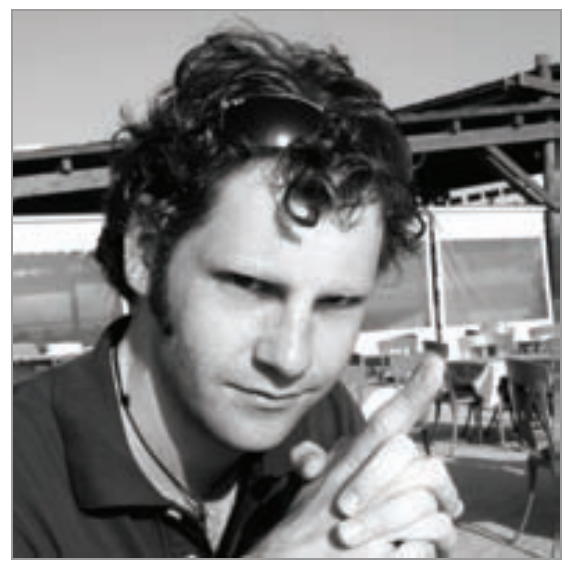




\section{About the author}

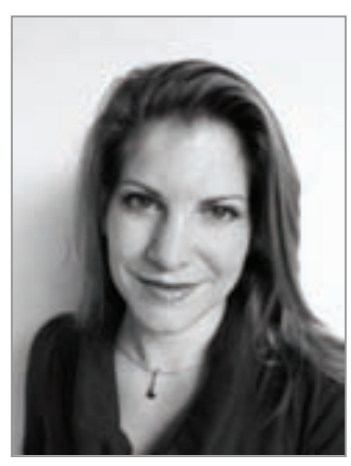

Gaby Anna Catharina Schellekens was born in 'sHertogenbosch, The Netherlands on September $11^{\text {th }}, 1978$. In 2000, she obtained a bachelors degree in Communication (HEAO Eindhoven) and then studied psychology at the Radboud University Nijmegen. She obtained a masters degree in Fundamental Social Psychology in 2005. Her thesis was on unconscious persuasion in consumer behavior and she completed this study with an internship at Unilever Research \& Development. In September 2005, Gaby became a $\mathrm{PhD}$ at the department Marketing Management at the Rotterdam School of Management, Erasmus University. Her PhD research is focused on language use in word of mouth. She presented her work at international conferences, such as Association for Consumer Research and the European Marketing Academy Conference. Gaby also published a mayor part of this dissertation in the Journal of Consumer Research (August 2010), the leading journal in consumer behavior. 



\title{
ERIM PhD Series
}

\author{
ERASMUS RESEARCH INSTITUTE OF MANAGEMENT (ERIM)
}

ERIM PH.D. SERIES

RESEARCH IN MANAGEMENT

ERIM Electronic Series Portal: http://hdl.handle.net/1765/1

Acciaro, M., Bundling Strategies in Global Supply Chains, Promotor: Prof.dr. H.E.

Haralambides, EPS-2010-197-LIS, ISBN: 978-90-5892-240-3, http://hdl.handle.net/1765/1

Althuizen, N.A.P., Analogical Reasoning as a Decision Support Principle for Weakly Structured Marketing Problems, Promotor: Prof.dr.ir. B. Wierenga, EPS-2006-095-MKT, ISBN: 90-5892129-8, http:/ /hdl.handle.net/1765/8190

Alvarez, H.L., Distributed Collaborative Learning Communities Enabled by Information Communication Technology, Promotor: Prof.dr. K. Kumar, EPS-2006-080-LIS, ISBN: 90-5892112-3, http:/ /hdl.handle.net/1765/7830

Appelman, J.H., Governance of Global Interorganizational Tourism Networks: Changing Forms of Co-ordination between the Travel Agency and Aviation Sector, Promotors: Prof.dr. F.M. Go \& Prof.dr. B. Nooteboom, EPS-2004-036-MKT, ISBN: 90-5892-060-7, http://hdl.handle.net/1765/1199

Asperen, E. van, Essays on Port, Container, and Bulk Chemical Logistics Optimization, Promotor: Prof.dr.ir. R. Dekker, EPS-2009-181-LIS, ISBN: 978-90-5892-222-9, http://hdl.handle.net/1765/1

Assem, M.J. van den, Deal or No Deal? Decision Making under Risk in a Large-Stake TV Game Show and Related Experiments, Promotor: Prof.dr. J. Spronk, EPS-2008-138-F\&A, ISBN: 97890-5892-173-4, http://hdl.handle.net/1765/13566 
Baquero, G, On Hedge Fund Performance, Capital Flows and Investor Psychology, Promotor: Prof.dr. M.J.C.M. Verbeek, EPS-2006-094-F\&A, ISBN: 90-5892-131-X, http://hdl.handle.net/1765/8192

Berens, G., Corporate Branding: The Development of Corporate Associations and their Influence on Stakeholder Reactions, Promotor: Prof.dr. C.B.M. van Riel, EPS-2004-039-ORG, ISBN: 905892-065-8, http://hdl.handle.net/1765/1273

Berghe, D.A.F. van den, Working Across Borders: Multinational Enterprises and the Internationalization of Employment, Promotors: Prof.dr. R.J.M. van Tulder \& Prof.dr. E.J.J. Schenk, EPS-2003-029-ORG, ISBN: 90-5892-05-34, http://hdl.handle.net/1765/1041

Berghman, L.A., Strategic Innovation Capacity: A Mixed Method Study on Deliberate Strategic Learning Mechanisms, Promotor: Prof.dr. P. Mattyssens, EPS-2006-087-MKT, ISBN: 905892-120-4, http://hdl.handle.net/1765/7991

Bezemer, P.J., Diffusion of Corporate Governance Beliefs: Board Independence and the Emergence of a Shareholder Value Orientation in the Netherlands, Promotors: Prof.dr.ing. F.A.J. van den Bosch \& Prof.dr. H.W. Volberda, EPS-2009-192-STR, ISBN: 978-90-5892-232-8, http://hdl.handle.net/1765/1

Bijman, W.J.J., Essays on Agricultural Co-operatives: Governance Structure in Fruit and Vegetable Chains, Promotor: Prof.dr. G.W.J. Hendrikse, EPS-2002-015-ORG, ISBN: 90-5892024-0, http://hdl.handle.net/1765/867

Bispo, A., Labour Market Segmentation: An investigation into the Dutch hospitality industry, Promotors: Prof.dr. G.H.M. Evers \& Prof.dr. A.R. Thurik, EPS-2007-108-ORG, ISBN: 905892-136-9, http://hdl.handle.net/1765/10283

Blindenbach-Driessen, F., Innovation Management in Project-Based Firms, Promotor: Prof.dr. S.L. van de Velde, EPS-2006-082-LIS, ISBN: 90-5892-110-7, http://hdl.handle.net/1765/7828

Boer, C.A., Distributed Simulation in Industry, Promotors: Prof.dr. A. de Bruin \& Prof.dr.ir. A. Verbraeck, EPS-2005-065-LIS, ISBN: 90-5892-093-3, http://hdl.handle.net/1765/6925 
Boer, N.I., Knowledge Sharing within Organizations: A situated and Relational Perspective, Promotor: Prof.dr. K. Kumar, EPS-2005-060-LIS, ISBN: 90-5892-086-0, http://hdl.handle.net/1765/6770

Boer-Sorbán, K., Agent-Based Simulation of Financial Markets: A modular, Continuous-Time Approach, Promotor: Prof.dr. A. de Bruin, EPS-2008-119-LIS, ISBN: 90-5892-155-0, http:/ /hdl.handle.net/1765/10870

Boon, C.T., HRM and Fit: Survival of the Fittest!?, Promotors: Prof.dr. J. Paauwe \& Prof.dr. D.N. den Hartog, EPS-2008-129-ORG, ISBN: 978-90-5892-162-8, http://hdl.handle.net/1765/12606

Braun, E., City Marketing: Towards an Integrated Approach, Promotor: Prof.dr. L. van den Berg, EPS-2008-142-MKT, ISBN: 978-90-5892-180-2, http://hdl.handle.net/1765/13694

Brito, M.P. de, Managing Reverse Logistics or Reversing Logistics Management? Promotors: Prof.dr.ir. R. Dekker \& Prof.dr. M. B. M. de Koster, EPS-2004-035-LIS, ISBN: 90-5892-0585, http://hdl.handle.net/1765/1132

Brohm, R., Polycentric Order in Organizations: A Dialogue between Michael Polanyi and ITConsultants on Knowledge, Morality, and Organization, Promotors: Prof.dr. G. W. J. Hendrikse \& Prof.dr. H. K. Letiche, EPS-2005-063-ORG, ISBN: 90-5892-095-X, http://hdl.handle.net/1765/6911

Brumme, W.-H., Manufacturing Capability Switching in the High-Tech Electronics Technology Life Cycle, Promotors: Prof.dr.ir. J.A.E.E. van Nunen \& Prof.dr.ir. L.N. Van Wassenhove, EPS-2008-126-LIS, ISBN: 978-90-5892-150-5, http://hdl.handle.net/1765/12103

Budiono, D.P., The Analysis of Mutual Fund Performance: Evidence from U.S. Equity Mutual Funds, Promotor: Prof.dr.ir. M.J.C.M. Verbeek, EPS-2010-185-F\&A, ISBN: 978-905892-224-3, http://hdl.handle.net/1765/1

Burgers, J.H., Managing Corporate Venturing: Multilevel Studies on Project Autonomy, Integration, Knowledge Relatedness, and Phases in the New Business Development Process, Promotors: Prof.dr.ir. F.A.J. Van den Bosch \& Prof.dr. H.W. Volberda, EPS-2008-136-STR, ISBN: 978-90-5892-174-1, http://hdl.handle.net/1765/13484 
Campbell, R.A.J., Rethinking Risk in International Financial Markets, Promotor: Prof.dr. C.G. Koedijk, EPS-2001-005-F\&A, ISBN: 90-5892-008-9, http://hdl.handle.net/1765/306

Carvalho de Mesquita Ferreira, L., Attention Mosaics: Studies of Organizational Attention, Promotors: Prof.dr. P.M.A.R. Heugens \& Prof.dr. J. van Oosterhout, EPS-2010-205-ORG, ISBN: 978-90-5892-242-7, http://hdl.handle.net/1765/1

Chen, C.-M., Evaluation and Design of Supply Chain Operations Using DEA, Promotor: Prof.dr. J.A.E.E. van Nunen, EPS-2009-172-LIS, ISBN: 978-90-5892-209-0, http://hdl.handle.net/1765/1

Chen, H., Individual Mobile Communication Services and Tariffs, Promotor: Prof.dr. L.F.J.M. Pau, EPS-2008-123-LIS, ISBN: 90-5892-158-1, http://hdl.handle.net/1765/11141

Chen, Y., Labour Flexibility in China's Companies: An Empirical Study, Promotors: Prof.dr. A. Buitendam \& Prof.dr. B. Krug, EPS-2001-006-ORG, ISBN: 90-5892-012-7, http:/ /hdl.handle.net/1765/307

Damen, F.J.A., Taking the Lead: The Role of Affect in Leadership Effectiveness, Promotor:

Prof.dr. D.L. van Knippenberg, EPS-2007-107-ORG, http://hdl.handle.net/1765/10282

Daniševská, P., Empirical Studies on Financial Intermediation and Corporate Policies, Promotor: Prof.dr. C.G. Koedijk, EPS-2004-044-F\&A, ISBN: 90-5892-070-4, http://hdl.handle.net/1765/1518

Defilippi Angeldonis, E.F., Access Regulation for Naturally Monopolistic Port Terminals: Lessons from Regulated Network Industries, Promotor: Prof.dr. H.E. Haralambides, EPS2010-204-LIS, ISBN:

978-90-5892-245-8, http://hdl.handle.net/1765/1

Delporte-Vermeiren, D.J.E., Improving the Flexibility and Profitability of ICT-enabled Business Networks: An Assessment Method and Tool, Promotors: Prof. mr. dr. P.H.M. Vervest \& Prof.dr.ir. H.W.G.M. van Heck, EPS-2003-020-LIS, ISBN: 90-5892-040-2, http://hdl.handle.net/1765/359

Derwall, J.M.M., The Economic Virtues of SRI and CSR, Promotor: Prof.dr. C.G. Koedijk, EPS-2007-101-F\&A, ISBN: 90-5892-132-8, http://hdl.handle.net/1765/8986 
Diepen, M. van, Dynamics and Competition in Charitable Giving, Promotor: Prof.dr. Ph.H.B.F. Franses, EPS-2009-159-MKT, ISBN: 978-90-5892-188-8, http://hdl.handle.net/1765/14526

Dietz, H.M.S., Managing (Sales)People towards Perfomance: HR Strategy, Leadership $\mathcal{E}$ Teamwork, Promotor: Prof.dr. G.W.J. Hendrikse, EPS-2009-168-ORG, ISBN: 978-90-5892210-6, http://hdl.handle.net/1765/1

Dijksterhuis, M., Organizational Dynamics of Cognition and Action in the Changing Dutch and US Banking Industries, Promotors: Prof.dr.ir. F.A.J. van den Bosch \& Prof.dr. H.W. Volberda, EPS-2003-026-STR, ISBN: 90-5892-048-8, http://hdl.handle.net/1765/1037

Eijk, A.R. van der, Behind Networks: Knowledge Transfer, Favor Exchange and Performance, Promotors: Prof.dr. S.L. van de Velde \& Prof.dr.drs. W.A. Dolfsma, EPS-2009-161-LIS, ISBN: 978-90-5892-190-1, http://hdl.handle.net/1765/14613

Elstak, M.N., Flipping the Identity Coin: The Comparative Effect of Perceived, Projected and Desired Organizational Identity on Organizational Identification and Desired Behavior, Promotor: Prof.dr. C.B.M. van Riel, EPS-2008-117-ORG, ISBN: 90-5892-148-2, http://hdl.handle.net/1765/10723

Erken, H.P.G., Productivity, RED and Entrepreneurship, Promotor: Prof.dr. A.R. Thurik, EPS-2008-147-ORG, ISBN: 978-90-5892-179-6, http:/ /hdl.handle.net/1765/14004

Fenema, P.C. van, Coordination and Control of Globally Distributed Software Projects, Promotor: Prof.dr. K. Kumar, EPS-2002-019-LIS, ISBN: 90-5892-030-5, http:/ / hdl.handle.net/1765/360

Fleischmann, M., Quantitative Models for Reverse Logistics, Promotors: Prof.dr.ir. J.A.E.E. van Nunen \& Prof.dr.ir. R. Dekker, EPS-2000-002-LIS, ISBN: 35-4041-711-7, http://hdl.handle.net/1765/1044

Flier, B., Strategic Renewal of European Financial Incumbents: Coevolution of Environmental Selection, Institutional Effects, and Managerial Intentionality, Promotors: Prof.dr.ir. F.A.J. van den Bosch \& Prof.dr. H.W. Volberda, EPS-2003-033-STR, ISBN: 90-5892-055-0, http://hdl.handle.net/1765/1071 
Fok, D., Advanced Econometric Marketing Models, Promotor: Prof.dr. Ph.H.B.F. Franses, EPS-2003-027-MKT, ISBN: 90-5892-049-6, http://hdl.handle.net/1765/1035

Ganzaroli, A., Creating Trust between Local and Global Systems, Promotors: Prof.dr. K. Kumar \& Prof.dr. R.M. Lee, EPS-2002-018-LIS, ISBN: 90-5892-031-3, http://hdl.handle.net/1765/361

Gertsen, H.F.M., Riding a Tiger without Being Eaten: How Companies and Analysts Tame Financial Restatements and Influence Corporate Reputation, Promotor: Prof.dr. C.B.M. van Riel, EPS-2009-171-ORG, ISBN: 90-5892-214-4, http://hdl.handle.net/1765/1

Gilsing, V.A., Exploration, Exploitation and Co-evolution in Innovation Networks, Promotors: Prof.dr. B. Nooteboom \& Prof.dr. J.P.M. Groenewegen, EPS-2003-032-ORG, ISBN: 905892-054-2, http://hdl.handle.net/1765/1040

Gijsbers, G.W., Agricultural Innovation in Asia: Drivers, Paradigms and Performance, Promotor: Prof.dr. R.J.M. van Tulder, EPS-2009-156-ORG, ISBN: 978-90-5892-191-8, http://hdl.handle.net/1765/14524

Gong, Y., Stochastic Modelling and Analysis of Warehouse Operations, Promotors: Prof.dr. M.B.M. de Koster \& Prof.dr. S.L. van de Velde, EPS-2009-180-LIS, ISBN: 978-90-5892-2199, http://hdl.handle.net/1765/1

Govers, R., Virtual Tourism Destination Image: Glocal Identities Constructed, Perceived and Experienced, Promotors: Prof.dr. F.M. Go \& Prof.dr. K. Kumar, EPS-2005-069-MKT, ISBN: 90-5892-107-7, http://hdl.handle.net/1765/6981

Graaf, G. de, Tractable Morality: Customer Discourses of Bankers, Veterinarians and Charity Workers, Promotors: Prof.dr. F. Leijnse \& Prof.dr. T. van Willigenburg, EPS-2003-031ORG, ISBN: 90-5892-051-8, http://hdl.handle.net/1765/1038

Greeven, M.J., Innovation in an Uncertain Institutional Environment: Private Software Entrepreneurs in Hangzhou, China, Promotor: Prof.dr. B. Krug, EPS-2009-164-ORG, ISBN: 978-90-5892-202-1, http://hdl.handle.net/1765/15426

Groot, E.A. de, Essays on Economic Cycles, Promotors: Prof.dr. Ph.H.B.F. Franses \& Prof.dr. H.R. Commandeur, EPS-2006-091-MKT, ISBN: 90-5892-123-9, http://hdl.handle.net/1765/8216 
Guenster, N.K., Investment Strategies Based on Social Responsibility and Bubbles, Promotor: Prof.dr. C.G. Koedijk, EPS-2008-175-F\&A, ISBN: 978-90-5892-206-9, http://hdl.handle.net/1765/1

Gutkowska, A.B., Essays on the Dynamic Portfolio Choice, Promotor: Prof.dr. A.C.F. Vorst, EPS-2006-085-F\&A, ISBN: 90-5892-118-2, http://hdl.handle.net/1765/7994

Hagemeijer, R.E., The Unmasking of the Other, Promotors: Prof.dr. S.J. Magala \& Prof.dr. H.K. Letiche, EPS-2005-068-ORG, ISBN: 90-5892-097-6, http://hdl.handle.net/1765/6963

Hakimi, N.A, Leader Empowering Behaviour: The Leader's Perspective: Understanding the Motivation behind Leader Empowering Behaviour, Promotor: Prof.dr. D.L. van Knippenberg, EPS-2010-184-ORG, http://hdl.handle.net/1765/1

Halderen, M.D. van, Organizational Identity Expressiveness and Perception Management: Principles for Expressing the Organizational Identity in Order to Manage the Perceptions and Behavioral Reactions of External Stakeholders, Promotor: Prof.dr. S.B.M. van Riel, EPS-2008122-ORG, ISBN: 90-5892-153-6, http://hdl.handle.net/1765/10872

Hartigh, E. den, Increasing Returns and Firm Performance: An Empirical Study, Promotor: Prof.dr. H.R. Commandeur, EPS-2005-067-STR, ISBN: 90-5892-098-4, http:/ /hdl.handle.net/1765/6939

Hensmans, M., A Republican Settlement Theory of the Firm: Applied to Retail Banks in England and the Netherlands (1830-2007), Promotors: Prof.dr. A. Jolink\& Prof.dr. S.J. Magala, EPS2010-193-ORG, ISBN 90-5892-235-9, http://hdl.handle.net/1765/1

Hermans. J.M., ICT in Information Services; Use and Deployment of the Dutch Securities Trade, 1860-1970, Promotor: Prof.dr. drs. F.H.A. Janszen, EPS-2004-046-ORG, ISBN 90-5892-0720, http://hdl.handle.net/1765/1793

Hernandez Mireles, C., Marketing Modeling for New Products, Promotor: Prof.dr. P.H. Franses, EPS-2010-202-MKT, ISBN 90-5892-237-3, http:/ /hdl.handle.net/1765/1

Hessels, S.J.A., International Entrepreneurship: Value Creation Across National Borders, Promotor: Prof.dr. A.R. Thurik, EPS-2008-144-ORG, ISBN: 978-90-5892-181-9, http://hdl.handle.net/1765/13942 
Heugens, P.P.M.A.R., Strategic Issues Management: Implications for Corporate Performance, Promotors: Prof.dr.ir. F.A.J. van den Bosch \& Prof.dr. C.B.M. van Riel, EPS-2001-007-STR, ISBN: 90-5892-009-9, http://hdl.handle.net/1765/358

Heuvel, W. van den, The Economic Lot-Sizing Problem: New Results and Extensions, Promotor: Prof.dr. A.P.L. Wagelmans, EPS-2006-093-LIS, ISBN: 90-5892-124-7, http://hdl.handle.net/1765/1805

Hoedemaekers, C.M.W., Performance, Pinned down: A Lacanian Analysis of Subjectivity at Work, Promotors: Prof.dr. S. Magala \& Prof.dr. D.H. den Hartog, EPS-2008-121-ORG, ISBN: 90-5892-156-7, http://hdl.handle.net/1765/10871

Hooghiemstra, R., The Construction of Reality: Cultural Differences in Self-serving Behaviour in Accounting Narratives, Promotors: Prof.dr. L.G. van der Tas RA \& Prof.dr. A.Th.H. Pruyn, EPS-2003-025-F\&A, ISBN: 90-5892-047-X, http://hdl.handle.net/1765/871

$\mathrm{Hu}$, Y., Essays on the Governance of Agricultural Products: Cooperatives and Contract Farming, Promotors: Prof.dr. G.W.J. Hendrkse \& Prof.dr. B. Krug, EPS-2007-113-ORG, ISBN: 905892-145-1, http://hdl.handle.net/1765/10535

Huang, X., An Analysis of Occupational Pension Provision: From Evaluation to Redesigh, Promotors: Prof.dr. M.J.C.M. Verbeek \& Prof.dr. R.J. Mahieu, EPS-2010-196-F\&A, ISBN: 90-5892-239-7, http://hdl.handle.net/1765/1

Huij, J.J., New Insights into Mutual Funds: Performance and Family Strategies, Promotor: Prof.dr. M.C.J.M. Verbeek, EPS-2007-099-F\&A, ISBN: 90-5892-134-4, http:/ /hdl.handle.net/1765/9398

Huurman, C.I., Dealing with Electricity Prices, Promotor: Prof.dr. C.D. Koedijk, EPS-2007098-F\&A, ISBN: 90-5892-130-1, http://hdl.handle.net/1765/9399

Iastrebova, K, Manager's Information Overload: The Impact of Coping Strategies on DecisionMaking Performance, Promotor: Prof.dr. H.G. van Dissel, EPS-2006-077-LIS, ISBN: 90-5892111-5, http:/ /hdl.handle.net/1765/7329

Iwaarden, J.D. van, Changing Quality Controls: The Effects of Increasing Product Variety and Shortening Product Life Cycles, Promotors: Prof.dr. B.G. Dale \& Prof.dr. A.R.T. Williams, EPS-2006-084-ORG, ISBN: 90-5892-117-4, http://hdl.handle.net/1765/7992 
Jansen, J.J.P., Ambidextrous Organizations, Promotors: Prof.dr.ir. F.A.J. Van den Bosch \& Prof.dr. H.W. Volberda, EPS-2005-055-STR, ISBN: 90-5892-081-X, http:/ /hdl.handle.net/1765/6774

Jaspers, F.P.H., Organizing Systemic Innovation, Promotor: Prof.dr.ir. J.C.M. van den Ende, EPS-2009-160-ORG, ISBN: 978-90-5892-197-), http:/ /hdl.handle.net/1765/14974

Jennen, M.G.J., Empirical Essays on Office Market Dynamics, Promotors: Prof.dr. C.G. Koedijk \& Prof.dr. D. Brounen, EPS-2008-140-F\&A, ISBN: 978-90-5892-176-5, http://hdl.handle.net/1765/13692

Jiang, T., Capital Structure Determinants and Governance Structure Variety in Franchising, Promotors: Prof.dr. G. Hendrikse \& Prof.dr. A. de Jong, EPS-2009-158-F\&A, ISBN: 978-905892-199-4, http://hdl.handle.net/1765/14975

Jiao, T., Essays in Financial Accounting, Promotor: Prof.dr. G.M.H. Mertens, EPS-2009-176F\&A, ISBN: 978-90-5892-211-3, http://hdl.handle.net/1765/1

Jong, C. de, Dealing with Derivatives: Studies on the Role, Informational Content and Pricing of Financial Derivatives, Promotor: Prof.dr. C.G. Koedijk, EPS-2003-023-F\&A, ISBN: 90-5892043-7, http://hdl.handle.net/1765/1043

Kaa, G. van, Standard Battles for Complex Systems: Empirical Research on the Home Network, Promotors: Prof.dr.ir. J. van den Ende \& Prof.dr.ir. H.W.G.M. van Heck, EPS-2009-166ORG, ISBN: 978-90-5892-205-2, http://hdl.handle.net/1765/1

Kagie, M., Advances in Online Shopping Interfaces: Product Catalog Maps and Recommender Systems, Promotor: Prof.dr. P.J.F. Groenen, EPS-2010-195-MKT, ISBN: 978-90-5892-233-5, http://hdl.handle.net/1765/1

Keizer, A.B., The Changing Logic of Japanese Employment Practices: A Firm-Level Analysis of Four Industries, Promotors: Prof.dr. J.A. Stam \& Prof.dr. J.P.M. Groenewegen, EPS-2005057-ORG, ISBN: 90-5892-087-9, http://hdl.handle.net/1765/6667

Kijkuit, R.C., Social Networks in the Front End: The Organizational Life of an Idea, Promotor: Prof.dr. B. Nooteboom, EPS-2007-104-ORG, ISBN: 90-5892-137-6, http://hdl.handle.net/1765/10074 
Kippers, J., Empirical Studies on Cash Payments, Promotor: Prof.dr. Ph.H.B.F. Franses, EPS2004-043-F\&A, ISBN: 90-5892-069-0, http://hdl.handle.net/1765/1520

Klein, M.H., Poverty Alleviation through Sustainable Strategic Business Models: Essays on Poverty Alleviation as a Business Strategy, Promotor: Prof.dr. H.R. Commandeur, EPS-2008135-STR, ISBN: 978-90-5892-168-0, http://hdl.handle.net/1765/13482

Knapp, S., The Econometrics of Maritime Safety: Recommendations to Enhance Safety at Sea, Promotor: Prof.dr. Ph.H.B.F. Franses, EPS-2007-096-ORG, ISBN: 90-5892-127-1, http://hdl.handle.net/1765/7913

Kole, E., On Crises, Crashes and Comovements, Promotors: Prof.dr. C.G. Koedijk \& Prof.dr. M.J.C.M. Verbeek, EPS-2006-083-F\&A, ISBN: 90-5892-114-X, http://hdl.handle.net/1765/7829

Kooij-de Bode, J.M., Distributed Information and Group Decision-Making: Effects of Diversity and Affect, Promotor: Prof.dr. D.L. van Knippenberg, EPS-2007-115-ORG, http://hdl.handle.net/1765/10722

Koppius, O.R., Information Architecture and Electronic Market Performance, Promotors: Prof.dr. P.H.M. Vervest \& Prof.dr.ir. H.W.G.M. van Heck, EPS-2002-013-LIS, ISBN: 905892-023-2, http://hdl.handle.net/1765/921

Kotlarsky, J., Management of Globally Distributed Component-Based Software Development Projects, Promotor: Prof.dr. K. Kumar, EPS-2005-059-LIS, ISBN: 90-5892-088-7, http://hdl.handle.net/1765/6772

Krauth, E.I., Real-Time Planning Support: A Task-Technology Fit Perspective, Promotors: Prof.dr. S.L. van de Velde \& Prof.dr. J. van Hillegersberg, EPS-2008-155-LIS, ISBN: 978-905892-193-2, http://hdl.handle.net/1765/14521

Kuilman, J., The Re-Emergence of Foreign Banks in Shanghai: An Ecological Analysis, Promotor: Prof.dr. B. Krug, EPS-2005-066-ORG, ISBN: 90-5892-096-8, http://hdl.handle.net/1765/6926

Kwee, Z., Investigating Three Key Principles of Sustained Strategic Renewal: A Longitudinal Study of Long-Lived Firms, Promotors: Prof.dr.ir. F.A.J. Van den Bosch \& Prof.dr. H.W. Volberda, EPS-2009-174-STR, ISBN: 90-5892-212-0, http://hdl.handle.net/1765/1 
Langen, P.W. de, The Performance of Seaport Clusters: A Framework to Analyze Cluster Performance and an Application to the Seaport Clusters of Durban, Rotterdam and the Lower Mississippi, Promotors: Prof.dr. B. Nooteboom \& Prof. drs. H.W.H. Welters, EPS-2004-034LIS, ISBN: 90-5892-056-9, http://hdl.handle.net/1765/1133

Le Anh, T., Intelligent Control of Vehicle-Based Internal Transport Systems, Promotors: Prof.dr. M.B.M. de Koster \& Prof.dr.ir. R. Dekker, EPS-2005-051-LIS, ISBN: 90-5892-079-8, http://hdl.handle.net/1765/6554

Le-Duc, T., Design and Control of Efficient Order Picking Processes, Promotor: Prof.dr. M.B.M. de Koster, EPS-2005-064-LIS, ISBN: 90-5892-094-1, http://hdl.handle.net/1765/6910

Leeuwen, E.P. van, Recovered-Resource Dependent Industries and the Strategic Renewal of Incumbent Firm: A Multi-Level Study of Recovered Resource Dependence Management and Strategic Renewal in the European Paper and Board Industry, Promotors: Prof.dr.ir. F.A.J. Van den Bosch \& Prof.dr. H.W. Volberda, EPS-2007-109-STR, ISBN: 90-5892-140-6, http://hdl.handle.net/1765/10183

Lentink, R.M., Algorithmic Decision Support for Shunt Planning, Promotors: Prof.dr. L.G. Kroon \& Prof.dr.ir. J.A.E.E. van Nunen, EPS-2006-073-LIS, ISBN: 90-5892-104-2, http:/ /hdl.handle.net/1765/7328

Li, T., Informedness and Customer-Centric Revenue Management, Promotors: Prof.dr. P.H.M. Vervest \& Prof.dr.ir. H.W.G.M. van Heck, EPS-2009-146-LIS, ISBN: 978-90-5892-195-6, http://hdl.handle.net/1765/14525

Liang, G., New Competition: Foreign Direct Investment and Industrial Development in China, Promotor: Prof.dr. R.J.M. van Tulder, EPS-2004-047-ORG, ISBN: 90-5892-073-9, http://hdl.handle.net/1765/1795

Liere, D.W. van, Network Horizon and the Dynamics of Network Positions: A Multi-Method Multi-Level Longitudinal Study of Interfirm Networks, Promotor: Prof.dr. P.H.M. Vervest, EPS-2007-105-LIS, ISBN: 90-5892-139-0, http://hdl.handle.net/1765/10181

Loef, J., Incongruity between Ads and Consumer Expectations of Advertising, Promotors: Prof.dr. W.F. van Raaij \& Prof.dr. G. Antonides, EPS-2002-017-MKT, ISBN: 90-5892-028-3, http:/ /hdl.handle.net/1765/869 
Maeseneire, W., de, Essays on Firm Valuation and Value Appropriation, Promotor: Prof.dr. J.T.J. Smit, EPS-2005-053-F\&A, ISBN: 90-5892-082-8, http://hdl.handle.net/1765/6768

Londoño, M. del Pilar, Institutional Arrangements that Affect Free Trade Agreements: Economic Rationality Versus Interest Groups, Promotors: Prof.dr. H.E. Haralambides \& Prof.dr. J.F. Francois, EPS-2006-078-LIS, ISBN: 90-5892-108-5, http://hdl.handle.net/1765/7578

Maas, A.A., van der, Strategy Implementation in a Small Island Context: An Integrative Framework, Promotor: Prof.dr. H.G. van Dissel, EPS-2008-127-LIS, ISBN: 978-90-5892-1604, http:/ /hdl.handle.net/1765/12278

Maas, K.E.G., Corporate Social Performance: From Output Measurement to Impact Measurement, Promotor: Prof.dr. H.R. Commandeur, EPS-2009-182-STR, ISBN: 978-905892-225-0, http:/ /hdl.handle.net/1765/1

Maeseneire, W., de, Essays on Firm Valuation and Value Appropriation, Promotor: Prof.dr. J.T.J. Smit, EPS-2005-053-F\&A, ISBN: 90-5892-082-8, http://hdl.handle.net/1765/6768

Mandele, L.M., van der, Leadership and the Inflection Point: A Longitudinal Perspective, Promotors: Prof.dr. H.W. Volberda \& Prof.dr. H.R. Commandeur, EPS-2004-042-STR, ISBN: 90-5892-067-4, http:/ /hdl.handle.net/1765/1302

Meer, J.R. van der, Operational Control of Internal Transport, Promotors: Prof.dr. M.B.M. de Koster \& Prof.dr.ir. R. Dekker, EPS-2000-001-LIS, ISBN: 90-5892-004-6, http://hdl.handle.net/1765/859

Mentink, A., Essays on Corporate Bonds, Promotor: Prof.dr. A.C.F. Vorst, EPS-2005-070F\&A, ISBN: 90-5892-100-X, http://hdl.handle.net/1765/7121

Meyer, R.J.H., Mapping the Mind of the Strategist: A Quantitative Methodology for Measuring the Strategic Beliefs of Executives, Promotor: Prof.dr. R.J.M. van Tulder, EPS-2007-106-ORG, ISBN: 978-90-5892-141-3, http://hdl.handle.net/1765/10182

Miltenburg, P.R., Effects of Modular Sourcing on Manufacturing Flexibility in the Automotive Industry: A Study among German OEMs, Promotors: Prof.dr. J. Paauwe \& Prof.dr. H.R. Commandeur, EPS-2003-030-ORG, ISBN: 90-5892-052-6, http://hdl.handle.net/1765/1039 
Moerman, G.A., Empirical Studies on Asset Pricing and Banking in the Euro Area, Promotor:

Prof.dr. C.G. Koedijk, EPS-2005-058-F\&A, ISBN: 90-5892-090-9,

http://hdl.handle.net/1765/6666

Moitra, D., Globalization of RED: Leveraging Offshoring for Innovative Capability and Organizational Flexibility, Promotor: Prof.dr. K. Kumar, EPS-2008-150-LIS, ISBN: 978-905892-184-0, http://hdl.handle.net/1765/14081

Mol, M.M., Outsourcing, Supplier-relations and Internationalisation: Global Source Strategy as a Chinese Puzzle, Promotor: Prof.dr. R.J.M. van Tulder, EPS-2001-010-ORG, ISBN: 90-5892014-3, http:/ / hdl.handle.net/1765/355

Mom, T.J.M., Managers' Exploration and Exploitation Activities: The Influence of Organizational Factors and Knowledge Inflows, Promotors: Prof.dr.ir. F.A.J. Van den Bosch \& Prof.dr. H.W. Volberda, EPS-2006-079-STR, ISBN: 90-5892-116-6, http:/ /hdl.handle.net/1765

Moonen, J.M., Multi-Agent Systems for Transportation Planning and Coordination, Promotors: Prof.dr. J. van Hillegersberg \& Prof.dr. S.L. van de Velde, EPS-2009-177-LIS, ISBN: 978-905892-216-8, http://hdl.handle.net/1

Mulder, A., Government Dilemmas in the Private Provision of Public Goods, Promotor: Prof.dr. R.J.M. van Tulder, EPS-2004-045-ORG, ISBN: 90-5892-071-2, http://hdl.handle.net/1765/1790

Muller, A.R., The Rise of Regionalism: Core Company Strategies Under The Second Wave of Integration, Promotor: Prof.dr. R.J.M. van Tulder, EPS-2004-038-ORG, ISBN: 90-5892-062-3, http://hdl.handle.net/1765/1272

Nalbantov G.I., Essays on Some Recent Penalization Methods with Applications in Finance and Marketing, Promotor: Prof. dr P.J.F. Groenen, EPS-2008-132-F\&A, ISBN: 978-90-5892-166-6, http://hdl.handle.net/1765/13319

Nederveen Pieterse, A., Goal Orientation in Teams: The Role of Diversity, Promotor: Prof.dr. D.L. van Knippenberg, EPS-2009-162-ORG, http://hdl.handle.net/1765/15240

Nguyen, T.T., Capital Structure, Strategic Competition, and Governance, Promotor: Prof.dr. A. de Jong, EPS-2008-148-F\&A, ISBN: 90-5892-178-9, http://hdl.handle.net/1765/14005 
Niesten, E.M.M.I., Regulation, Governance and Adaptation: Governance Transformations in the Dutch and French Liberalizing Electricity Industries, Promotors: Prof.dr. A. Jolink \& Prof.dr. J.P.M. Groenewegen, EPS-2009-170-ORG, ISBN: 978-90-5892-208-3, http://hdl.handle.net/1765/1

Nieuwenboer, N.A. den, Seeing the Shadow of the Self, Promotor: Prof.dr. S.P. Kaptein, EPS2008-151-ORG, ISBN: 978-90-5892-182-6, http://hdl.handle.net/1765/14223

Ning, H., Hierarchical Portfolio Management: Theory and Applications, Promotor: Prof.dr. J. Spronk, EPS-2007-118-F\&A, ISBN: 90-5892-152-9, http://hdl.handle.net/1765/10868

Noeverman, J., Management Control Systems, Evaluative Style, and Behaviour: Exploring the Concept and Behavioural Consequences of Evaluative Style, Promotors: Prof.dr. E.G.J. Vosselman \& Prof.dr. A.R.T. Williams, EPS-2007-120-F\&A, ISBN: 90-5892-151-2, http://hdl.handle.net/1765/10869

Nuijten, I., Servant Leadership: Paradox or Diamond in the Rough? A Multidimensional Measure and Empirical Evidence, Promotor: Prof.dr. D.L. van Knippenberg, EPS-2009-183ORG, http://hdl.handle.net/1765/1

Oosterhout, J., van, The Quest for Legitimacy: On Authority and Responsibility in Governance, Promotors: Prof.dr. T. van Willigenburg \& Prof.mr. H.R. van Gunsteren, EPS-2002-012ORG, ISBN: 90-5892-022-4, http://hdl.handle.net/1765/362

Oosterhout, M., van, Business Agility and Information Technology in Service Organizations, Promotor: Prof,dr.ir. H.W.G.M. van Heck, EPS-2010-198-LIS, ISBN: 90-5092-236-6, http://hdl.handle.net/1765/1

Oostrum, J.M., van, Applying Mathematical Models to Surgical Patient Planning, Promotor: Prof.dr. A.P.M. Wagelmans, EPS-2009-179-LIS, ISBN: 978-90-5892-217-5, http://hdl.handle.net/1765/1

Paape, L., Corporate Governance: The Impact on the Role, Position, and Scope of Services of the Internal Audit Function, Promotors: Prof.dr. G.J. van der Pijl \& Prof.dr. H. Commandeur, EPS-2007-111-MKT, ISBN: 90-5892-143-7, http:/ /hdl.handle.net/1765/10417

Pak, K., Revenue Management: New Features and Models, Promotor: Prof.dr.ir. R. Dekker, EPS-2005-061-LIS, ISBN: 90-5892-092-5, http://hdl.handle.net/1765/362/6771 
Pattikawa, L.H, Innovation in the Pharmaceutical Industry: Evidence from Drug Introduction in the U.S., Promotors: Prof.dr. H.R.Commandeur, EPS-2007-102-MKT, ISBN: 90-5892-135-2, http:/ /hdl.handle.net/1765/9626

Peeters, L.W.P., Cyclic Railway Timetable Optimization, Promotors: Prof.dr. L.G. Kroon \& Prof.dr.ir. J.A.E.E. van Nunen, EPS-2003-022-LIS, ISBN: 90-5892-042-9, http:/ /hdl.handle.net/1765/429

Pietersz, R., Pricing Models for Bermudan-style Interest Rate Derivatives, Promotors: Prof.dr. A.A.J. Pelsser \& Prof.dr. A.C.F. Vorst, EPS-2005-071-F\&A, ISBN: 90-5892-099-2, http://hdl.handle.net/1765/7122

Pince, C., Advances in Inventory Management: Dynamic Models, Promotor: Prof.dr.ir. R. Dekker, EPS-2010-199-LIS, ISBN: 978-90-5892-243-4, http:/ /hdl.handle.net/1765/1

Poel, A.M. van der, Empirical Essays in Corporate Finance and Financial Reporting, Promotors: Prof.dr. A. de Jong \& Prof.dr. G.M.H. Mertens, EPS-2007-133-F\&A, ISBN: 97890-5892-165-9, http://hdl.handle.net/1765/13320

Popova, V., Knowledge Discovery and Monotonicity, Promotor: Prof.dr. A. de Bruin, EPS2004-037-LIS, ISBN: 90-5892-061-5, http://hdl.handle.net/1765/1201

Pouchkarev, I., Performance Evaluation of Constrained Portfolios, Promotors: Prof.dr. J. Spronk \& Dr. W.G.P.M. Hallerbach, EPS-2005-052-F\&A, ISBN: 90-5892-083-6, http://hdl.handle.net/1765/6731

Prins, R., Modeling Consumer Adoption and Usage of Value-Added Mobile Services, Promotors: Prof.dr. Ph.H.B.F. Franses \& Prof.dr. P.C. Verhoef, EPS-2008-128-MKT, ISBN: 978/905892-161-1, http://hdl.handle.net/1765/12461

Puvanasvari Ratnasingam, P., Interorganizational Trust in Business to Business E-Commerce, Promotors: Prof.dr. K. Kumar \& Prof.dr. H.G. van Dissel, EPS-2001-009-LIS, ISBN: 905892-017-8, http://hdl.handle.net/1765/356

Quak, H.J., Sustainability of Urban Freight Transport: Retail Distribution and Local Regulation in Cities, Promotor: Prof.dr. M.B.M. de Koster, EPS-2008-124-LIS, ISBN: 978-90-5892-154-3, http://hdl.handle.net/1765/11990 
Quariguasi Frota Neto, J., Eco-efficient Supply Chains for Electrical and Electronic Products, Promotors: Prof.dr.ir. J.A.E.E. van Nunen \& Prof.dr.ir. H.W.G.M. van Heck, EPS-2008152-LIS, ISBN: 978-90-5892-192-5, http://hdl.handle.net/1765/14785

Radkevitch, U.L, Online Reverse Auction for Procurement of Services, Promotor: Prof.dr.ir. H.W.G.M. van Heck, EPS-2008-137-LIS, ISBN: 978-90-5892-171-0, http://hdl.handle.net/1765/13497

Rinsum, M. van, Performance Measurement and Managerial Time Orientation, Promotor: Prof.dr. F.G.H. Hartmann, EPS-2006-088-F\&A, ISBN: 90-5892-121-2, http://hdl.handle.net/1765/7993

Roelofsen, E.M., The Role of Analyst Conference Calls in Capital Markets, Promotors: Prof.dr. G.M.H. Mertens \& Prof.dr. L.G. van der Tas RA, EPS-2010-190-F\&A, ISBN: 978-90-5892228-1, http://hdl.handle.net/1765/1

Romero Morales, D., Optimization Problems in Supply Chain Management, Promotors: Prof.dr.ir. J.A.E.E. van Nunen \& Dr. H.E. Romeijn, EPS-2000-003-LIS, ISBN: 90-9014078-6, http:/ / hdl.handle.net/1765/865

Roodbergen, K.J., Layout and Routing Methods for Warehouses, Promotors: Prof.dr. M.B.M. de Koster \& Prof.dr.ir. J.A.E.E. van Nunen, EPS-2001-004-LIS, ISBN: 90-5892-005-4, http:/ /hdl.handle.net/1765/861

Rook, L., Imitation in Creative Task Performance, Promotor: Prof.dr. D.L. van Knippenberg, EPS-2008-125-ORG, http://hdl.handle.net/1765/11555

Rosmalen, J. van, Segmentation and Dimension Reduction: Exploratory and Model-Based Approaches, Promotor: Prof.dr. P.J.F. Groenen, EPS-2009-165-MKT, ISBN: 978-90-5892-2014, http:/ /hdl.handle.net/1765/15536

Rus, D., The Dark Side of Leadership: Exploring the Psychology of Leader Self-serving Behavior, Promotor: Prof.dr. D.L. van Knippenberg, EPS-2009-178-ORG, http://hdl.handle.net/1765/1

Samii, R., Leveraging Logistics Partnerships: Lessons from Humanitarian Organizations, Promotors: Prof.dr.ir. J.A.E.E. van Nunen \& Prof.dr.ir. L.N. Van Wassenhove, EPS-2008153-LIS, ISBN: 978-90-5892-186-4, http://hdl.handle.net/1765/14519 
Schaik, D. van, MEA in Japan: An Analysis of Merger Waves and Hostile Takeovers,

Promotors: Prof.dr. J. Spronk \& Prof.dr. J.P.M. Groenewegen, EPS-2008-141-F\&A, ISBN: 978-90-5892-169-7, http:// hdl.handle.net/1765/13693

Schauten, M.B.J., Valuation, Capital Structure Decisions and the Cost of Capital, Promotors: Prof.dr. J. Spronk \& Prof.dr. D. van Dijk, EPS-2008-134-F\&A, ISBN: 978-90-5892-172-7, http://hdl.handle.net/1765/13480

Schramade, W.L.J., Corporate Bonds Issuers, Promotor: Prof.dr. A. De Jong, EPS-2006-092F\&A, ISBN: 90-5892-125-5, http://hdl.handle.net/1765/8191

Schweizer, T.S., An Individual Psychology of Novelty-Seeking, Creativity and Innovation, Promotor: Prof.dr. R.J.M. van Tulder, EPS-2004-048-ORG, ISBN: 90-5892-077-1, http://hdl.handle.net/1765/1818

Six, F.E., Trust and Trouble: Building Interpersonal Trust Within Organizations, Promotors: Prof.dr. B. Nooteboom \& Prof.dr. A.M. Sorge, EPS-2004-040-ORG, ISBN: 90-5892-064-X, http://hdl.handle.net/1765/1271

Slager, A.M.H., Banking across Borders, Promotors: Prof.dr. R.J.M. van Tulder \& Prof.dr. D.M.N. van Wensveen, EPS-2004-041-ORG, ISBN: 90-5892-066-6, http://hdl.handle.net/1765/1301

Sloot, L., Understanding Consumer Reactions to Assortment Unavailability, Promotors: Prof.dr. H.R. Commandeur, Prof.dr. E. Peelen \& Prof.dr. P.C. Verhoef, EPS-2006-074MKT, ISBN: 90-5892-102-6, http://hdl.handle.net/1765/7438

Smit, W., Market Information Sharing in Channel Relationships: Its Nature, Antecedents and Consequences, Promotors: Prof.dr.ir. G.H. van Bruggen \& Prof.dr.ir. B. Wierenga, EPS2006-076-MKT, ISBN: 90-5892-106-9, http:/ /hdl.handle.net/1765/7327

Sonnenberg, M., The Signalling Effect of HRM on Psychological Contracts of Employees: A Multi-level Perspective, Promotor: Prof.dr. J. Paauwe, EPS-2006-086-ORG, ISBN: 90-5892119-0, http:/ /hdl.handle.net/1765/7995

Sotgiu, F., Not All Promotions are Made Equal: From the Effects of a Price War to Cross-chain Cannibalization, Promotors: Prof.dr. M.G. Dekimpe \& Prof.dr.ir. B. Wierenga, EPS-2010203-MKT, ISBN: 978-90-5892-238-0, http://hdl.handle.net/1765/1 
Speklé, R.F., Beyond Generics: A closer Look at Hybrid and Hierarchical Governance, Promotor: Prof.dr. M.A. van Hoepen RA, EPS-2001-008-F\&A, ISBN: 90-5892-011-9, http:/ /hdl.handle.net/1765/357

Srour, F.J., Dissecting Drayage: An Examination of Structure, Information, and Control in Drayage Operations, Promotor: Prof.dr. S.L. van de Velde, EPS-2010-186-LIS, http://hdl.handle.net/1765/1

Stam, D.A., Managing Dreams and Ambitions: A Psychological Analysis of Vision Communication, Promotor: Prof.dr. D.L. van Knippenberg, EPS-2008-149-ORG, http://hdl.handle.net/1765/14080

Stienstra, M., Strategic Renewal in Regulatory Environments: How Inter-and Intraorganisational Institutional Forces Influence European Energy Incumbent Firms, Promotors: Prof.dr.ir. F.A.J. Van den Bosch \& Prof.dr. H.W. Volberda, EPS-2008-145-STR, ISBN: 97890-5892-184-0, http://hdl.handle.net/1765/13943

Sweldens, S.T.L.R., Evaluative Conditioning 2.0: Direct versus Associative Transfer of Affect to Brands, Promotor: Prof.dr. S.M.J. van Osselaer, EPS-2009-167-MKT, ISBN: 978-90-5892204-5, http://hdl.handle.net/1765/1

Szkudlarek, B.A., Spinning the Web of Reentry: [Re]connecting reentry training theory and practice, Promotor: Prof.dr. S.J. Magala, EPS-2008-143-ORG, ISBN: 978-90-5892-177-2, http:/ /hdl.handle.net/1765/13695

Tempelaar, M.P., Organizing for Ambidexterity: Studies on the Pursuit of Exploration and Exploitation through Differentiation, Integration, Contextual and Individual Attributes, Promotors: Prof.dr.ing. F.A.J. van den Bosch \& Prof.dr. H.W. Volberda, EPS-2010-191STR, ISBN: 978-90-5892-231-1, http://hdl.handle.net/1765/1

Teunter, L.H., Analysis of Sales Promotion Effects on Household Purchase Behavior, Promotors: Prof.dr.ir. B. Wierenga \& Prof.dr. T. Kloek, EPS-2002-016-MKT, ISBN: 90-5892-029-1, http://hdl.handle.net/1765/868

Tims, B., Empirical Studies on Exchange Rate Puzzles and Volatility, Promotor: Prof.dr. C.G. Koedijk, EPS-2006-089-F\&A, ISBN: 90-5892-113-1, http://hdl.handle.net/1765/8066 
Tiwari, V., Transition Process and Performance in IT Outsourcing: Evidence from a Field Study and Laboratory Experiments, Promotors: Prof.dr.ir. H.W.G.M. van Heck \& Prof.dr. P.H.M. Vervest, EPS-2010-201-LIS, ISBN: 978-90-5892-241-0, http://hdl.handle.net/1765/1

Tuk, M.A., Is Friendship Silent When Money Talks? How Consumers Respond to Word-ofMouth Marketing, Promotors: Prof.dr.ir. A. Smidts \& Prof.dr. D.H.J. Wigboldus, EPS-2008130-MKT, ISBN: 978-90-5892-164-2, http://hdl.handle.net/1765/12702

Valck, K. de, Virtual Communities of Consumption: Networks of Consumer Knowledge and Companionship, Promotors: Prof.dr.ir. G.H. van Bruggen \& Prof.dr.ir. B. Wierenga, EPS2005-050-MKT, ISBN: 90-5892-078-X, http://hdl.handle.net/1765/6663

Valk, W. van der, Buyer-Seller Interaction Patterns During Ongoing Service Exchange, Promotors: Prof.dr. J.Y.F. Wynstra \& Prof.dr.ir. B. Axelsson, EPS-2007-116-MKT, ISBN: 905892-146-8, http://hdl.handle.net/1765/10856

Verheijen, H.J.J., Vendor-Buyer Coordination in Supply Chains, Promotor: Prof.dr.ir. J.A.E.E. van Nunen, EPS-2010-194-LIS, ISBN: 90-5892-234-2, http://hdl.handle.net/1765/1

Verheul, I., Is There a (Fe)male Approach? Understanding Gender Differences in Entrepreneurship, Prof.dr. A.R. Thurik, EPS-2005-054-ORG, ISBN: 90-5892-080-1, http://hdl.handle.net/1765/2005

Verwijmeren, P., Empirical Essays on Debt, Equity, and Convertible Securities, Promotors: Prof.dr. A. de Jong \& Prof.dr. M.J.C.M. Verbeek, EPS-2009-154-F\&A, ISBN: 978-90-5892187-1, http:/ /hdl.handle.net/1765/14312

Vis, I.F.A., Planning and Control Concepts for Material Handling Systems, Promotors: Prof.dr. M.B.M. de Koster \& Prof.dr.ir. R. Dekker, EPS-2002-014-LIS, ISBN: 90-5892-021-6, http://hdl.handle.net/1765/866

Vlaar, P.W.L., Making Sense of Formalization in Interorganizational Relationships: Beyond Coordination and Control, Promotors: Prof.dr.ir. F.A.J. Van den Bosch \& Prof.dr. H.W. Volberda, EPS-2006-075-STR, ISBN 90-5892-103-4, http://hdl.handle.net/1765/7326

Vliet, P. van, Downside Risk and Empirical Asset Pricing, Promotor: Prof.dr. G.T. Post, EPS2004-049-F\&A, ISBN: 90-5892-07-55, http://hdl.handle.net/1765/1819 
Vlist, P. van der, Synchronizing the Retail Supply Chain, Promotors: Prof.dr.ir. J.A.E.E. van Nunen \& Prof.dr. A.G. de Kok, EPS-2007-110-LIS, ISBN: 90-5892-142-0,

http://hdl.handle.net/1765/10418

Vries-van Ketel E. de, How Assortment Variety Affects Assortment Attractiveness:

A Consumer Perspective, Promotors: Prof.dr. G.H. van Bruggen \& Prof.dr.ir. A. Smidts, EPS-2006-072-MKT, ISBN: 90-5892-101-8, http://hdl.handle.net/1765/7193

Vromans, M.J.C.M., Reliability of Railway Systems, Promotors: Prof.dr. L.G. Kroon, Prof.dr.ir. R. Dekker \& Prof.dr.ir. J.A.E.E. van Nunen, EPS-2005-062-LIS, ISBN: 90-5892089-5, http://hdl.handle.net/1765/6773

Vroomen, B.L.K., The Effects of the Internet, Recommendation Quality and Decision Strategies on Consumer Choice, Promotor: Prof.dr. Ph.H.B.F. Franses, EPS-2006-090-MKT, ISBN: 905892-122-0, http://hdl.handle.net/1765/8067

Waal, T. de, Processing of Erroneous and Unsafe Data, Promotor: Prof.dr.ir. R. Dekker, EPS2003-024-LIS, ISBN: 90-5892-045-3, http:/ / hdl.handle.net/1765/870

Waard, E.J. de, Engaging Environmental Turbulence: Organizational Determinants for Repetitive Quick and Adequate Responses, Promotors: Prof.dr. H.W. Volberda \& Prof.dr. J. Soeters, EPS-2010-189-STR, ISBN: 978-90-5892-229-8, http://hdl.handle.net/1765/1

Wall, R.S., Netscape: Cities and Global Corporate Networks, Promotor: Prof.dr. G.A. van der Knaap, EPS-2009-169-ORG, ISBN: 978-90-5892-207-6, http://hdl.handle.net/1765/1

Watkins Fassler, K., Macroeconomic Crisis and Firm Performance, Promotors: Prof.dr. J. Spronk \& Prof.dr. D.J. van Dijk, EPS-2007-103-F\&A, ISBN: 90-5892-138-3, http://hdl.handle.net/1765/10065

Weerdt, N.P. van der, Organizational Flexibility for Hypercompetitive Markets: Empirical Evidence of the Composition and Context Specificity of Dynamic Capabilities and Organization Design Parameters, Promotor: Prof.dr. H.W. Volberda, EPS-2009-173-STR, ISBN: 978-905892-215-1, http://hdl.handle.net/1765/1

Wennekers, A.R.M., Entrepreneurship at Country Level: Economic and Non-Economic Determinants, Promotor: Prof.dr. A.R. Thurik, EPS-2006-81-ORG, ISBN: 90-5892-115-8, http://hdl.handle.net/1765/7982 
Wielemaker, M.W., Managing Initiatives: A Synthesis of the Conditioning and KnowledgeCreating View, Promotors: Prof.dr. H.W. Volberda \& Prof.dr. C.W.F. Baden-Fuller, EPS2003-28-STR, ISBN: 90-5892-050-X, http://hdl.handle.net/1765/1042

Wijk, R.A.J.L. van, Organizing Knowledge in Internal Networks: A Multilevel Study, Promotor: Prof.dr.ir. F.A.J. van den Bosch, EPS-2003-021-STR, ISBN: 90-5892-039-9, http:/ /hdl.handle.net/1765/347

Wolters, M.J.J., The Business of Modularity and the Modularity of Business, Promotors: Prof. mr. dr. P.H.M. Vervest \& Prof.dr.ir. H.W.G.M. van Heck, EPS-2002-011-LIS, ISBN: 905892-020-8, http://hdl.handle.net/1765/920

Wubben, M.J.J., Social Functions of Emotions in Social Dilemmas, Promotors: Prof.dr. D. De Cremer \& Prof.dr. E. van Dijk, EPS-2009-187-ORG, http://hdl.handle.net/1765/1

Xu, Y., Empirical Essays on the Stock Returns, Risk Management, and Liquidity Creation of Banks, Promotor: Prof.dr. M.J.C.M. Verbeek, EPS-2010-188-F\&A, http://hdl.handle.net/1765/1

Yang, J., Towards the Restructuring and Co-ordination Mechanisms for the Architecture of Chinese Transport Logistics, Promotor: Prof.dr. H.E. Harlambides, EPS-2009-157-LIS, ISBN: 978-90-5892-198-7, http://hdl.handle.net/1765/14527

Yu, M., Enhancing Warehouse Perfromance by Efficient Order Picking, Promotor: Prof.dr. M.B.M. de Koster, EPS-2008-139-LIS, ISBN: 978-90-5892-167-3, http://hdl.handle.net/1765/13691

Zhang, X., Strategizing of Foreign Firms in China: An Institution-based Perspective, Promotor: Prof.dr. B. Krug, EPS-2007-114-ORG, ISBN: 90-5892-147-5, http://hdl.handle.net/1765/10721

Zhang, X., Scheduling with Time Lags, Promotor: Prof.dr. S.L. van de Velde, EPS-2010-206LIS,

ISBN: 978-90-5892-244-1, http://hdl.handle.net/1765/1

Zhu, Z., Essays on China's Tax System, Promotors: Prof.dr. B. Krug \& Prof.dr. G.W.J. Hendrikse, EPS-2007-112-ORG, ISBN: 90-5892-144-4, http://hdl.handle.net/1765/10502 
Zwart, G.J. de, Empirical Studies on Financial Markets: Private Equity, Corporate Bonds and Emerging Markets, Promotors: Prof.dr. M.J.C.M. Verbeek \& Prof.dr. D.J.C. van Dijk, EPS2008-131-F\&A, ISBN: 978-90-5892-163-5, http://hdl.handle.net/1765/12703 


\section{LANGUAGE ABSTRACTION IN WORD OF MOUTH}

In word of mouth, consumers talk about their experiences with products and services with other consumers. These conversations are important sources of information for consumers. While word of mouth has fascinated researchers and practitioners for many years, little attention has been paid to the question of how consumers talk about products and brands, and whether and how this moderates the extent to which they influence other consumers. This dissertation fills this gap, and focuses on the language that consumers use when they describe their experiences. For example, if your brand new shirt lost its color after one or two washes, you could say to your friend "My shirt has faded," or you could say "My shirt was of poor quality". In the former case, you provide a very concrete description of what has happened. In the latter, you use more abstract wording, which generalizes this single experience to an overall impression of the shirt's quality. This dissertation focuses on language abstraction, because abstractness is an important aspect of language, and it can be coded unambiguously and relatively easily. A series of experiments examined when and why consumers use abstract versus concrete language in word of mouth, and how these differences in language use affect the receiver of the word-of-mouth message. Among other things, the results show that consumers use more abstract language when they describe expriences that are in line with their prior opinions about a product, and that more abstract descriptions generally have a larger impact on other consumers.

\section{ERIM}

The Erasmus Research Institute of Management (ERIM) is the Research School (Onderzoekschool) in the field of management of the Erasmus University Rotterdam. The founding participants of ERIM are Rotterdam School of Management (RSM), and the Erasmus School of Economics (ESE). ERIM was founded in 1999 and is officially accredited by the Royal Netherlands Academy of Arts and Sciences (KNAW). The research undertaken by ERIM is focused on the management of the firm in its environment, its intra- and interfirm relations, and its business processes in their interdependent connections.

The objective of ERIM is to carry out first rate research in management, and to offer an advanced doctoral programme in Research in Management. Within ERIM, over three hundred senior researchers and PhD candidates are active in the different research programmes. From a variety of academic backgrounds and expertises, the ERIM community is united in striving for excellence and working at the forefront of creating new business knowledge.

Erasmus Research Institute of Management - ERIM Rotterdam School of Management (RSM) Erasmus School of Economics (ESE) P.O. Box 1738, 3000 DR Rotterdam The Netherlands
Tel.

info@erim.eur.n

Internet www.erim.eur.nl 